\title{
7 Konstruktikographische Generalisierungen
}

\author{
One might hope for a semantic theory to relate \\ expression meaning to utterance context. \\ However, [...] we should tackle the opposite \\ problem: how meaning construction processes \\ that generate utterance meanings might also \\ explain why we think of words, phrases, and \\ sentences as having meaning that is \\ independent of any particular context.
}

(Coulson 2001: 8)

Die Analysen, die ich insbesondere in Kapitel 6 vorgestellt habe, liefern über weite Strecken Ergebnisse auf Token-Ebene, da sie die Konstitution von KonstruktFrames etwa durch die Frage nach den durch KtE des KE EREIGNIS evozierten lexikalischen Frames und die semantische Motivierung von KtE und KEE durch FE eines lexikalischen Frames und des Konstruktions-Frames aufzeigen. Für eine konstruktikographische Beschreibung der drei untersuchten Konstruktionen müssen diese Ergebnisse auf Token-Ebene allerdings hinreichend generalisiert werden, um sie zu konstruktikographisch verwertbaren Informationen auf TypeEbene zu überführen. Der Begriff der Generalisierung ist für die Kognitive Linguistik von äußerster Wichtigkeit. Er findet sich bereits in einer der beiden von Lakoff (1990: 40, 1991: 53) formulierten commitments wieder. Das Gebot der Generalisierung, das durch eines dieser commitments repräsentiert wird, bildet das Ziel des linguistischen Beschreibungsinteresses ab, wenn es darin besteht, „to characterizing the general principles governing all aspects of human language." (Lakoff 1990: 40). ${ }^{1}$ Konstruktikographisch lässt sich dieses Ziel in ein konkretes Format fassen. Analysen auf Token-Ebene müssen in für eine Konstruktion konstruktikographisch verwertbare Informationen überführt werden, kurzum: in einen Konstruktionseintrag. Wie also lassen sich die bisher für die drei untersuchten Konstruktionen erzielten Ergebnisse auf eine solche Type-Ebene generalisieren und als konstruktikographisch relevante Eigenschaften einer Konstruktion angeben?

Um diese Frage zu beantworten, möchte ich zunächst einige Schlussfolgerungen aus den Ergebnissen aus Kapitel 5 und 6 ziehen. Die Analysen der variierenden semantischen Motivierung von KtE und des KEE der reflexiven Bewegungskonstruktion und ihrer verwandten Konstruktionen - insbesondere der reflexi-

1 Der Begriff der Generalisierung wird hingegen von Goldberg (2002: 327-330) vor allem gegen generativistische Konzepte wie Transformation und Derivation auf syntaktischer Ebene positioniert und ist demnach potenziell eingeschränkter als derjenige von Lakoff. 
ven Partikelverbkonstruktion - haben gezeigt, dass der Anteil des KonstruktionsFrames an Konstrukt-Frames stark von der semantischen Motivierung der einzelnen KtE und KEE abhängt. Kein Anteil des Konstruktions-Frames liegt vor, wenn alle KtE und ggf. das KEE durch FE des lexikalischen Frames motiviert werden (Unterabschnitt 6.2.1). Am größten ist der Anteil des Konstruktions-Frames, wenn ein KtE und das KEE ausschließlich durch diesen einfach motiviert werden, während die anderen KtE durch den lexikalische Frame motiviert werden (Variante c einer Frame-Anpassung, vgl. Unterabschnitt 4.4.1). In Anlehnung an das Postulat des Lexikon-Grammatik-Kontinuums von Langacker (1987: 3) könnte man diese beiden Extrempunkte sowie alle Fälle, die zwischen ihnen liegen, als ein Kontinuum der semantischen Motivierung eines Konstrukts verstehen. In diesem Sinne spricht Langacker (2005a: 152) von einem ,continuous spectrum in terms of how familiar it is for the verb to occur in the construction and how well the verb's meaning fits the constructional meaning. “ Dieses Kontinuum der semantischen Motivierung eines Konstrukts ist entscheidend unter anderem für die Messung semantischer Parameter, vor allem des Koerzionspotenzials (Abschnitte 5.5 und 7.4).

Die Tatsache, dass bestimmte Strukturelemente einer Konstruktion in den meisten Fällen, wie es die unterschiedlichen Konstruktikographie-Projekte praktizieren (vgl. Unterabschnitt 2.3.2), semantisch definiert werden, zeigt, dass es nicht ausreichend ist, eine Konstruktion nur schlicht aufgrund einer formalen Konstellation von KE, KEE und KorE zu beschreiben. ${ }^{2}$ So ist etwa eine semantische Definition von KE, KEE und KorE nötig, um strukturell analoge sprachliche Ausdrücke, die aber keine Konstrukte einer gegebenen Konstruktion sind, aus der Analyse auszuschließen (dazu Unterabschnitt 8.4.2). Am wichtigsten ist eine semantische Beschreibung einer Konstruktion auf Type-Ebene aber für die Konstruktikographie selbst, denn sie bildet den Ausgangspunkt für einen Konstruktionseintrag.

Die semantischen Eigenschaften der Strukturelemente wiederum ergeben sich in erster Linie aus der Konstitution von Konstrukt-Frames, also aus der frequenten semantischen Motivierung etwa von KtE und KEE durch FE lexikalischer Frames und des Konstruktions-Frames. Die Relevanz von FE für die Beschreibung von KE ist in der Konstruktikographie indes nicht unerkannt geblieben: Die wayKonstruktion, wie sie im FrameNet-Konstruktikon beschrieben wird, ist hierfür ein gutes Beispiel, denn wie schon in Unterabschnitt 2.3.2 gesehen, werden ihre KE in erster Linie nach FE des Frames Motion benannt und definiert. Diese Beobachtung ist Ausgangspunkt für die detaillierten Analysen der Strukturparallelen zwischen Konstruktionen und Frames, die ich in Kapitel 6 für die reflexive

2 Vgl. dazu schon Brugman (1996: 45): ,[T] he morphosyntactic properties which distinguish constructions are not sufficient to identify their constructional semantics“. 
Bewegungskonstruktion und die reflexive Partikelverbkonstruktion vorgestellt habe.

Eine wesentliche Erkenntnis dieser Analysen ist die Varianz in der Konstitution von Konstrukt-Frames. Je nach semantischer Motivierung der KtE und des KEE stellt sich die Konstellation instanziierter FE in einem Konstrukt, also auf Token-Ebene, sehr vielfältig dar. Man könnte nun annehmen, dass diese Varianz ein Problem darstellt, da Generalisierungen vor diesem Hintergrund schwierig erscheinen können. Aus gebrauchsbasierter Perspektive ist eine solche Varianz aber alles andere als problematisch, da sie den Blick auf Prozesse der Abstraktion und Generalisierung gerade erst ermöglicht, wie Bybee (2010) betont:

\begin{abstract}
The existence of gradience and variation does not negate the regular patterning within languages or the patterning across languages. However, it is important not to view the regularities as primary and the gradience and variation as secondary; rather the same factors operate to produce both regular patterns and the deviations. If language were a fixed mental structure, it would perhaps have discrete categories; but since it is a mental structure that is in constant use and filtered through processing activities that change it, there is variation and gradation. (Bybee 2010: 6)
\end{abstract}

In diesem Kapitel möchte ich nun einige methodologische und methodische Zugänge aufzeigen, wie die in den Kapiteln 5 und 6 zum Teil bereits geleisteten Analysen auf Token-Ebene über ihre Varianz hinweg zu konstruktikographisch verwertbaren Informationen generalisiert werden können. Im Zuge dessen präsentiere ich für die drei untersuchten Konstruktionen alle relevanten Ergebnisse dieser Generalisierung. In Abschnitt 7.1 kläre ich, welche Informationen eine konstruktikographische Beschreibung, konkret: ein Konstruktionseintrag, überhaupt enthalten muss. Dazu gehört die Frage, wie die in Kapitel 5 vorgestellten semantischen Parameter von Konstruktionen Eingang in einen Konstruktionseintrag finden können. In Abschnitt 7.2 beginne ich mit der konstruktikographischen Erfassung zunächst einiger allgemeiner Informationen, zu denen insbesondere die Namen der Konstruktionen (deren Wahl ich an dieser Stelle begründe) sowie die Differenzierung konstruktioneller Polysemie gehört. Abschnitt 7.3 widmet sich der Benennung und Definition der Strukturelemente der Konstruktionen, die einen wesentlichen Teil von deren konstruktikographischer Beschreibung ausmachen und auf semantische Parameter wie Präferenzen und Beschränkungen (für lexikalische Frames, vgl. Unterabschnitt 5.3.3) und Frame-Nähe (Abschnitt 5.4) zurückgreifen. Die drei darauffolgenden Abschnitte 7.4, 7.5 und 7.6 widmen sich schließlich dezidiert drei semantischen Parametern aus Kapitel 5, die als eigene Datenpunkte in einen Konstruktionseintrag eingehen müssen: dem Koerzionspotenzial, der Produktivität sowie der emergenten Struktur. Für sie geht es nicht nur darum, Methoden zu ihrer Messung zu entwickeln, sondern gleichsam ihre ,Werte‘ zu be- 
stimmen, um diese in einen Konstruktionseintrag überführen zu können. Wie die Konstruktionseinträge für die drei untersuchten Konstruktionen konkret aussehen können, ist im dreiteiligen Zusatzmaterial zu sehen. ${ }^{3}$

\subsection{Konstruktikographische Beschreibung von Konstruktionen}

Nachdem ich in Kapitel 4 die Begriffe von Konstruktions-Frame und Konstruktionsbedeutung in ein konstruktionssemantisches Modell eingebettet und in Kapitel 5 sieben semantische Parameter von Konstruktionen vorgestellt habe, die die semantischen Eigenschaften einer Konstruktion näher bestimmen und messbar machen sollen, stellt sich nun die Frage, in welcher Form diese Informationen in die konstruktikographische Beschreibung einer Konstruktion, also in einen Konstruktionseintrag, eingehen müssen. Deshalb möchte ich zu Beginn zunächst auf die Bestandteile der konstruktikographischen Beschreibung einer Konstruktion eingehen, die der Endpunkt jeder Analyse sind. Damit soll einerseits deutlich gemacht werden, welche Relevanz etwa die Daten haben, die ich in Kapitel 6 hinsichtlich der Strukturparallelen von Konstruktionen und Frames erhoben habe, andererseits geht es aber auch um basalere Aspekte wie die Namen der Konstruktionen, deren Begründung ich bisher noch nicht diskutiert habe. Wie die Informationen, die Eingang in einen Konstruktionseintrag finden müssen, konkret zustande kommen, wird später Gegenstand der Abschnitte 7.3 bis 7.6 sein.

In diesem Abschnitt möchte ich zunächst auf Voraussetzungen für die konstruktikographische Beschreibung von Konstruktionen eingehen. Hierzu gehört eine wesentliche Frage, die sich bei der Zusammenstellung von Informationen für einen Konstruktionseintrag ergibt, nämlich welche Inhalte ein Konstruktionseintrag überhaupt enthalten muss, und um die es in Unterabschnitt 7.1.1 gehen soll. Im Anschluss möchte ich in Unterabschnitt 7.1.2 die Frage beantworten, wie das in Kapitel 4 eingeführte konstruktionssemantische Modell Eingang in einen solchen Konstruktionseintrag finden kann. Damit hängen die in Kapitel 5 skizzierten semantischen Parameter von Konstruktionen eng zusammen. Auch für sie muss geklärt werden, in welcher Form sie Eingang in einen Konstruktionseintrag finden und an welchen Stellen sich die durch sie ermittelbaren Informationen in einem Konstruktionseintrag wiederfinden müssen. Darauf gehe ich in Unterabschnitt 7.1.3 ein.

3 Das Zusatzmaterial mit den drei Konstruktionseinträgen kann unter https://www.degruyter. com/document/isbn/9783110762341/html heruntergeladen werden. 


\subsubsection{Inhalte eines Konstruktionseintrags}

Ziel jeder konstruktikographischen Analyse ist ein Konstruktionseintrag. Der Frage, wie die Ergebnisse einer solchen Analyse dokumentiert werden müssen, muss die allgemeinere Frage vorangestellt werden, welche Bestandteile ein Konstruktionseintrag enthalten muss. ${ }^{4}$ In der konstruktikographischen Forschung existiert bisher kein Standard für obligatorische Inhalte eines Konstruktionseintrags, was nicht unwesentlich mit den unterschiedlichen Zielsetzungen der einzelnen konstruktikographischen Projekte (dazu Abschnitt 2.3) zusammenhängen dürfte.

Hinsichtlich der Inhalte von Konstruktionseinträgen, die ein Konstruktikon konstituieren, ist es jedenfalls offensichtlich, „[d]ass Umfang und Beschreibungstiefe eines [...] Referenzkonstruktikons, [und somit auch eines Konstruktionseintrags, A.W.] wie bei traditionellen Wörterbüchern auch, von den Zielsetzungen bezüglich der Funktionen, die ein solches Konstruktikon erfüllen soll, und den intendierten Benutzergruppen abhängt“ (Herbst 2016: 172). So macht es für die Inhalte eines Konstruktionseintrags einen erheblichen Unterschied, ob ein Konstruktikon für Fremdsprachenlernende oder als fachwissenschaftliche Ressource konzipiert ist (vgl. auch Herbst 2019: 7-9). Bisherige Vorschläge scheinen eher Letzteres im Blick zu haben, ${ }^{5}$ weshalb ich mich für die konstruktikographische Generalisierung der drei untersuchten Konstruktionen daran orientiere. Trotz dieser (impliziten) gemeinsamen Ausrichtung existierender Vorschläge für die Inhalte eines Konstruktionseintrags zeigt sich, dass ihre Breite durchaus unterschiedlich ausfällt. So stellen Ziem \& Flick (2018: Abschn. 3.4) einen exemplarischen Konstruktionseintrag auf, von dem aus mögliche obligatorische Inhalte abzuleiten sind. Eine dezidierte und recht umfassende Auflistung möglicher Inhalte bieten Boas \& Ziem (2018b: 202). Ziem \& Flick (2019: 208) sowie Ziem, Flick \& Sandkühler (2019: 80) stellen zwei exakt identische Listen mit Inhalten eines Konstruktionseintrags vor. Tabelle 7.1 stellt diese vier Vorschläge einander gegenüber, wobei Überschneidungen durch Alignierung in einer Zeile dargestellt sind. Die originäre Reihenfolge in den einzelnen Listen ist deshalb nicht beibehalten.

4 Noch allgemeiner ist die Frage, welche Inhalte ein Konstruktikon enthalten muss und nach welchen Maßstäben diese Inhalte ausgewählt werden müssen. Vgl. Herbst (2016, 2019: 7-9) sowie Lyngfelt (2018: 13-14) und Boas, Lyngfelt \& Torrent (2019: 40-48) für einige Vorschläge.

5 Lyngfelt (2018: 14) bemerkt, dass die meisten konstruktikographischen Projekte grundsätzlich nicht auf einen einzelnen Anwendungszweck beschränkt sind, betont aber zugleich, dass zumindest das Schwedische Konstruktikon und das Russische Konstruktikon Schwerpunkte auf die Anwendung durch Fremdsprachenlernende legen. Bei den im Folgenden zu diskutierenden Vorschlägen für die Inhalte eines Konstruktionseintrags werden diese Unterschiede nicht reflektiert. 


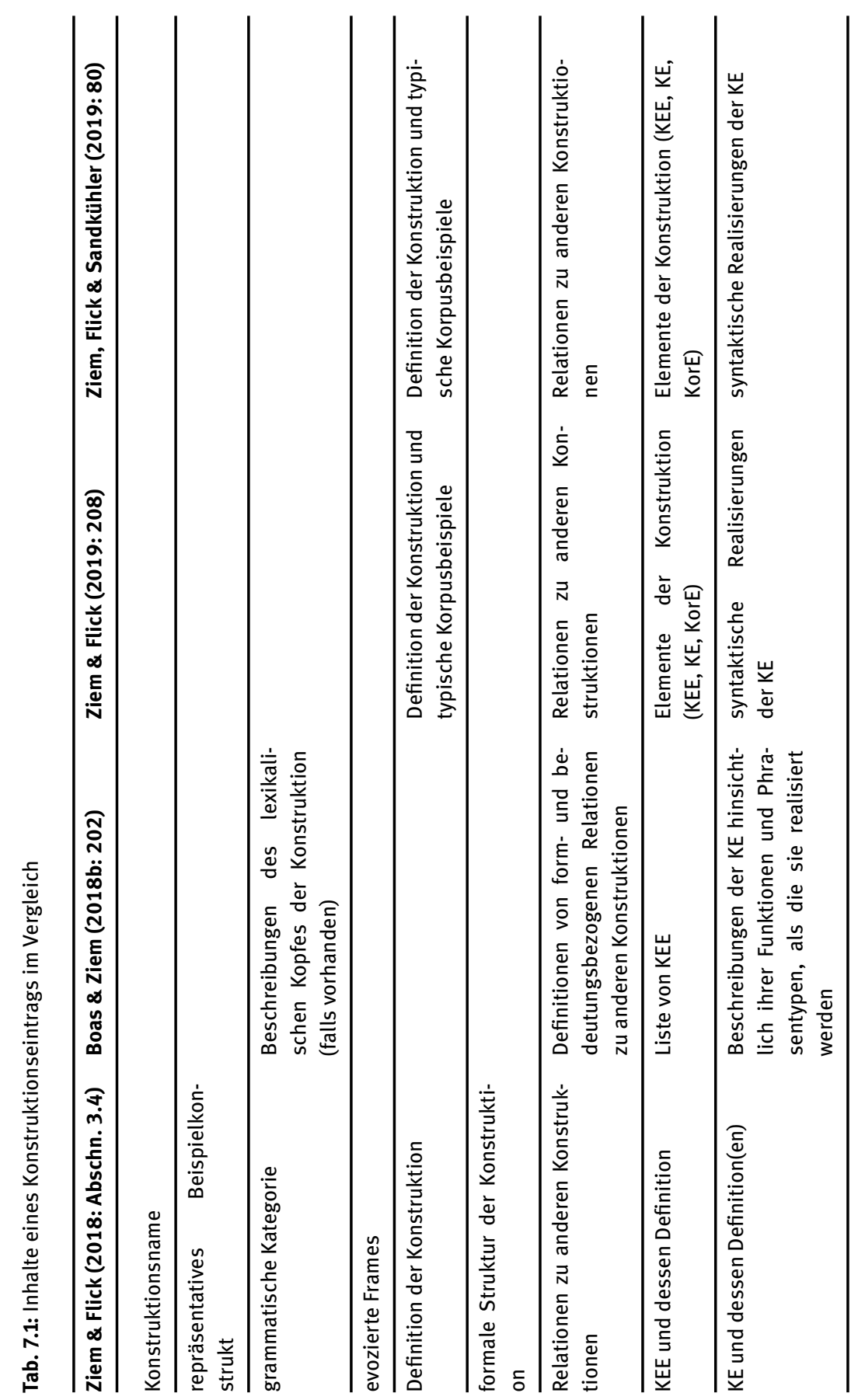




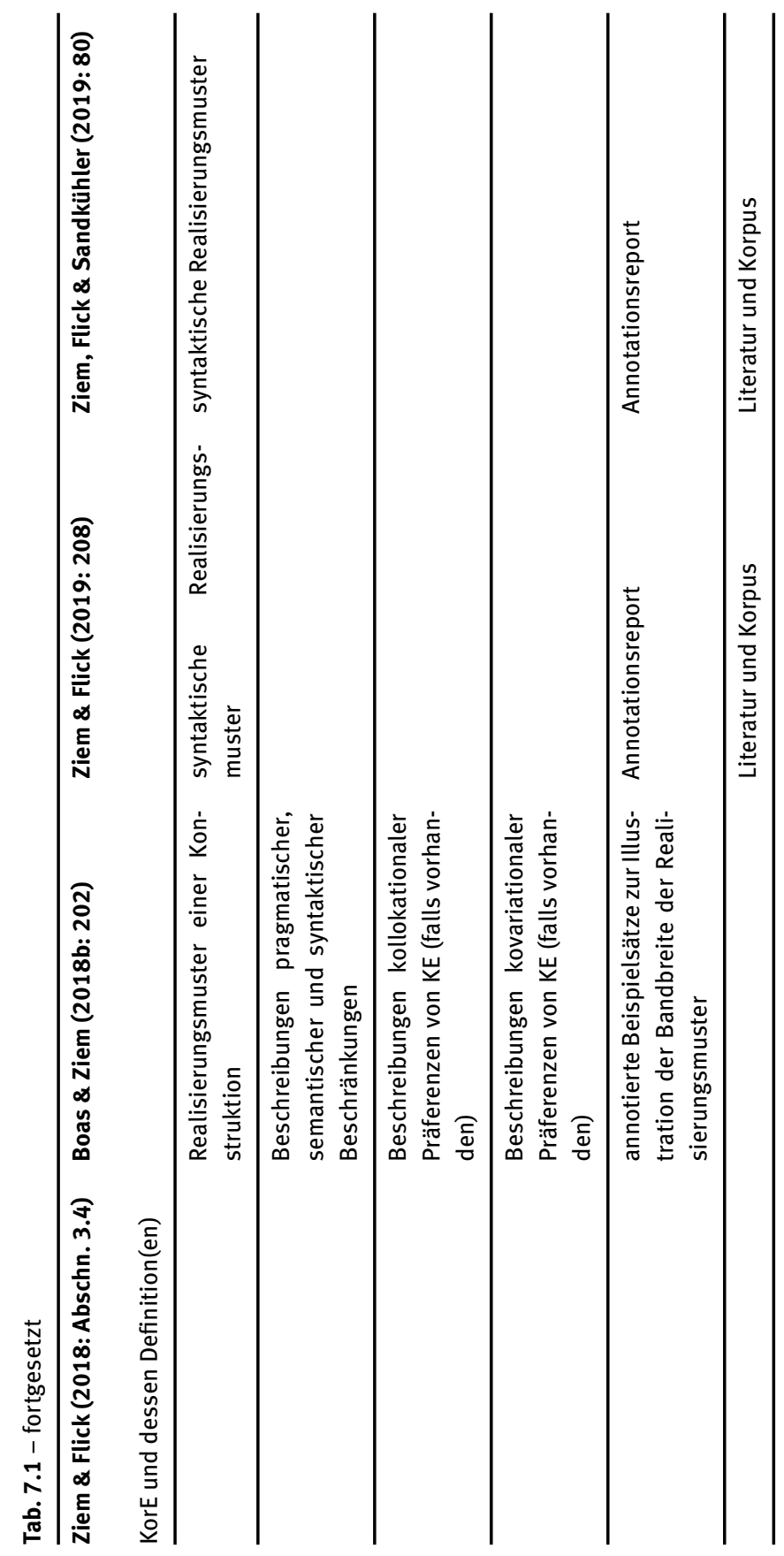


Ergänzend zu diesem sich in der Forschungsliteratur langsam abzeichnenden Kanon an obligatorischen Inhalten eines Konstruktionseintrags müssen freilich einige der im Rahmen des konstruktionssemantischen Modells aus Kapitel 4 zu reformulierende Inhalte (etwa die Angabe des Konstruktions-Frames betreffend) berücksichtigt sowie insbesondere die semantischen Parameter von Konstruktionen (Kapitel 5) einbezogen werden. Bevor ich in Unterabschnitt 7.1.3 diskutiere, wie sich die semantischen Parameter von Konstruktionen in einen Konstruktionseintrag einfügen lassen (worauf die Vorschläge in Tabelle 7.1 bereits teilweise vorbereitet sind), möchte ich aus den vier unterschiedlichen Konzeptionen zunächst eine Synthese bilden.

Aus konstruktionssemantischer Perspektive fällt hinsichtlich des unterschiedlichen Umfangs der vier Listen auf, dass einzig Ziem \& Flick (2018: Abschn. 3.4) auf einen durch eine Konstruktion evozierten Frame hinweisen, gewissermaßen also einen Konstruktions-Frame im Sinne des konstruktionssemantischen Modells (Kapitel 4). Ich komme darauf in Unterabschnitt 7.1.2 zurück. Außerdem sind Ziem \& Flick (2018: Abschn. 3.4) die einzigen, die dezidiert den Konstruktionsnamen mit in ihre Liste aufnehmen, wobei die anderen drei Konzeptionen diesen vermutlich implizit berücksichtigen, ohne ihn eigens in ihre Listen aufzunehmen.

Eine vorläufige Synthese der vier in Tabelle 7.1 gegenübergestellten Konzeptionen eines Konstruktionseintrag kann aus konstruktionssemantischer Sicht wie in der folgenden Auflistung aussehen, wobei dort noch nicht explizit die semantischen Parameter von Konstruktionen berücksichtigt sind. ${ }^{6}$ Deshalb werde ich diese Liste in Unterabschnitt 7.1.3 noch hinsichtlich der semantischen Parameter erweitern. Die nunmehr folgende Liste ist freilich vorrangig auf die drei untersuchten Konstruktionen zugeschnitten und kann für Konstruktionen anderer Art entsprechend anders aussehen. Die Liste ist also nicht als allgemeingültige Struktur eines Konstruktionseintrags zu verstehen, sondern soll in erster Linie die formalen und semantischen Eigenschaften der drei untersuchten Konstruktionen erfassen. Es ist ohnehin selbstverständlich, dass Art und Menge der Informationen, die in einem Konstruktionseintrag enthalten sein müssen, von der Art der Konstruk-

\footnotetext{
6 Ausgeklammert lasse ich hier einen Annotationsreport sowie Angaben zu relevanter Forschungsliteratur und den zugrunde liegenden Korpora, wie sie in den Auflistungen von Ziem \& Flick (2019: 208) sowie Ziem, Flick \& Sandkühler (2019: 80) erscheinen. Da die letzten beiden Punkte ohnehin Bestandteil der vorliegenden Arbeit sind, dupliziere ich sie in den Konstruktionseinträgen im Zusatzmaterial nicht noch einmal. Auf einen vollständigen Annotationsreport verzichte ich aus Platzgründen, da die Bandbreite der annotierten Daten bereits ausführlich gezeigt wurde und jener somit einer Wiederholung größtenteils bereits zitierter Daten entspräche. In einer digitalen Ressource in Form einer Datenbank besteht dieses Problem freilich nicht.
} 
tion abhängen, weshalb für andere als die hier untersuchten Konstruktionen potenziell bestimmte Aspekte ein anderes Gewicht bekommen können oder weitere hinzugenommen werden müssen. ${ }^{7}$

Jeder nummerierte Punkt in der folgenden Liste entspricht einem Datenpunkt eines Konstruktionseintrags, also einem Informationsabschnitt, der bisweilen durch weitere Unteraspekte binnendifferenziert werden kann. Um deutlich zu machen, was unter den einzelnen Datenpunkten zusammengefasst wird, füge ich ihnen jeweils eine kurze Beschreibung an.

1. Konstruktionsname: Die Bezeichnung der Konstruktion, die bereits im Titel des Konstruktionseintrags enthalten ist.

2. Repräsentatives Beispielkonstrukt: Ein ausgewählter Beleg, der ein Konstrukt enthält, das zur schnellen Illustration der Konstruktion dient.

3. Schematische Angabe der formalen Struktur: Eine Darstellung der formalen Struktur der Konstruktion, die sich aus den folgenden beiden Komponenten zusammensetzt.

- Beteiligte Phrasentypen: Die Phrasentypen, als die die KtE der einzelnen KE sowie KEE und KorE realisiert werden.

- Lexikalisch fixierte Elemente: Die KEE und KorE der Konstruktion, wobei sie nicht zwingend als invariante LE angegeben werden müssen, sondern auch Kategorien umfassen können, etwa Reflexiva bei allen drei untersuchten Konstruktionen, Verbpartikeln bei der reflexiven Partikelverbkonstruktion oder eine Bandbreite von Artikeln und Nomen bei der reflexiven Weg-Konstruktion.

4. Konstruktions-Frame: Der Frame, der als Konstruktions-Frame der Konstruktion gelten kann, im Falle der drei untersuchten Konstruktionen also Motion.

5. Definition der Konstruktion: Eine kurze allgemeine Charakterisierung der Konstruktion, ihrer formalen und semantischen Eigenschaften.

6. KE und deren Definitionen: Eine Liste der KE der Konstruktion inklusive ihrer formalen und semantischen Eigenschaften, die die folgenden Komponenten umfassen.

- Name des KE: Die Bezeichnung des KE.

- Definition des KE: Eine kurze Definition des KE.

7 Ein Beispiel ist die Kategorie des KorE (zur Terminologie Unterabschnitt 2.3.1), das unter den drei untersuchten Konstruktionen nur für die reflexive Weg-Konstruktion relevant ist. Da dies ein trivialer Fall ist, muss die Auswahl der Bestandteile eines Konstruktionseintrag hinsichtlich dieses Punktes - anders als bei einigen Punkten von Boas \& Ziem (2018b: 202), siehe unten nicht weiter begründet werden. 
- Formale Realisierung des KE: Angaben zur formalen Realisierung des KE, bei Argumentstruktur-Konstruktionen wie den drei untersuchten insbesondere in Form von Phrasentypen.

- Annotierte Beispielbelege: $\mathrm{Zu}$ jedem KE sollte mindestens ein allein auf dieses KE hin annotierter Beispielbeleg zur Illustration gehören.

7. KEE und deren Definition(en): Die Liste des bzw. der KEE und eine kurze Beschreibung seiner/ihrer formalen und semantischen Eigenschaften.

8. KorE und deren Definition(en): Die Liste des bzw. der KorE und eine kurze Beschreibung seiner/ihrer formalen und semantischen Eigenschaften.

9. Relationen zu anderen Konstruktionen: Relationen zu anderen Konstruktionen im Konstruktikon. Diese können anstelle von strikten Vererbungsrelationen auch als Familienähnlichkeiten (vgl. Unterabschnitt 3.2.3) beschrieben werden.

- Formale Relationen: Formale Relationen bzw. Familienähnlichkeiten zu anderen Konstruktionen.

- Semantische Relationen: Semantische Relationen bzw. Familienähnlichkeiten zu anderen Konstruktionen.

Obwohl diese Liste bereits auf die drei untersuchten Konstruktionen zugeschnitten ist, erscheint es nötig, den Ausschluss einiger Inhalte, die ich aus den Vorschlägen in Tabelle 7.1 nicht übernommen habe, zu kommentieren.

- Auf die Dokumentation von Realisierungsmustern (vierter und achter Punkt bei Boas \& Ziem 2018b: 202) verzichte ich, da die semantische Motivierung der KtE durch FE des lexikalischen Frames und des Konstruktions-Frames für die drei untersuchten Konstruktionen im Vordergrund steht und nicht mögliche syntagmatische Abfolgen von als KtE instanziierten KE sowie KEE und KorE. Wie bereits die Feststellung von Präferenzen für lexikalische Frames (Unterabschnitt 5.3.3) liefert die Untersuchung semantischer Motivierungen inhärente semantische Aussagen, sodass formale Realisierungsmuster (ohne eine Interpretation, die noch zu leisten wäre) lediglich einen sekundären Informationswert besitzen. Dass sie jedoch nicht gänzlich irrelevant sind, haben die Analysen der reflexiven Partikelverbkonstruktion in Unterabschnitt 6.4.2 gezeigt.

- Semantische Beschränkungen (fünfter Punkt bei Boas \& Ziem 2018b: 202) habe ich, wie bereits in Unterabschnitt 5.3.1 argumentiert, innerhalb des semantischen Parameters der Beschränkungen und Präferenzen subsumiert. Da hierbei insbesondere Präferenzen für lexikalische Frames einen übergeordneten Parameter darstellen (Unterabschnitt 5.3.3), erscheint dieser nicht gesondert unter den semantischen Parametern von Konstruktionen, sondern liefert die Datengrundlage für die Analysen der Frame-Nähe, des Koerzions- 
potenzials, der Produktivität und letztlich auch der emergenten Struktur. Syntaktische Beschränkungen, die ich in Unterabschnitt 5.3.2 untersucht habe, sind zu großen Teilen in den Definitionen der Strukturelemente enthalten und erhalten keinen eigenen Datenpunkt. Pragmatische Beschränkungen sind bei den drei untersuchten Konstruktionen vor dem Hintergrund des hier entwickelten konstruktionssemantischen Ansatzes zu vernachlässigen.

- Kollokationelle und Kovariationelle Präferenzen (sechster und siebter Punkt bei Boas \& Ziem 2018b: 202) spiegeln sich bereits in der Analyse der emergenten Struktur wider (als Variante b in Unterabschnitt 5.7.2), weshalb sie unter diesem semantischen Parameter subsumiert werden.

Wie bereits erwähnt, fehlt in der oben zusammengestellten Liste noch die explizite Berücksichtigung des konstruktionssemantischen Modells sowie der semantischen Parameter von Konstruktionen.

\subsubsection{Das konstruktionssemantische Modell in einem Konstruktionseintrag}

Aus den Vorschlägen für die möglichen Inhalte eines Konstruktionseintrags in Tabelle 7.1 in Unterabschnitt 7.1.1 lässt sich eine bisher geringe Beachtung genuin frame-semantischer Aspekte ableiten. Wie in Unterabschnitt 7.1.1 erwähnt, ist der exemplarische Konstruktionseintrag von Ziem \& Flick (2018: Abschn. 3.4) der einzige der vier Vorschläge, der den von einer Konstruktion evozierten Frame, gewissermaßen also den Konstruktions-Frame, berücksichtigt. Dies wirft die Frage auf, wie das konstruktionssemantische Modell (Kapitel 4) ebenso wie die semantischen Parameter von Konstruktionen (Kapitel 5) in einem Konstruktionseintrag verarbeitet werden können.

Die Frage nach der Relevanz des konstruktionssemantischem Modells für die Zusammenstellung eines Konstruktionseintrags betrifft die drei Frame- und Bedeutungstypen selbst: Inwiefern müssen lexikalische Frames und lexikalische Bedeutungen, Konstruktions-Frame und Konstruktionsbedeutung sowie KonstruktFrames und Konstruktbedeutungen Erwähnung in einem Konstruktionseintrag finden? Ich gehe dazu die drei Frame- und Bedeutungtypen in dieser Reihenfolge durch.

Wie schon in Unterabschnitt 7.1.1 angedeutet und in die vorläufige Liste der Inhalte eines Konstruktionseintrags aufgenommen, ist es $\mathrm{zu}$ allererst der Konstruktions-Frame, der in einem Konstruktionseintrag dokumentiert werden muss. Um einen Konstruktions-Frame von lexikalischen Frames abzugrenzen, habe ich in Abschnitt 4.3 das Kriterium der Invarianz eingeführt: KonstruktionsFrames sind über alle Konstrukte (einer Lesart) einer Konstruktion hinweg inva- 
riant, so liegt eben allen Konstrukten der drei hier untersuchten Konstruktionen Motion als Konstruktions-Frame zugrunde. Da ein Konstruktions-Frame über alle Konstrukte der Konstruktion hinweg invariant ist, kann er problemlos auf der Ebene des Konstruktionseintrags dokumentiert werden. Darüber hinaus ist der Konstruktions-Frame aufgrund seiner Fähigkeit zur Differenzierung der Lesarten einer polysemen Konstruktion (Unterabschnitt 5.2.1) für einen Konstruktionseintrag besonders relevant, wenn für eine polyseme Konstruktion unterschiedliche Konstruktionseinträge angenommen werden müssen. In diesem Fall ist der Konstruktions-Frame von derartiger Prominenz, dass er potenziell bereits im Konstruktionsnamen und damit dem Titel des Konstruktionseintrags erscheinen kann (dazu Unterabschnitt 7.2.1). Gleiches gilt analog für eine gegenüber dem Konstruktions-Frame durch Standardwerte spezifizierte Konstruktionsbedeutung.

Lexikalische Frames und lexikalische Bedeutungen finden an unterschiedlichen Stellen in einen Konstruktionseintrag Eingang. Sie unterscheiden sich von einem Konstruktions-Frame und einer Konstruktionsbedeutung durch ihre Varianz über die Konstrukte einer Konstruktion hinweg (vgl. Unterabschnitt 4.2.3) und sind deshalb anders als der Konstruktions-Frame zu behandeln. Sie sind nicht direkt auf Type-Ebene in einem Konstruktionseintrag festzuhalten und können somit selbst nicht wie der Konstruktions-Frame Teil einer konstruktikographischen Beschreibung sein. Dennoch sind lexikalische Frames und lexikalische Bedeutungen für die konstruktikographische Beschreibung relevant, da sie eine wichtige Grundlage für die Benennung und Definition von KE und KEE (Abschnitt 7.3) und für die Messung einiger semantischer Parameter von Konstruktionen bilden. Dies ist die wesentliche Motivation für die in diesem Kapitel aufzuzeigenden konstruktikographischen Generalisierungen. Da lexikalische Frames und lexikalische Bedeutungen insbesondere für die im Folgenden aufgelisteten semantischen Parameter von Relevanz sind, finden Differenzierungen nach lexikalischen Frames und lexikalischen Bedeutungen zuvorderst im Rahmen der Verarbeitung dieser Parameter ihren Platz. Semantische Parameter, die auf lexikalischen Frames und lexikalischen Bedeutungen beruhen (vgl. auch Unterabschnitt 4.2.1), sind:

- konstruktionelle Polysemie (für den Fall, dass die Polysemie der Konstruktion, wie in Unterabschnitt 5.2.2 definiert, über eine Verteilung lexikalischer Frames und lexikalischer Bedeutung konstituiert wird und nicht bereits über unterschiedliche Konstruktions-Frames);

- Beschränkungen und Präferenzen (sofern sich diese auf lexikalische Frames und lexikalische Bedeutungen und damit nicht nur rein formale Aspekte beziehen); 
- Frame-Nähe (da das System der Frame-Nähen zum Konstruktions-Frame über dessen Frame-zu-Frame-Relationen zu anderen Frames definiert wird, die als lexikalische Frames infrage kommen können);

- Koerzionspotenzial (das wesentlich von lexikalischen Frames und lexikalischen Bedeutungen abhängt, worauf ich in Abschnitt 7.4 zurückkomme);

- Produktivität (die ebenfalls über lexikalische Frames, genauer: deren FrameNähe und Type-Frequenz, gemessen wird, vgl. Abschnitt 7.1.3);

- emergente Struktur (deren Verteilung sich über lexikalische Frames und die Konstitution von Konstrukt-Frames sowie lexikalische Bedeutungen ermitteln lässt, vgl. Abschnitt 7.6).

Ebenso wie lexikalische Frames und lexikalische Bedeutungen sind KonstruktFrames und Konstruktbedeutungen für die Messung einiger semantischer Parameter relevant und finden somit zumindest indirekt Eingang in einen Konstruktionseintrag. Da ich im Laufe dieses Kapitels noch auf die Messung dieser semantischen Parameter eingehe, sei an dieser Stelle kurz auf die Relevanz von KonstruktFrames und Konstruktbedeutungen dafür hingewiesen.

- Die Messung des Koerzionspotenzials (Abschnitt 7.4) beruht auf der Verteilung unterschiedlicher Varianten der Konstitution von Konstrukt-Frames, also dem Verhältnis zwischen lexikalischem Frame und Konstruktions-Frame bei der semantischen Motivierung von KtE und KEE. Eine Feindifferenzierung erfolgt dabei über variierende Konstruktbedeutungen.

- Die Verteilung einer emergenten Struktur und deren Messung (Abschnitt 7.6) beruht insbesondere auf Konstruktbedeutungen, die Ergebnis der unterschiedlichen Varianten der Evokation einer emergenten Struktur sind, wie ich sie in Unterabschnitt 5.7.2 differenziert habe.

Die in Unterabschnitt 7.1.1 zusammengestellte vorläufige Liste an Inhalten eines Konstruktionseintrages ist somit auf die Verarbeitung des konstruktionssemantischen Modells zumindest teilweise vorbereitet, da der Konstruktions-Frame darin bereits Eingang findet. Wie die anderen beiden Frame- und Bedeutungstypen innerhalb der semantischen Parameter von Konstruktionen in einem Konstruktionseintrag verarbeitet werden, ist Gegenstand des folgenden Unterabschnitts 7.1.3.

\subsubsection{Semantische Parameter in einem Konstruktionseintrag}

Die vorläufige Liste an Inhalten eines Konstruktionseintrags, die ich in Unterabschnitt 7.1.1 aus der konstruktikographischen Forschungsliteratur zusammengestellt habe, enthält noch nicht die semantischen Parameter, die einen zentralen 
Tab. 7.2: Verarbeitung der semantischen Parameter von Konstruktionen in einem Konstruktionseintrag

\begin{tabular}{ll}
\hline $\begin{array}{l}\text { Parameter ohne eigenen Datenpunkt im Kon- } \\
\text { struktionseintrag }\end{array}$ & $\begin{array}{l}\text { Parameter mit eigenem Datenpunkt im Kon- } \\
\text { struktionseintrag }\end{array}$ \\
\hline 1. Formale Abstraktheit & $\begin{array}{l}\text { 5. Koerzionspotenzial } \\
\text { 2. Konstruktionelle Polysemie }\end{array}$ \\
$\begin{array}{l}\text { 3. Beschroduktivität } \\
\text { 4. Frame-Nähe }\end{array}$ & 7. Emergente Struktur \\
\hline
\end{tabular}

Bestandteil des vorliegenden Entwurfs einer Konstruktionssemantik ausmachen und die ich in Kapitel 5 thematisiert habe. Wie müssen diese semantischen Parameter Eingang in einen Konstruktionseintrag finden?

Die Gliederungspunkte erster Ordnung in der in Unterabschnitt 7.1.1 zusammengestellten vorläufigen Liste der Inhalte eines Konstruktionseintrags können jeweils als eigene Datenpunkte gelten. Jeder der Punkte entspricht einem Informationsabschnitt in einem Konstruktionseintrag. Die Liste ist somit als Gliederung eines Konstruktionseintrags zu verstehen. Bei der Frage, wie die semantischen Parameter in diese Liste eingehen können, bestehen deshalb zwei Möglichkeiten. 1. Ein semantischer Parameter bekommt einen eigenen Datenpunkt in einem Konstruktionseintrag und ist somit Teil von dessen Gliederung.

2. Die Ergebnisse der Analyse eines semantischen Parameters fließen in die in einem Datenpunkt präsentierten Informationen ein (ggf. indem sie ihn binnendifferenzieren), der semantische Parameter selbst erhält keinen eigenen Datenpunkt im Konstruktionseintrag.

Die Frage nach der Verarbeitung der semantischen Parameter ist somit vorrangig eine Frage danach, welche Parameter einen eigenen Datenpunkt erhalten und welche lediglich in einen bereits bestehenden Datenpunkt einfließen. Die sieben in Kapitel 5 diskutierten semantischen Parameter lassen sich nach diesen beiden Kategorien differenzieren. Wie aus Tabelle 7.2 deutlich wird, möchte ich vorschlagen, dass die semantischen Parameter der formalen Abstraktheit, der konstruktionellen Polysemie, der Beschränkungen und Präferenzen sowie der FrameNähe keinen eigenen Datenpunkt in einem Konstruktionseintrag erhalten, während die semantischen Parameter des Koerzionspotenzials, der Produktivität sowie der emergenten Struktur einen solchen Datenpunkt erhalten. Ich möchte diese Aufteilung im Folgenden begründen, um dann in den Abschnitten 7.2 bis 7.6 der Reihe nach ihre Ergebnisse für die drei untersuchten Konstruktionen einzuordnen und deren Umsetzung in einem Konstruktionseintrag vorzubereiten. 
Der semantische Parameter der formalen Abstraktheit erhält keinen eigenen Datenpunkt, weil seine Ergebnisse bereits in einem bestehenden Parameter verarbeitet werden können. Die Liste aus Unterabschnitt 7.1.1 hält unter Gliederungspunkt 3 explizit eine schematische Angabe der formalen Struktur bereit, mit der die formale Abstraktheit der Konstruktion bereits angedeutet wird. Neben den beteiligten Phrasentypen und den lexikalisch fixierten Elementen, letzlich also der formalen Darstellung aller Strukturelemente der Konstruktion muss als Unterpunkt zu Gliederungspunkt 3 also noch die formale Abstraktheit der Konstruktion im Sinne des Verhältnisses aus KE, KEE und KorE, wie ich es in Abschnitt 5.1 definiert habe, ergänzt werden.

Der semantische Parameter der konstruktionellen Polysemie erhält ebenfalls keinen eigenen Datenpunkt in einem Konstruktionseintrag. Dies gilt für beide in Abschnitt 5.2 definierten Fälle von konstruktioneller Polysemie, also sowohl für den Fall, dass sie über unterschiedliche Konstruktions-Frames konstituiert wird (Unterabschnitt 5.2.1), als auch für den subtileren und für die drei untersuchten Konstruktionen einschlägigen Fall, dass sie sich über Varianzen in den lexikalischen Frames und lexikalischen Bedeutungen differenziert (Unterabschnitt 5.2.2). Konstituiert sich Polysemie über unterschiedliche Konstruktions-Frames, schlagen sich die Ergebnisse dieser Analyse ohnehin in unterschiedlichen Konstruktionseinträgen nieder, die möglicherweise die entsprechenden Frames bereits in den Konstruktionsnamen tragen können (dazu Unterabschnitt 7.2.1). Differenziert sich konstruktionelle Polysemie über lexikalische Frames und lexikalische Bedeutungen, muss diese Information selbstverständlich in den Konstruktionseintrag aufgenommen werden, allerdings zähle ich sie zu der Definition der Konstruktion (Gliederungspunkt 5 auf der Liste in Unterabschnitt 7.1.1), weshalb sie in den diesbezüglichen Datenpunkt Eingang finden müssen. Sie ist also als Unterpunkt dieses Informationsabschnitts zu verstehen. Sinnvoll ist, die konstruktionelle Polysemie an dieser Stelle bereits durch annotierte Beispielbelege zu illustrieren.

Die Beschreibung von Beschränkungen und Präferenzen, die den nächsten semantischen Parameter darstellen, ist an mindestens zwei Stellen der Liste in Unterabschnitt 7.1.1 bereits angelegt. Da sie die einzelnen Strukturelemente einer Konstruktion betreffen, gehören sie zu den Punkten 6, 7 und 8. Zu den KE und ihren Definitionen gehören Beschränkungen und Präferenzen in wesentlichem Maße, sie betreffen sowohl den Namen des KE (vgl. dazu ausführlicher Abschnitt 7.3) als auch seine Definition und formale Realisierung. Gleiches gilt für KEE und KorE.

Der letzte semantische Parameter, der keinen eigenen Datenpunkt enthält, betrifft die Frame-Nähen des Konstruktions-Frames. Auch die mit ihm verbundenen Informationen sind bereits an einigen Stellen des Konstruktionseintrags enthalten. Da sich das System der Frame-Nähen um den Konstruktions-Frame zentriert, 
der bereits in Punkt 4 der Liste in 7.1.1 angesprochen wird, kann es dem Datenpunkt, der für den Konstruktions-Frame vorgesehen ist, zugeordnet werden. Zudem gehören Angaben über Frame-Nähen zu Präferenzen und Beschränkungen, die, wie soeben erläutert, ebenfalls bereits im Konstruktionseintrag abgedeckt sind. Da Frame-Nähen entscheidend dazu beitragen, Konstrukte einer Konstruktion zu identifizieren und konstruktionelle Ambiguität aufzulösen (dazu Unterabschnitt 8.4.2), gehören Angaben über sie zu den Präferenzen eines KE, im Falle der drei untersuchten Konstruktionen jeweils dem KE EREIGNIS. Darüber hinaus fließen Informationen über Frame-Nähen in mindestens zwei weitere semantische Parameter ein, das Koerzionspotenzial und die Produktivität, die als eigene Datenpunkte in einen Konstruktionseintrag Eingang finden.

Die drei bisher noch nicht besprochenen semantischen Parameter finden sich in der Liste in Unterabschnitt 7.1.1 nicht wieder. Sie müssen deshalb als eigene Datenpunkte und damit eigene Informationsabschnitte in einen Konstruktionseintrag eingehen. Im Folgenden sei eine um diese drei semantischen Parameter (Koerzionspotenzial, Produktivität und emergente Struktur) sowie die eben diskutierte Verarbeitung der anderen vier Parameter erweiterte Liste zusammengestellt. Diese Liste ist Ausgangspunkt für die im Zusatzmaterial dargestellten Konstruktionseinträge zu den drei untersuchten Konstruktionen.

1. Konstruktionsname: Die Bezeichnung der Konstruktion, die bereits im Titel des Konstruktionseintrags enthalten ist.

2. Repräsentatives Beispielkonstrukt: Ein ausgewählter Beleg, der ein Konstrukt enthält, das zur schnellen Illustration der Konstruktion dient.

3. Schematische Angabe der formalen Struktur: Eine Darstellung der formalen Struktur der Konstruktion, die sich aus den folgenden drei Komponenten zusammensetzt.

- Beteiligte Phrasentypen: Die Phrasentypen, als die die KtE der einzelnen KE sowie KEE und KorE realisiert werden.

- Lexikalisch fixierte Elemente: Die KEE und KorE der Konstruktion, wobei sie nicht zwingend als feste LE angegeben werden müssen, sondern auch Kategorien umfassen können, etwa Reflexiva bei allen drei untersuchten Konstruktionen, Partikeln bei der reflexiven Partikelverbkonstruktion oder eine Bandbreite von Artikeln und Nomen bei der reflexiven WegKonstruktion.

- Formale Abstraktheit: Angabe des Verhältnisses zwischen KE (frei instanziierbaren Strukturelementen) und KEE sowie KorE (lexikalisch fixierten Strukturelementen).

4. Konstruktions-Frame: Der Frame, der als Konstruktions-Frame der Konstruktion gelten kann, im Falle der drei untersuchten Konstruktionen also Motion. 
- Frame-Nähen: Auflistung des Systems der Frame-Nähen des Konstruktions-Frames.

5. Definition der Konstruktion: Eine kurze allgemeine Charakterisierung der Konstruktion, ihrer formalen und semantischen Eigenschaften.

- Konstruktionelle Polysemie: Im Falle einer polysemen Konstruktion Angaben über die Art der Polysemie durch unterschiedliche KonstruktionsFrames oder Varianzen in lexikalischen Frames und lexikalischen Bedeutungen. Annotierte Beispielbelege zur Illustration der Polysemie.

6. KE und deren Definitionen: Eine Liste der KE der Konstruktion inklusive ihrer formalen und semantischen Eigenschaften, die die folgenden Komponenten umfassen.

- Name des KE: Die Bezeichnung des KE.

- Definition des KE: Eine kurze Definition des KE.

- Formale Realisierung des KE: Angaben zur formalen Realisierung des KE, bei Argumentstruktur-Konstruktionen wie den drei untersuchten insbesondere in Form von Phrasentypen.

- Beschränkungen und Präferenzen: Formale sowie semantische Beschränkungen und Präferenzen des KE, z.B. hinsichtlich der durch seine KtE evozierten lexikalischen Frames (etwa bei dem KE EREIGNIS der drei untersuchten Konstruktionen).

- Annotierte Beispielbelege: Zu jedem KE sollte mindestens ein allein auf dieses KE hin annotierter Beispielbeleg zur Illustration gehören.

7. KEE und deren Definition(en): Die Liste des bzw. der KEE und eine kurze Beschreibung seiner/ihrer formalen und semantischen Eigenschaften.

- Beschränkungen und Präferenzen: Formale sowie semantische Beschränkungen und Präferenzen von KEE, z.B. hinsichtlich möglicher Evokationen eines Konstruktions-Frames (vgl. dazu Unterabschnitt 8.3.1).

8. KorE und deren Definition(en): Die Liste des bzw. der KorE und eine kurze Beschreibung seiner/ihrer formalen und semantischen Eigenschaften.

- Beschränkungen und Präferenzen: Formale sowie semantische Beschränkungen und Präferenzen von KorE, z.B. hinsichtlich möglicher Evokationen eines Konstruktions-Frames (vgl. dazu Unterabschnitt 8.3.2).

9. Koerzionspotenzial: Darstellung der Zusammensetzung unterschiedlicher Koerzionsstufen und deren Illustration durch Beispielbelege. Angaben über das Koerzionspotenzial der Konstruktion im Vergleich zum Koerzionspotenzial anderer (verwandter) Konstruktionen.

10. Produktivität: Angaben über die Produktivität der Konstruktion im Vergleich zur Produktivität anderer (verwandter) Konstruktionen. Differenzierung relatierter und unrelatierter lexikalischer Frames sowie Angabe deren jeweiliger 
Type-Frequenz. Auflistung der belegten unrelatierten lexikalischen Frames analog zum System der Frame-Nähen.

11. Emergente Struktur: Beschreibung einer möglichen emergenten Struktur, die in den Konstrukten der Konstruktion evoziert werden kann, einschließlich entsprechender Voraussetzungen, die dafür gegeben sein müssen.

12. Relationen zu anderen Konstruktionen: Relationen zu anderen Konstruktionen im Konstruktikon. Diese können anstelle von strikten Vererbungsrelationen auch als Familienähnlichkeiten (vgl. Unterabschnitt 3.2.3) beschrieben werden.

- Formale Relationen: Formale Relationen bzw. Familienähnlichkeiten zu anderen Konstruktionen.

- Semantische Relationen: Semantische Relationen bzw. Familienähnlichkeiten zu anderen Konstruktionen.

Da ich Informationen über die Abstraktheit der drei untersuchten Konstruktionen sowie das System der Frame-Nähen des Konstruktions-Frames Motion bereits in den Abschnitten 5.1 und 5.4 diskutiert habe, konzentriere ich mich für den weiteren Verlauf dieses Kapitels auf diejenigen semantischen Parameter, deren Ergebnisse bisher noch nicht präsentiert wurden und die insbesondere als eigene Datenpunkte in einen Konstruktionseintrag eingehen müssen. Zuvor jedoch sollen einige allgemeine konstruktikographische Beschreibungen im Vordergrund stehen, die bisher ebenfalls noch nicht eingehend betrachtet wurden, in der oben zusammengestellten nun endgültigen Liste der Inhalte eines Konstruktionseintrags aber enthalten sind.

\subsection{Allgemeine konstruktikographische Beschreibungen}

Nachdem geklärt ist, welche Inhalte ein Konstruktionseintrag enthalten muss und wie das konstruktionssemantische Modell sowie die semantischen Parameter von Konstruktionen darin verarbeitet werden können, kann die eigentliche konstruktikographische Analyse, die ich für den restlichen Verlauf dieses Kapitels mit einigen weiteren methodischen Vorschlägen verknüpfen werde, beginnen. Für die drei untersuchten Konstruktionen kann die in Unterabschnitt 7.1.3 zusammengestellte finale Liste der Inhalte eines Konstruktionseintrages nun gewissermaßen ,von oben nach unten' abgearbeitet werden. Dabei sollen alle Informationen, die nicht trivialer Natur sind (wie etwa die schematische Angabe der formalen Struktur) in den Blick genommen werden.

In diesem Abschnitt stehen zunächst zwei allgemeine konstruktikographische Informationen im Vordergrund. In Unterabschnitt 7.2.1 betrachte ich das 
bisher noch undiskutiert gebliebene Zustandekommen der Konstruktionsnamen der drei Konstruktionen, mit denen jeder Konstruktionseintrag beginnt. In Unterabschnitt 7.2.2 schließlich soll noch einmal die Differenzierung konstruktioneller Polysemie für die drei Konstruktionen angesprochen und ihre Form für die Verarbeitung in Konstruktionseinträgen festgelegt werden.

\subsubsection{Konstruktionsnamen}

Jeder Konstruktionseintrag beginnt mit dem Konstruktionsnamen, also der Bezeichnung der Konstruktion, die in dem betreffenden Eintrag beschrieben wird. Die Wahl des Konstruktionsnamens ist deshalb ausschlaggebend für die präzise Dokumentation der Konstruktion in einem Konstruktikon, da der Konstruktionsname möglichst schon vor einem Blick auf die Inhalte des Konstruktionseintrags erste einschlägige Informationen vermitteln soll. Die verschiedenen Varianten, eine Konstruktion zu benennen, sind bisher kaum expliziter Gegenstand der konstruktikographischen Forschung, weshalb ich im Folgenden anhand der drei untersuchten Konstruktionen einige Prinzipien der Wahl eines Konstruktionsnamens diskutieren möchte. Da ich die Wahl der Konstruktionsnamen für die drei Konstruktionen im bisherigen Verlauf der Arbeit noch nicht thematisiert habe, bietet sich an dieser Stelle eine entsprechende Reflexion an.

Gemäß der konstruktionsgrammatischen Auffassung einer Konstruktion als ,Form-Bedeutungs-Paar` (Unterabschnitt 2.2.1) können sich in einem Konstruktionsnamen sowohl Aspekte der Formseite einer Konstruktion als auch Aspekte ihrer semantischen Eigenschaften wiederfinden: „,[D]ie Namen der Konstruktionen sind sprechend und sollen die Bedeutung oder Funktion einer Konstruktion widerspiegeln, insofern dies möglich und sinnvoll ist.“ (Ziem 2020a: 25-26). Die drei untersuchten Konstruktionen kombinieren in ihren Konstruktionsnamen teils beide miteinander und zeigen, dass sich diese nicht gegenseitig ausschließen, sondern gleichzeitig Eingang in einen Konstruktionsnamen finden können.

Der Konstruktionsname reflexive Bewegungskonstruktion, den schon Oya (1999: 358) verwendet, verweist wie die bei Perek \& Hilpert (2014: 270) und Boas \& Ziem (2018b: 211) zu findende Bezeichnung reflexive-motion construction zugleich auf formale und semantische Aspekte. Das Attribut reflexiv, das für alle drei untersuchten Konstruktionen, also auch für die reflexive Partikelverbkonstruktion und die reflexive Weg-Konstruktion gilt, stellt eine formale Beschränkung jeweils eines Strukturelements der drei Konstruktionen heraus (dazu Unterabschnitt 5.3.2). Da alle drei Konstruktionen formal als Reflexivkonstruktionen einzustufen sind (vgl. Unterabschnitt 3.1.5), verweist das Attribut reflexiv darauf, dass ein Reflexivum als KEE obligatorisch ist. 
Die Wahl des Ausdrucks, der im Konstruktionsnamen auf das Attribut reflexiv folgt, also Bewegungskonstruktion, Partikelverbkonstruktion und Weg-Konstruktion, ist für die drei untersuchten Konstruktionen weniger trivial. Die Bezeichnung Bewegungskonstruktion verweist offensichtlich auf eine semantische Eigenschaft der Konstruktion, nämlich auf Motion als ihren Konstruktions-Frame. Freilich kommt diese Eigenschaft in der englischen Bezeichnung motion construction noch deutlicher zum Ausdruck. Als Alternative böte sich an, den FrameNamen direkt in der Konstruktion zu kennzeichnen, die Konstruktion also als reflexive Motion-Konstruktion zu bezeichnen. Da diese Konventionen in der nichtkonstruktikographischen Forschung, die auch keine Bezüge zu FrameNet kennt, allerdings intransparent wäre, stellt der Konstruktionsname reflexive Bewegungskonstruktion die sinnvollere Alternative dar.

Indes ist der Einbezug eines Frame-Namens in den Konstruktionsnamen bereits eine bekannte Methode, um insbesondere polyseme Konstruktionen, deren Polysemie über unterschiedliche Konstruktions-Frame konstituiert wird (Unterabschnitt 5.2.1) zu differenzieren. Genau diesen Weg gehen für die Familie der englischen V-that-Konstruktionen Perek \& Patten (2019: 381): „Except for the Relation ' $V$ that' construction, these constructions evoke the frame mentioned in their name“. Beruht die Polysemie einer Konstruktion auf unterschiedlichen Konstruktions-Frames, bietet es sich also an, diese in die Konstruktionsnamen aufzunehmen, da, wie in Unterabschnitt 5.2.1 argumentiert, dafür mehrere Konstruktionseinträge mit unterschiedlichen Konstruktionsnamen angelegt werden müssen. Am Beispiel der Subjekt-Auxiliar-Inversion ließen sich für die in Tabelle 5.1 (Unterabschnitt 5.2.1) aufgelisteten Lesarten nach Goldberg (2006: 177) Konstruktionseinträge mit den folgenden Konstruktionsnamen anlegen:

- Questioning-Subjekt-Auxiliar-Inversion;

- Conditional_scenario-Subjekt-Auxiliar-Inversion;

- Desiring-Subjekt-Auxiliar-Inversion;

- Experiencer_obj-Subjekt-Auxiliar-Inversion.

Während die reflexive Bewegungskonstruktion in ihrem Namen neben der Klassifizierung als Reflexivkonstruktion nun also auf semantische Eigenschaften hinweist, stellt sich dies bei der reflexiven Partikelverbkonstruktion und der reflexiven Weg-Konstruktion anders dar. Sie beide verweisen nicht nur mit dem Attribut reflexiv auf formale Beschränkungen der jeweiligen Konstruktionen, sondern ebenso durch den restlichen Konstruktionsnamen. Dies ist aufgrund der Verwandtschaft zur reflexiven Bewegungskonstruktion begründungsbedürftig.

Die reflexive Partikelverbkonstruktion verweist mit dem Ausdruck Partikelverbkonstruktion ähnlich wie durch das Attribut reflexiv auf die formale Eigenschaft eines ihrer Strukturelemente, diesmal des KE EREIGNIS. Wie in den Un- 
terabschnitten 3.2.2 und 5.3.2 ausgeführt, liegt auf diesem KE die Beschränkung, dass es durch das Basisverb eines Partikelverbs instanziiert werden muss, was zugleich zu der Annahme eines zweiten KEE, nämlich RICHTUNG, führt, das durch die korrespondierende Verbpartikel instanziiert wird. Obwohl die reflexive Bewegungskonstruktion ebenfalls Partikelverben als KtE ihres KE EREIGNIS erlaubt - wodurch eine Überschneidung mit der reflexiven Partikelverbkonstruktion zustande kommt (vgl. Unterabschnitt 3.2.3) -, wirkt die Beschränkung auf Kombinationen von KtE des KE EREIGNIS mit der Instanziierung des KEE RICHTUNG unmittelbar auf Eigenschaften anderer KE zurück. So ist das KE 〈WEG〉 der reflexiven Partikelverbkonstruktion als Nicht-Kern-KE zu klassifizieren, weil es allein dann null-instanziiert werden kann, wenn die Kombination eines KtE des KE EREIGNIs mit der Instanziierung des KEE RICHTUng ein Partikelverb ergibt (vgl. Unterabschnitt 6.4.2). Zudem ist die formale Varianz der KtE des KE 〈WEG), die nicht nur die Form einer PP, sondern auch einer NP und eines Nebensatzes annehmen können, allein durch die ein Partikelverb ergebende Kombination eines KtE des KE EREIGNIS mit der Instanziierung des KEE RICHTUnG möglich. Die reflexive Bewegungskonstruktion verfügt über diese Eigenschaften des KE WEG nicht, da ihr KE EREIGNIS überwiegend durch simplizische Verben instanziiert wird, die mit einer Varianz der KtE wie derjenigen des KE 〈WEG〉 der reflexiven Partikelverbkonstruktion nicht kompatibel sind (vgl. weiterhin Unterabschnitt 3.2.3).

Aus ebendiesem Grund erscheint es sinnvoll, die Beschränkung des KE EREIGNIS auf Basisverben von Partikelverben in den Konstruktionsnamen zu übernehmen, wenngleich dahinter semantische Eigenschaften der Konstruktion zurücktreten. Darüber hinaus ist die Betonung des Attributs reflexiv in Verbindung mit der formalen Spezifizierung Partikelverbkonstruktion für die argumentstrukturelle Einordnung dieser Konstruktion relevant. Dass Konstruktionen wie diese bisweilen ignoriert werden, zeigt der Überblick von McIntyre (2007), der eine explizite Klassifikation der Argumentstrukturen von Partikelverben (für alle germanischen Sprachen) zum Ziel hat, Reflexivkonstruktionen dabei aber unberücksichtigt lässt. ${ }^{8}$

Ähnliche Gründe wie für die reflexive Partikelverbkonstruktion gelten schließlich für die reflexive Weg-Konstruktion. Ihr Name, den bereits Verhagen (2003a:

8 Die Begründung lautet: „The survey ignores interactions between particles and external arguments because there apparently are none.“ (McIntyre 2007: 365, Anm. 8). Dies allerdings kann zumindest für das Deutsche nicht geltend gemacht werden, zumal McIntyre - konträr zu seinem Anspruch - ausschließlich mit dem Englischen argumentiert. 
338, 2003c: 228) verwendet, ${ }^{9}$ ist unverkennbar mit demjenigen der way-Konstruktion $\mathrm{zu}$ parallelisieren, was aus sprachvergleichender Sicht ebenfalls formale Gründe hat. Die reflexive Weg-Konstruktion nämlich entspricht hinsichtlich ihrer Strukturelemente nahezu direkt der way-Konstruktion (vgl. Unterabschnitt 3.3.2), eine mögliche Motivation dafür, warum sie bisweilen, etwa von McColm (2019: 38-42), als vorrangiges Äquivalent zur way-Konstruktion behandelt wird. Im Zusammenhang mit dieser sprachvergleichenden Dimension verweist der Konstruktionsname der reflexiven Weg-Konstruktion auf ihr formales Charakteristikum eines KorE, das zu dem für die anderen beiden Konstruktionen obligatorischen KEE hinzutritt und eine NP mit der LE Weg oder semantisch verwandter LE als Kopf umfasst, die analog für die way-Konstruktion und die LE way gilt. Von der reflexiven Bewegungskonstruktion unterscheidet sich die reflexive Weg-Konstruktion hinsichtlich ihrer Strukturelemente einzig durch dieses zusätzliche KorE (neben der Tatsache, dass das KEE als Reflexivum im Dativ realisiert wird). Da dieses formale Charakteristikum für die reflexive Weg-Konstruktion entscheidend ist, erscheint es wie bei der reflexiven Partikelverbkonstruktion gerechtfertigt, es zu Ungunsten semantischer Eigenschaften im Konstruktionsnamen zu verankern.

Die Verwandtschaft zwischen den drei Konstruktionen (Unterabschnitte 3.1.5 und 3.2.3) spiegelt sich also, dies ist ein weiterer nicht unwesentlicher Aspekt, nicht in vollem Umfang in deren Konstruktionsnamen wider - lediglich die Klassifizierung als Reflexivkonstruktionen ist ihnen in dieser Hinsicht gemein. Ihre semantische Verwandtschaft kann erst durch Bezüge zwischen den Konstruktionen innerhalb eines Konstruktikons hergestellt werden, etwa durch die Zuordnung von Motion als gemeinsamem Konstruktions-Frame. Die eben erwähnte Notwendigkeit zur Kennzeichnung charakteristischer Eigenschaften bereits im Konstruktionsnamen muss also freilich durch den eigentlichen Inhalt des Konstruktionseintrags komplettiert werden, der den Kerngegenstand der konstruktikographischen Beschreibung ausmacht.

\subsubsection{Differenzierung polysemer Konstruktionen}

Konstruktionelle Polysemie als Gegenstand eines semantischen Parameters von Konstruktionen (Abschnitt 5.2) zählt zu den allgemeinen konstruktikographischen Beschreibungen, weil sie bereits vor der Zusammenstellung des Inhalts eines Konstruktionseintrags über die Struktur der Konstruktionseinträge in einem

\footnotetext{
9 Die Bezeichnung „Weg-Konstruktion“ findet sich auch bei Smirnova (2018: 24) sowie im deutschen Abstract des Aufsatzes von Mortelmans \& Smirnova (2020: 47). Analog nutzen sie die englische Bezeichnung „Weg-construction“ (z.B. Mortelmans \& Smirnova 2020: 59).
} 
Konstruktikon entscheidet. Wie ich bereits gezeigt habe, lassen sich konstruktionssemantisch betrachtet (mindestens) zwei Arten von Polysemie unterscheiden:

a) konstruktionelle Polysemie durch unterschiedliche Konstruktions-Frames (Unterabschnitt 5.2.1);

b) konstruktionelle Polysemie durch variierende lexikalische Frames und lexikalische Bedeutungen (Unterabschnitt 5.2.2).

Wie ich ebenfalls im Zuge dieser Diskussion argumentiert habe, erscheint es hinsichtlich konstruktikographischer Generalisierungen gerechtfertigt, für Polysemie im Sinne von a unterschiedliche Konstruktionseinträge anzunehmen, während Polysemie im Sinne von b in einem einzigen Konstruktionseintrag zusammengefasst werden kann. In den Unterabschnitten 5.2.3 und 5.2.4 habe ich am Beispiel der reflexiven Bewegungskonstruktion argumentiert, dass die drei untersuchten Konstruktionen unter Polysemie im Sinne von b fallen. Sie verfügen über insgesamt vier Lesarten: eine neutrale Lesart sowie eine ,manner'-, eine ,means'- und eine ,incidental'-Lesart. Diese Lesarten müssen nicht in unterschiedlichen Konstruktionseinträgen ausdifferenziert werden, sondern können für jede Konstruktion innerhalb ihres Konstruktionseintrags zusammenfassend erläutert werden. Diese Beschreibung gehört, wie in Unterabschnitt 7.1.3 vorgeschlagen, zur Definition der jeweiligen Konstruktion und muss damit nicht als eigener Datenpunkt innerhalb eines Konstruktionseintrags angesetzt werden. Zur Illustration der Lesarten können jeweils, wie für die Illustration der Konstruktion als Ganzes, repräsentative Beispielkonstrukte dienen.

Eine für die konstruktikographische Beschreibung interessante Beobachtung, die ich um Zuge der Diskussion um die vier Lesarten in den Unterabschnitten 5.2.3 und 5.2.4 noch nicht reflektiert habe, ist die quantitative Verteilung der einzelnen Lesarten über die Konstrukte der drei Konstruktionen. Da diese ebenso wie etwa das Koerzionspotenzial (Abschnitt 7.4), die Produktivität (Abschnitt 7.5) und die emergente Struktur (Abschnitt 7.6) zu den Daten gehört, die eine konstruktikographische Generalisierung darstellen, möchte ich sie in diesem Unterabschnitt für die drei untersuchten Konstruktionen aufzeigen.

In den Tabellen 7.3 bis 7.5 sind die Verteilungen der Konstrukte der drei untersuchten Konstruktionen auf die vier Lesarten dargestellt. Es fällt auf, dass Konstrukte mit einer neutralen Lesart bei allen drei Konstruktionen die Mehrheit bilden. Beispiele für solche Konstrukte für die drei untersuchten Konstruktionen zeigen die drei Belege (1)-(3), jeweils mit Motion als lexikalischem Frame. 
Tab. 7.3: Verteilung der Lesarten der reflexiven Bewegungskonstruktion

\begin{tabular}{lrr}
\hline Lesart & Konstrukte & Anteil \\
\hline neutral & 557 & $55,09 \%$ \\
,manner & 336 & $33,23 \%$ \\
,means & 68 & $6,73 \%$ \\
,incidental & 50 & $4,95 \%$ \\
\hline Gesamt & 1.011 & $100,00 \%$ \\
\hline
\end{tabular}

Tab. 7.4: Verteilung der Lesarten der reflexiven Partikelverbkonstruktion

\begin{tabular}{lrr}
\hline Lesart & Konstrukte & Anteil \\
\hline neutral & 590 & $69,41 \%$ \\
,manner & 148 & $17,41 \%$ \\
,means' & 64 & $7,53 \%$ \\
,incidental & 48 & $5,65 \%$ \\
\hline Gesamt & 850 & $100,00 \%$ \\
\hline
\end{tabular}

(1) Die Indianerin [motion bewegte] sich in ihren flachen Schuhen beinahe geräuschlos durch das Treppenhaus, während die Badelatschen immer lauter gegen Esthers Hacken schlappten. (Düffel, John von: Houwelandt, Köln: DuMont Literatur und Kunst Verlag 2004, S. 128)

(2) Die Schlange [motion bewegte] sich ein paar Schritte vorwärts auf die Cafetür zu. (Boie, Kirsten: Skogland, Ort: Hamburg 2005, S. 28)

(3) Hier also hat sich die glühende Lava einst ihren Weg ins Freie [Motion gebahnt]. (Die Zeit, 09.03.2000, Nr. 11)

Ein Vergleich zwischen der reflexiven Bewegungskonstruktion und der reflexiven Partikelverbkonstruktion zeigt zudem, dass jeweils die ,manner'-Lesart quantitativ an zweiter Stelle steht, auf die dann die ,means'-Lesart an dritter Stelle folgt. Beleg (4) soll noch einmal die ,manner-Lesart für die reflexive Bewegungskonstruktion illustrieren, während Beleg (5) dies für die reflexive Partikelverbkonstruktion tut. Zum Vergleich ist der lexikalische Frame auch hier, wie in den Belegen (1) und (2), Motion. 
Tab. 7.5: Verteilung der Lesarten der reflexiven Weg-Konstruktion

\begin{tabular}{lrr}
\hline Lesart & Konstrukte & Anteil \\
\hline neutral & 26 & $96,30 \%$ \\
,manner‘ & 0 & \\
,means‘ & 1 & $3,70 \%$ \\
,incidental‘ & 0 & \\
\hline Gesamt & 27 & $100,00 \%$ \\
\hline
\end{tabular}

(4) Er [Motion schlängelt] sich vom Hermon-Gebirge zum See Genezareth und zum Toten Meer, durch ein Gebiet, das unter ständiger Trockenheit leidet. (o. A. [kago]: Jordan. In: Aktuelles Lexikon 1974-2000, München: DIZ 2000 [2000])

(5) Die Karkasse war an ihrer Unterseite aufgerissen, die Eingeweide waren hervorgequollen, [Motion schlängelten] sich auf und dümpelten im Wasser zwischen den Algen und dem Kelp. (Schrott, Raoul: Tristan da Cunha oder die Hälfte der Erde; Hanser Verlag 2003, S. 72)

Die beiden Belege (6) und (7) sind Beispiele für die ,means'-Lesart der reflexiven Bewegungskonstruktion und der reflexiven Partikelverbkonstruktion.

(6) [work Arbeitete] sich durch ein 14 Pfund schweres Handbuch, das er vom FBI aus Washington erhalten hatte (für die 70 Mark Luftfracht musste er seine Wirtin anpumpen), und schrieb ganz wie sein Vorbild Karl May über ein Land, das er nie mit eigenen Augen gesehen hatte. (Die Zeit, 06.04.2000, Nr. 15)

(7) Schließlich [shopping kaufte] sich AOL vergangenen Sommer bei der SatellitenTV-Firma Hughes Electronics ein. (Die Zeit, 13.01.2000, Nr. 3)

Für die reflexive Weg-Konstruktion ist neben der neutralen Lesart nur die ,means'Lesart mit einem einzigen Konstrukt belegt, das ich in (8) wiedergebe. Die anderen beiden Lesarten sind für sie nicht belegt.

(8) Mein Schälmesser mit der dünnen Klinge [cutting säbelt] sich einen Weg durch die buschigen Petersilienköpfe, während ich überlege, ob es tatsächlich Köpfe oder doch Blätter oder gar Büschel heißt. (Riedel, Susanne: Eine Frau aus Amerika, Berlin: Berlin Verlag 2003, S. 106) 
Die ,incidental'-Lesart liegt sowohl bei der reflexiven Bewegungskonstruktion als auch bei der reflexiven Partikelverbkonstruktion auf dem letzten Rang. Die Belege (9) und (10) sollen diese Lesart für diese beiden Konstruktionen illustrieren.

(9) Niemand hier kann was Konkretes, alle wurschteln bloß rum, saugen Comedy aus der Tragödie, albern in Wortspielen, [Prevarication juxen] sich durch Schillerbrocken und Kantinenwitze, mal mühsam, mal spaßig, immer bemüht - und manchmal führt das Bemühen zu Erfolgen, und man sieht ein, dass alle politischen Ränke nur von der Gier , auf irgendwas“ angetrieben und nur durch Gehirnleere der Menschen ermöglicht werden. (Die Zeit, 27.04.2000, Nr. 18)

(10) Die zweite Schöpfung, die in der Enterprise nicht bloß aus Kontroll- und Waffensystemen, sondern auch aus Computermenschen mit Identitätsproblemen besteht und den Angriffen der intergalaktischen Maschinenmenschen namens „Borg“ im Dienste der „authentischen“ Menschen widerstehen muss, [Progression entwickelt] sich konstant, aber höchst bedrohlich in die Zukunft hinein. (Die Zeit, 10.02.2000, Nr. 7)

Die Verteilung der Konstrukte, das geht aus den Tabellen 7.3 bis 7.5 hervor, folgt, zumindest für die reflexive Bewegungskonstruktion und die reflexive Partikelverbkonstruktion, quantitativ absteigend dieser Reihenfolge der Lesarten: neutral, ,manner', ,means' und ,incidental'. Für die reflexive Weg-Konstruktion ist aufgrund der wenigen Belege ein solches Muster nicht herauszustellen.

Interessant an diesen Beobachtungen ist, dass die Anzahl der Konstrukte mit höher werdender Spezifität der Lesarten teils deutlich abnimmt. Dies hängt nicht unwesentlich mit der semantischen Motivierung der Konstrukte, also der Konstitution des Konstrukt-Frames, zusammen. Wie in Unterabschnitt 5.2.3 festgehalten, lässt sich etwa die ,incidental'-Lesart darüber definieren, dass der Anteil des lexikalischen Frames in Konstrukten mit dieser Lesart äußerst gering ist und umgekehrt dem Konstruktions-Frame ein besonders hoher Anteil zukommt. An der vollständigen Annotation der oben in (9) und (10) zitierten Konstrukte lässt sich dies in (11) und (12) nachvollziehen. Nur die KtE der KE BEWEGENDES werden hier durch die lexikalischen Frames motiviert, während alle anderen Strukturelemente durch FE des Konstruktions-Frames einfach motiviert werden. Es handelt sich um Variante c einer Frame-Anpassung des lexikalischen Frames durch den Konstruktions-Frame (Unterabschnitt 4.4.1).

(11) Niemand hier kann was Konkretes, [SPEAKER alle] wurschteln bloß rum, saugen Comedy aus der Tragödie, albern in Wortspielen, [Prevarication juxen] $\left[\right.$ sich $^{\text {THEME}}$ ] [durch Schillerbrocken und Kantinenwitze ${ }^{\text {PATH}}$ ], mal mühsam, 
mal spaßig, immer bemüht - und manchmal führt das Bemühen zu Erfolgen, und man sieht ein, dass alle politischen Ränke nur von der Gier , auf irgendwas“ angetrieben und nur durch Gehirnleere der Menschen ermöglicht werden. (Die Zeit, 27.04.2000, Nr. 18)

(12) [Eмтіту Die zweite Schöpfung, die in der Enterprise nicht bloß aus Kontrollund Waffensystemen, sondern auch aus Computermenschen mit Identitätsproblemen besteht und den Angriffen der intergalaktischen Maschinenmenschen namens „Borg“ im Dienste der „authentischen“ Menschen widerstehen muss,] [Progression entwickelt] [sich THEME] konstant, aber höchst bedrohlich [in die Zukunft GoAL] [hinein Direction]. (Die Zeit, 10.02.2000, Nr. 7)

Im Gegensatz zu diesem Fall werden Konstrukte mit neutraler Lesart in der Regel allein durch den (relatierten) lexikalischen Frame motiviert (dazu Unterabschnitt 6.2.1), enthalten also häufig gar keinen Anteil des Konstruktions-Frames. Dies ist insbesondere dann der Fall, wenn der lexikalische Frame mit dem KonstruktionsFrame identisch ist, wie in den oben als (1)-(3) zitierten Belegen. Eine vollständige Annotation der dortigen Konstrukte soll dies in (13)-(15) verdeutlichen.

(13) [Theme Die Indianerin] [Motion bewegte] sich in ihren flachen Schuhen beinahe geräuschlos [РАтн durch das Treppenhaus], während die Badelatschen immer lauter gegen Esthers Hacken schlappten. (Düffel, John von: Houwelandt, Köln: DuMont Literatur und Kunst Verlag 2004, S. 128)

(14) [Tнеме Die Schlange] [Motion bewegte] sich ein paar Schritte vorwärts [GoAL auf die Cafetür] [Direction zu]. (Boie, Kirsten: Skogland, Ort: Hamburg 2005, S. 28)

(15) Hier also hat sich [Theme die glühende Lava] einst ihren Weg [GoAL ins Freie] [Motion gebahnt]. (Die Zeit, 09.03.2000, Nr. 11)

Die Schlussfolgerung, dass die Token-Frequenz mit steigendem Anteil des Konstruktions-Frames an der Konstitution des Konstrukt-Frames sinkt, ist allein aufgrund dieser Analysen lediglich als grobe Tendenz zu verstehen. Ob sie sich darüber hinaus empirisch rechtfertigen lässt, kann nur durch eine genauere Analyse der Anteile des Konstruktions-Frames an der Konstitution von Konstrukt-Frames entschieden werden, mit anderen Worten: durch die Untersuchung von Koerzionseffekten. Ich komme darauf in Abschnitt 7.4 im Zuge der Messung des Koerzionspotenzials zurück. 


\subsection{Benennung und Definition von KE und KEE}

Nach der allgemeinen konstruktikographischen Beschreibung einer Konstruktion, zu der die ersten fünf Punkte der in Unterabschnitt 7.1.3 zusammengestellten Liste gehören, die also den Konstruktionsnamen, ein repräsentatives Beispielkonstrukt, die schematische Angabe der formalen Struktur, die Angabe des Konstruktions-Frames und die Definition der Konstruktion samt Informationen über ihre Polysemie umfasst, ist die Beschreibung der Strukturelemente der Konstruktion ein zweiter wesentlicher Bestandteil eines Konstruktionseintrags. ${ }^{10}$ Dies gilt für alle Konstruktionen, deren Komplexität über mindestens zwei Strukturelemente bestimmt wird (vgl. Unterabschnitt 5.1.1) und die sich dadurch als syntaktische Konstruktionen kennzeichnen lassen (vgl. dazu Langacker 1987: 82; Diessel 2019: 11). Zu dieser Beschreibung der Strukturelemente zählen einerseits deren Benennung, also die Wahl eines sprechenden Namens etwa für ein KE (z.B. BEWEGENDES, EREIGNIS, WeG) und andererseits die Definition des Strukturelements, $\mathrm{zu}$ welcher Angaben über seine formale Realisierung ebenso gehören wie solche über Beschränkungen und Präferenzen. So argumentiert aus valenztheoretischer Perspektive etwa Herbst (2018b: 10-11), dass die Beschreibung der Komplemente einer Argumentstruktur-Konstruktion stets eine formale und eine semantische Charakterisierung umfassen muss. Damit sind explizit deren Strukturelemente angesprochen, mit denen aus konstruktikographischer Perspektive KE, KEE und KorE gemeint sind. Ihre Benennung und Definition ist von Relevanz, da insbesondere die KE einer Konstruktion „daughter signs of the construction“ (Laviola et al. 2017: 194), also eigenständige Zeichen sind, die sowohl über formale als auch semantische Eigenschaften verfügen. ${ }^{11}$

Über die Methoden, wie die „mnemonic labels“ (Fillmore, Lee-Goldman \& Rhomieux 2012: 330), mit denen in erster Linie KE, aber bisweilen auch KEE und KorE bezeichnet werden, zustande kommen, wird in der konstruktikographischen Forschung bislang kaum reflektiert. In Unterabschnitt 2.3.2 habe ich darauf hingewiesen, dass es bisweilen vorkommt, dass KE aufgrund ihrer schon terminologischen Parallele mit FE nicht selten nach Letzteren benannt werden. Boas, Lyngfelt \& Torrent (2019: 26) halten etwa für das FrameNet-Konstruktikon und dessen Umgang mit der way-Konstruktion fest, dass „the CEs of some constructions are

10 Gleiches gilt für die Beschreibung der FE eines Frames, vgl. dazu aus lexikalischer Perspektive Busse, Felden \& Wulf (2018: 340-345).

11 Diese Ansicht ist unverkennbar auf Langacker (1987: 91) zurückzuführen (vgl. das Zitat im Eingang zu Kapitel 6), dessen Annahme einer Auflösbarkeit der globalen symbolischen Relation in einer Konstruktion in kleinere symbolische Relationen ihrer einzelnen Strukturelemente ich im Eingang zu Kapitel 6 erwähnt habe. 
coincidental with the FEs of the frame they evoke, such as in the way_neutral cxn“. Dass dies allerdings eine allzu kurze Schlussfolgerung ist und KE (bzw. deren KtE) nicht invariant mit FE des Konstruktions-Frame gleichgesetzt werden können, haben die Analysen in Kapitel 6 gezeigt. Vor dem Hintergrund der variierenden semantischen Motivierung der Strukturelemente der Konstrukte einer Konstruktion (KtE und KEE, potenziell aber auch KorE) durch die FE von lexikalischem Frame und Konstruktions-Frame ist klar, dass die Parallelen zwischen KE und FE nicht nur in der konkreten Analyse von Konstrukten, sondern auch für die Generalisierung dieser Analysen hin zu einem Konstruktionseintrag und damit für die Benennung und Definition der Struktuelemente einer Konstruktion differenziert betrachtet werden müssen.

Die Annahme, dass KE strikt analog zu FE benannt und semantisch definiert werden können, ist daher zu differenzieren. Wird, wie in den Einträgen für die wayKonstruktion im FrameNet-Konstruktikon, dafür schlicht und ausschließlich auf die FE des Konstruktions-Frames zurückgegriffen, also auf Motion, verschleiert dies die unterschiedlichen Verhältnisse der semantischen Motivierung von Konstrukten und damit der Konstitution von Konstrukt-Frames, die bisweilen keinen Anteil eines separaten Konstruktions-Frames enthalten müssen, wenn der lexikalische Frame relatiert ist (vgl. Unterabschnitt 6.2.1). Darüber hinaus verschleiert es den öfter auftretenden Fall, dass FE des Konstruktions-Frames regelmäßig mit FE eines lexikalischen Frames fusionieren, wodurch eine doppelte Motivierung eines KtE oder KEE (Unterabschnitte 6.2.3 und 6.3.3) entsteht. Bereits diese Gründe sprechen dafür, dass die variierenden Verhältnisse der semantischen Motivierung von KtE und KEE durch lexikalische Frames und den Konstruktions-Frame in die Definition von KE und KEE mit einfließen müssen. Die Betonung liegt darauf, dass darin dass KEE mit eingeschlossen werden muss, wofür die drei untersuchten Konstruktionen ein anschauliches Beispiel sind, da sich die Varianten in der semantischen Motivierung nicht nur auf die KtE von KE beschränken, sondern das jeweilige KEE einer Konstruktion ebenso einschließen. ${ }^{12}$ Seine semantische Motivierung variiert - zumindest für die reflexive Bewegungskonstruktion und die reflexive Partikelverbkonstruktion - fast ebenso stark wie diejenige der KtE eines KE.

Bei alledem darf nicht unberücksichtigt bleiben, dass die Benennung und Definition von KE und KEE auf der Grundlage von FE nicht die einzige Möglichkeit

12 Das KorE der reflexiven Weg-Konstruktion schließe ich, wie bereits in Unterabschnitt 6.1.1 begründet, aus, weil es, noch stärker als dessen KEE, als frame-evozierend angesehen werden kann und hierfür nur der Konstruktions-Frame infrage kommt (vgl. Unterabschnitte 8.3.1 und 8.3.2). Dieser Ausschluss des KorE gilt allerdings nicht grundsätzlich, sondern ist ein Charakteristikum der reflexiven Weg-Konstruktion. 
ist. Sie ist vielmehr auf bestimmte Strukturelemente beschränkt. So lassen sich für die drei untersuchten Konstruktionen etwa lediglich KE wie BEWEGENDES und WEG bzW. 〈WEG〉 über FE bestimmen, nicht aber EREIGNIS, da es für alle drei Konstruktionen durch eine den lexikalischen Frame evozierende LE instanziiert wird, die selbst nicht durch FE motiviert werden kann. Darüber hinaus hängen die Möglichkeiten zur Benennung und Definition eines Strukturelements, wenn man sie mit der Hilfe von FE betreibt, stark von jenem fokussierten Strukturelement und seiner Funktion innerhalb der Konstruktion ab. Auf all diese Aspekte möchte ich in diesem Abschnitt eingehen, einige methodische Herangehensweisen diskutieren und für die drei untersuchten Konstruktionen anwenden.

Ich beschränke mich dabei auf die Benennung und Definition der KE EREIGNIS, BEWEGENDES und WEG bzw. 〈WEG〉. Für die als Reflexivum instanziierten KEE aller drei untersuchten Konstruktionen erübrigt sich aus offensichtlichen Gründen eine Diskussion um ihre Benennung, durch ihre Natur als KEE lassen sich außerdem (formale) Beschränkungen einfacher formulieren (vgl. Unterabschnitt 5.3.2). Zudem ist ihre semantische Motivierung ohnehin eingeschränkter als etwa diejenige der KtE des KE WEG bzw. 〈WEG〉, da sie für lexikalische Frames nur dann untersuchbar ist, wenn es sich um kausative Frames handelt (vgl. Unterabschnitt 6.3.1). Die Benennung des KE RICHTUNG der reflexiven Partikelverbkonstruktion habe ich bereits in Unterabschnitt 6.4.2 begründet.

In Unterabschnitt 7.3.1 gehe ich auf den Fall ein, in dem ein KE nicht über FE benannt und definiert werden kann und für den das KE EREIGNIS der drei untersuchten Konstruktionen ein Beispiel ist. Ausgehend davon soll in Unterabschnitt 7.3.2 mit besonderem Fokus auf das KE BEwEGENDES die Möglichkeit der Abstraktion über FE lexikalischer Frames im Vordergrund stehen, durch die die KtE dieses KE stets semantisch motiviert werden. In Unterabschnitt 7.3.3 widme ich mich schließlich einer grundsätzlich anderen Möglichkeit, die das KE WEG bzw. 〈WEG〉 betrifft und für die ich in Anlehnung an den Begriff der Makro-Rolle in der Role and Reference Grammar (z.B. Van Valin \& LaPolla 1997: 139-147; Kailuweit 2004) den Begriff des Makro-FE einführen möchte, der als Generalisierung über die bei FrameNet in einem Core Set zusammengefassten FE (Ruppenhofer et al. 2016: 25) zu verstehen ist, zu denen im Falle von Motion die FE Motion.DIRECTION, Motion.DistanCE, Motion.GoAL, Motion.PATH und Motion.SourCE gehören.

\subsubsection{Frame-zu-Frame-Relationen und KtE als Targets lexikalischer Frames}

Als das erste zu benennende und definierende KE der drei untersuchten Konstruktionen sei das KE EREIGNIs betrachtet. Anders als KE wie BEWEGENDES und WEG bzw. 〈WEG〉, deren Benennung und Definition ich in den nächsten beiden Unter- 
abschnitten 7.3.2 und 7.3.3 betrachte, ist die konstruktikographische Beschreibung dieses KE nicht durch eine Generalisierung über FE lexikalischer Frames oder des Konstruktions-Frames möglich. Der Grund dafür ist simpel: Die KtE des KE EREIGNIS dienen als Targets lexikalischer Frames. Sie sind also keine Instanzen von FE, sondern LE, die selbst Frames evozieren. Valenztheoretisch betrachtet sind das KE EREIGNIS und seine KtE „strukturelles Zentrum“ (Willems \& Coene 2006: 240) der Konstruktion. ${ }^{13} \mathrm{Um}$ das KE EREIGNIS nach semantischen Kriterien zu benennen und zu definieren, können also keine FE herangezogen werden, vielmehr müssen die lexikalischen Frames selbst, die durch die KtE dieses KE evoziert werden können, als Grundlage für eine Generalisierung dienen. Eine solche Generalisierung verläuft gewissermaßen über zwei Ebenen: So stellen bereits die lexikalischen Frames, die die KtE dieses KE evozieren, eine Generalisierung dar, da sie über die einzelnen lexikalischen Bedeutungen der LE abstrahieren (vgl. Unterabschnitt 4.1.1 sowie Abbildung 5.3 in Unterabschnitt 5.3.3). Ein lexikalischer Frame entspricht also bereits einer ,semantic generalization across the meanings of the collo-items [= KtE eines KE, A.W.]“ (Herbst 2018b: 12). Ein weiterer Generalisierungsschritt besteht darin, wiederum über diese lexikalischen Frames zu abstrahieren.

Als Methode für eine solche Generalisierung über lexikalische Frames bietet es sich an, Frame-zu-Frame-Relationen heranzuziehen, genauer: ausgehend von einem lexikalischen Frame so weit zu abstrahieren, wie es über Frame-zu-FrameRelationen möglich ist. Das bedeutet, für die einzelnen lexikalischen Frames so weit wie möglich hierarchisch übergeordnete Frames zu finden, im besten Fall den in der jeweiligen Hierarchie höchstgelegenen Frame, der selbst keinen übergeordneten Frame mehr besitzt. ${ }^{14}$ Die diesem Verfahren zugrunde liegende Annahme ist, dass Name und die Definition eines so zu ermittelnden Frames denjenigen des für das zu benennende KE am nächsten kommen. In ähnlicher Weise gehen Perek \& Patten (2019: 374-376) vor, die dafür argumentieren, dass ein Frame, der den in einzelnen Mustern oder Teil-Konstruktionen evozierten Frames übergeordnet ist, als semantische Generalisierung (in etwa: als Konstruktions-Frame der abstrak-

13 Im Kontrast dazu vergeben Ágel \& Höllein (2021: 146-148) auch für Prädikate, also frameevozierende LE, semantische Rollen, ohne zu reflektieren, dass es sich dabei präziser um eine Zuordnung zu Prädikatsklassen (z.B. im Sinne von von Polenz 2008: 159-167) handelt.

14 Eine verwandte (noch vor FrameNet entwickelte) frame-semantische Methode ist die der Hyperonymentypenreduktion (Konerding 1993: 173-181; Ziem 2008: 308-318, 2014b: 264-273): Um den Frame, den eine LE evoziert, zu ermitteln, wird zunächst wörterbuchbasiert das höchstrangige Hyperonym dieser LE bestimmt. Anschließend kann für dieses Hyperonym ein vordefinierter Matrix-Frame (Konerding 1993: 181-201) ausgewählt werden, der die potenziellen FE, die mit der Ausgangs-LE assoziiert werden können, enthält. Das hier vorgeschlagene Verfahren operiert jedoch freilich nicht auf der Ebene von LE, sondern auf derjenigen von Frames. 
teren Konstruktion) gelten kann. Auch sie verwenden dafür den Frame, der als „highest-level semantic generalisation in the network“ (Perek \& Patten 2019: 374) dient. Diese Methode lässt sich, wie ich im Folgenden zeigen möchte, gleichermaßen für die Definition und Benennung eines KE wie EREIGNIS anwenden.

Als Frame-zu-Frame-Relation, die für eine solche Generalisierung verwendet werden kann, kommt in erster Linie die Vererbungsrelation infrage, da sie (wie bereits in den Unterabschnitten 5.4.3 und 6.2.1 erwähnt) „the strongest relation between frames“ (Ruppenhofer et al. 2016: 80) ist. ${ }^{15}$ Ausgehend von den lexikalischen Frames, die von den KtE des KE EREIGNIS evoziert werden, ist nun also zu fragen, welche Frames in der Hierarchie der Vererbungsrelation diesen lexikalischen Frames am weitesten übergeordnet sind. Dabei ist, wie bei dem Konzept der Frame-Nähe (Abschnitt 5.4), eine Abstraktion über mehrere Hierarchieebenen hinweg problemlos möglich. Für alle lexikalischen Frames, die für die drei untersuchten Konstruktionen belegt sind, ist nun die Verteilung der Frames, die ihnen in der Hierarchie der Vererbungsrelation am weitesten übergeordnet sind, zu untersuchen. Die eben aufgestellte Annahme, dass Namen und Definition dieser Frames denjenigen des zu benennenden KE nahekommen sollten, ist methodisch dahingehend zu präzisieren, dass derjenige dieser Frames, der für die drei Konstruktionen am frequentesten ist, am ehesten zur konstruktikographischen Beschreibung des KE EREIGNIS herangezogen werden kann.

Die Daten, die die Grundlage für diese konstruktikographische Analyse bilden, liegen durch die Analysen aus Kapitel 6 bereits größtenteils vor. Schon die Untersuchung der semantischen Motivierungen der einzelnen Strukturelemente der Konstrukte der drei Konstruktionen hat die Verteilung lexikalischer Frames über alle diese Konstrukte hervorgebracht. Bei genauerem Hinsehen wird gar deutlich, dass ein Teil der nötigen Generalisierung bereits vorgenommen wurde: Alle relatierten lexikalischen Frames (Unterabschnitt 5.4.2) sind bereits durch ihre Framezu-Frame-Relationen zu Motion in dieser Hinsicht in den Tabellen 5.12 bis 5.17 in Unterabschnitt 5.4.3 charakterisiert worden. Für sie ist es nur noch nötig, den höchsten zu Motion übergeordneten Frame festzustellen, da sie bereits über eine ganze Bandbreite an Frame-zu-Frame-Relationen auf Motion bezogen werden können. Der höchste zu Motion übergeordnete Frame lässt sich aus Abbildung 5.4 im Eingang zu Abschnitt 5.4 ablesen: Es handelt sich dabei um den Frame Event. Dieser ist Motion direkt übergeordnet, zugleich aber ebenso der in dieser Hierar-

15 Dadurch, dass die Vererbungsrelation als als „,corresponding to is-a in many ontologies“ (Ruppenhofer et al. 2016: 80) gilt, ist sie grundsätzlich mit der semantischen Relation der Hyponymie, wie sie in der Hyperonymentypenreduktion (Konerding 1993: 173-181; Ziem 2008: 308-318, 2014b: 264-273) zum Einsatz kommt, vergleichbar (zur semantischen Relation der Hyponymie vgl. auch Löbner 2015: 232-234). 
chie höchstgelegene Frame, da es keinen Frame gibt, der ihm wiederum in einer Vererbungsrelation übergeordnet wäre. Für alle Konstrukte, die einen relatierten lexikalischen Frame evozieren, ist mit Event also bereits der Frame, der in die Generalisierung zur Benennung und Definition des KE EREIGNIS eingehen kann, festgestellt. ${ }^{16}$

Übrig bleiben nun noch die unrelatierten lexikalischen Frames. Für sie muss in jedem Einzelfall überprüft werden, welcher Frame den in der Vererbungsrelation höchstgelegenen darstellt. Tabelle 7.6 zeigt, für jede der drei untersuchten Konstruktionen getrennt, die Verteilung weiterer höchstrangiger Frames, die auf unrelatierte lexikalische Frames zurückgehen sowie die Anzahl derjenigen Konstrukte (Kx) mit unrelatierten lexikalischen Frames, die ebenso wie relatierte Frames auf den Frame Event zurückzuführen sind. ${ }^{17}$

Diese Gegenüberstellung zeigt deutlich, dass bereits die durch Konstrukte mit relatierten lexikalischen Frames belegte hohe Frequenz des Frames Event eine hinreichende Generalisierung darstellt, die von den Konstrukten mit unrelatierten lexikalischen Frames nicht mehr verändert wird. Es zeigt sich gar, dass die Mehrheit der unrelatierten lexikalischen Frames gleichermaßen auf den Frame Event zurückzuführen ist, weshalb seine Frequenz dadurch insgesamt noch einmal ansteigt. Die Benennung des KE EREIGNIS in Analogie zum Frame Event lässt sich durch diesen Befund empirisch stützen.

Die hohe Frequenz lexikalischer Frames, die auf Event als höchstrangigen Frame zurückzuführen sind, ist als Präferenz im Sinne des gleichnamigen semantischen Parameters für Konstruktionen (Abschnitt 5.3) zu deuten. Konkreter ist für das KE EREIGNIS die Präferenz zu formulieren, als KtE solche LE zu instanziieren, die einen lexikalischen Frame evozieren, der auf Event als höchstrangigen Frame zurückzuführen ist. Als solche sind diese Präferenzen gemäß der in Unterabschnitt 7.1.3 getroffenen Zuordnung semantischer Parameter in die Definition des KE zu integrieren.

16 Man mag an dieser Stelle einwenden, dass auch noch andere Frame-zu-Frame-Relationen als die Vererbungsrelation betrachtet werden könnten und Motion über weitere, noch abstraktere, übergeordnete Frames verfügen könnte. Tatsächlich zeigt sich aber, dass es nur zwei Frames gibt, die Motion in einer anderen Frame-zu-Frame-Relation übergeordnet sind: Motion_scenario in der Perspektive_auf-Relation und Cause_motion in der Kausativ-Relation (vgl. die Tabellen 5.15 und 5.16 in Unterabschnitt 5.4.3). Es steht allerdings intuitiv außer Frage, dass Event einen größeren Abstraktionsgrad als Motion_scenario und Cause_motion aufweisen muss, weswegen dieser Befund an dem Ergebnis der Analyse nichts ändert.

17 Ich kürze die Konstruktionen in Tabelle 7.6 wieder als RBKxn (reflexive Bewegungskonstruktion), RPVKxn (reflexive Partikelverbkonstruktion) und Weg-Kxn (reflexive Weg-Konstruktion) ab. 
Tab. 7.6: Höchstrangige Frames in FrameNet 1.7 in Vererbungsrelation zu den lexikalischen Frames der drei untersuchten Konstruktionen

\begin{tabular}{lrrrrrr}
\hline & \multicolumn{2}{c}{ RBKxn } & \multicolumn{2}{c}{ RPVKxn } & \multicolumn{2}{c}{ Weg-Kxn } \\
Frame & Kx & Anteil & Kx & Anteil & Kx & Anteil \\
\hline Event & 983 & $97,23 \%$ & 821 & $96,59 \%$ & 27 & $100,00 \%$ \\
Scrutiny & 7 & $0,68 \%$ & & & & \\
State & 4 & $0,39 \%$ & & & & \\
Experiencer_focus & 3 & $0,30 \%$ & 4 & $0,47 \%$ & & \\
Impact & 3 & $0,30 \%$ & 4 & $0,47 \%$ & \\
Make_noise & 3 & $0,30 \%$ & 1 & $0,12 \%$ & & \\
Reciprocality & 3 & $0,30 \%$ & 5 & $0,59 \%$ & & \\
Board_vehicle & & & 2 & $0,24 \%$ & & \\
Cogitation & & & 2 & $0,24 \%$ & & \\
Fire_burning & & & 2 & $0,24 \%$ & & \\
Participation & & & 2 & $0,24 \%$ & & \\
Questioning & & & 2 & $0,24 \%$ & & \\
Progression & 2 & $0,20 \%$ & 1 & $0,12 \%$ & & \\
Attributes & & & 1 & $0,12 \%$ & & \\
Change_of_phase & & & 1 & $0,12 \%$ & & \\
Hunting & 1 & $0,10 \%$ & & & & \\
Perception & 1 & $0,10 \%$ & 2 & $0,24 \%$ & & \\
Prevarication & 1 & $0,10 \%$ & & & & \\
\hline Gesamt & 1.011 & $100,00 \%$ & 850 & $100,00 \%$ & 27 & $100,00 \%$ \\
\hline
\end{tabular}

Die Definition des KE kann über diese Präferenzen hinaus potenziell im Rückgriff auf die Definition des höchstrangigen Frames erweitert werden. Für den Fall des KE EREIGNIS und den Frame Event ist dies allerdings trivialer Natur. Die Definition des Frames lautet: „An EvenT takes place at a Place and Time.“ (FrameNet 1.7, Event). Aufgrund der hohen Abstraktheit dieses Frames, der in FrameNet keinen übergeordneten Frame besitzt, ist diese maximal unspezifische Definition allerdings erwartbar und somit von nur geringem Wert. Für Konstruktionen, bei deren Analyse eines KE sich ein anderer (ggf. spezifischer) Frame ergibt, mögen die Konsequenzen, mit denen die Definition des Frames in die Definition des KE einfließen kann, ungleich größer sein.

\subsubsection{Abstraktion über FE lexikalischer Frames}

Anders als das KE EREIGNIS, dessen Benennung und Definition über lexikalische Frames selbst erfolgen kann, werden KE wie BewEgendes und WEG bzw. 〈WEG〉 nicht durch LE instanziiert, die einen lexikalischen Frame evozieren. Wie die Ana- 
lysen in Kapitel 6 gezeigt haben, werden ihre KtE durch FE motiviert, wobei drei Varianten möglich sind: eine einfache Motivierung durch den lexikalischen Frame, eine einfache Motivierung durch den Konstruktions-Frame sowie eine doppelte Motivierung durch beide Frames gemeinsam. Ihre Benennung und Definition kann also nicht, wie bei dem KE EREIGNIS, durch eine Abstraktion über Frames an sich erfolgen, sondern muss bestimmte FE in den Blick nehmen, auf deren Grundlage das KE benannt und definiert werden kann.

Diese Art der Generalisierung ist in der gebrauchsbasierten Konstruktionsgrammatik durchaus bekannt, wenngleich nicht auf der Basis von FrameNetFrames. Schon Goldberg (2002) sieht die semantischen Entsprechung (Argumentrollen) der Strukturelemente von Argumentstruktur-Konstruktionen ${ }^{18}$ als Generalisierungen der semantischen Rollen (Partizipantenrollen) von Verben an: ${ }^{19}$

\footnotetext{
Argument roles capture surface generalizations over individual verbs' participant roles. That is, each distinct sense of a verb is conventionally associated with rich frame semantic meaning that in part specifies certain participant roles: the number and type of slots that are associated with a given sense of a verb. (Goldberg 2002: 342)
}

Auch Herbst (2018b: 11-12) listet aus valenztheoretischer Sicht neben semantischen Merkmalen, Paraphrasen und Listen von Kollexemen (also möglichen KtE) semantische Rollen als eine Option zur Charakterisierung der Komplement-Slots von Argumentstruktur-Konstruktionen auf. Zwar kritisiert er, dass „semantic roles are of little value when it comes to giving an indication of which lexical items occur in a particular valency slot“ (Herbst 2018b: 11), konstruktionssemantisch und konstruktikographisch kann diesem Mangel aber durch einen Zugang zu den annotierten Daten begegnet werden, der in etwa der Auflistung von Kollexemen (im Sinne der Kollostruktionsanalyse von Stefanowitsch \& Gries 2003) bzw. KtE eines KE - einem „collo-profile“ (Herbst 2018b: 12) - nahekommt. Komplementär zu der in Unterabschnitt 7.3.1 diskutierten Generalisierung über die lexikalischen Bedeutungen der KtE eines KE wie EREIGNIS - einer „semantic generalization across the meanings of the collo-items" (Herbst 2018b: 12) - stellt die konstruktikographische Beschreibung von KE auf Basis einer Abstraktion von FE lexikalischer Frames eine „semantic generalization concerning the function of the slot in the construction in terms of an argument role“ (Herbst 2018b: 12) dar.

18 Argumentrollen sind in diesem Sinne durchaus - wenngleich in anderer semantischer Herangehensweise - als Äquivalente zu KE zu verstehen: „The role labels are simply intended as shorthand to capture the semantic properties associated with slots in an argument structure construction.“ (Goldberg 2005b: 224).

19 Auch Dowty (1991: 577) versteht seine Proto-Rollen (Proto-Agens und Proto-Patiens) als Generalisierungen über lexikalische Bedeutungen. 
In den drei von mir untersuchten Konstruktionen ist es in erster Linie des KE Bewegendes, dessen Benennung und Definition nach der Methode der Abstraktion über die FE lexikalischer Frames durchgeführt werden kann. Der Grund dafür: Die FE lexikalischer Frames, die für die Motivierung seiner KtE infrage kommen, sind deutlich zahlreicher als diejenigen, die die KtE des KE WEG bzw. $\langle$ WEG $\rangle$ motivieren, da die Motivierung Letzterer vor allem um die ein Core Set bildenden FE Motion.Source, Motion.PATH, Motion.GoAL und Motion.DIRECTION des Konstruktions-Frames zentriert (Unterabschnitt 7.3.3), aber nicht darauf beschränkt ist (Unterabschnitt 6.4.1). Für die Abstraktion über FE lexikalischer Frames, die die Grundlage für die Benennung und Definition des KE BEwEGENDES bilden, sind grundsätzlich zwei Möglichkeiten denkbar, welche die Herkunft der dafür heranzuziehenden Frames betreffen:

a) eine Abstraktion über die bereits für das KE EREIGNIS analysierten höchstrangigen Frames (Unterabschnitt 7.3.1), die den belegten lexikalischen Frames übergeordnet sind;

b) eine Abstraktion über alle für die Konstruktion belegten lexikalischen Frames selbst, ohne eine vorherige Abstraktion auf die ihnen höchstrangig übergeordneten lexikalischen Frames.

Möglichkeit a besitzt den Vorteil, dass bereits auf die für die Benennung und Definition des KE EREIGNIS erzielten Ergebnisse (Unterabschnitt 7.3.1) zurückgegriffen werden kann, sodass nur eine geringere Anzahl von Types (lexikalischen Frames) analysiert werden muss. Möglichkeit b hingegen lässt potenziell differenziertere Ergebnisse zu, macht aber eine erneute Analyse aller für eine Konstruktion belegten lexikalischen Frames erforderlich. Ich möchte beide Möglichkeiten im Folgenden für die drei untersuchten Konstruktionen miteinander vergleichen und das letztendlich angewandte Verfahren begründen.

Da für Möglichkeit a auf die bereits für die Benennung und Definition des KE EREIGNIS erhobenen Daten zurückgegriffen werden kann, ist sie einfach zu realisieren. Ausgehend von den ermittelten Frames muss nun noch für jeden Frame ermittelt werden, welches FE jeweils für eine potenzielle Motivierung der KtE des KE BEwEGENDES infrage kommt. In den Tabellen 7.7 bis 7.9 sind für die drei Konstruktionen die jeweils höchstrangigen Frames und deren FE, die potenziell zur Motivierung der KtE des KE WEG infrage kommen, dargestellt.

Unschwer zu erkennen ist, dass die hohe Frequenz des Frames Event, die schon zur Benennung und Definition des KE EREIGNIS herangezogen werden konnte, an dieser Stelle keine Abhilfe bietet. Der Grund dafür ist, dass das re- 
Tab. 7.7: Höchstrangige Frames und FE in FrameNet 1.7 zur potenziellen Motivierung der KtE des KE BEWEGENDES der reflexiven Bewegungskonstruktion

\begin{tabular}{llrr}
\hline Frame & FE & Konstrukte & Anteil \\
\hline Event & EVENT & 983 & $97,23 \%$ \\
Scrutiny & COGNIZER & 7 & $0,68 \%$ \\
State & ENTITY & 4 & $0,39 \%$ \\
Experiencer_focus & EXPERIENCER & 3 & $0,30 \%$ \\
Impact & IMPACTOR & 3 & $0,30 \%$ \\
Make_noise & NoISY_EVENT, Sound, Sound_SOURCE & 3 & $0,30 \%$ \\
Reciprocality & PROTAGONIST_1 & 3 & $0,30 \%$ \\
Progression & ENTITY & 2 & $0,20 \%$ \\
Hunting & HUNTER & 1 & $0,10 \%$ \\
Perception & PERCEIVER & 1 & $0,10 \%$ \\
Prevarication & SPEAKER & 1 & $0,10 \%$ \\
\hline Gesamt & & 1.011 & $100,00 \%$ \\
\hline
\end{tabular}

levante FE in FrameNet 1.7 ebenfalls als EvENT bezeichnet ist. ${ }^{20}$ Die Definition dieses FE mit „Name of the event which occurs.“ (FrameNet 1.7, Event) trägt ebenso nichts zur Klärung bei. Da EREIGNIS als Bezeichnung für ein KE bereits vergeben ist, führt diese Methode also nicht zu verwertbaren Ergebnissen.

Möglichkeit $b$ hingegen ist aufgrund der differenzierteren Ergebnisse erfolgversprechender. Sie ist zu realisieren, indem man alle für die drei Konstruktionen belegten lexikalischen Frames daraufhin untersucht, welche FE für die Motivierung der KtE des KE BEWEGENDES infrage kommen, ohne dabei mit der Hilfe von Frame-zu-Frame-Relationen eine Abstraktion auf den jeweils höchstrangigen Frame wie bei Möglichkeit a durchzuführen. Ich beginne hierfür mit der reflexiven Bewegungskonstruktion. In den Tabellen 7.10 und 7.11 sind, geordnet nach relatierten und unrelatierten lexikalischen Frames, alle für diese Konstruktion belegten lexikalischen Frames zusammen mit deren FE, die für die Motivierung der KtE des KE BEWEGENDES infrage kommen, zusammengestellt.

Die Ergebnisse zu den unrelatierten lexikalischen Frames fallen sehr differenziert aus: Die Bandbreite an relevanten FE ist, wie Tabelle 7.11 zeigt, äußerst groß. Dies ist zu erwarten, da die unrelatierten lexikalischen Frames nicht in FrameNähe zu einem gemeinsamen Konstruktions-Frame stehen und daher Ähnlich-

20 Diese Praxis von FrameNet ist Anlass zur Kritik, scheint es doch angebrachter, eine Dopplung von Bezeichnungen zwischen dem Frame-Namen und dem Namen eines FE zu vermeiden. Andererseits ist nachvollziehbar, dass es für solch abstrakte Frames wie Event grundsätzlich schwierig sein dürfte, geeignete Bezeichnungen zu finden. Gleiches gilt für die Definitionen solcher Frames und ihrer FE. 
Tab. 7.8: Höchstrangige Frames und FE in FrameNet 1.7 zur potenziellen Motivierung der KtE des KE BeWEgendes der reflexiven Partikelverbkonstruktion

\begin{tabular}{llrr}
\hline Frame & FE & Konstrukte & Anteil \\
\hline Event & EVENT & 821 & $96,59 \%$ \\
Reciprocality & PROTAGONIST_1 & 5 & $0,59 \%$ \\
Experiencer_focus & EXPERIENCER & 4 & $0,47 \%$ \\
Impact & IMPACTOR & 4 & $0,47 \%$ \\
Board_vehicle & TRAVELLER & 2 & $0,24 \%$ \\
Cogitation & COGNIZER & 2 & $0,24 \%$ \\
Fire_burning & FIRE & 2 & $0,24 \%$ \\
Participation & PARTICIPANT_1 & 2 & $0,24 \%$ \\
Perception & PERCEIVER & 2 & $0,24 \%$ \\
Questioning & SPEAKER & 2 & $0,24 \%$ \\
Attributes & ENTITY & 1 & $0,12 \%$ \\
Change_of_phase & PATIENT & 1 & $0,12 \%$ \\
Make_noise & NoISY_EVENT, SOUND, SOUND_SOURCE & 1 & $0,12 \%$ \\
Progression & ENTITY & 1 & $0,12 \%$ \\
\hline Gesamt & & 850 & $100,00 \%$ \\
\hline
\end{tabular}

Tab. 7.9: Höchstrangige Frames und FE in FrameNet 1.7 zur potenziellen Motivierung der KtE des KE BEWEGENDES der reflexiven Weg-Konstruktion

\begin{tabular}{llrr}
\hline Frame & FE & Konstrukte & Anteil \\
\hline Event & EVENT & 27 & $100,00 \%$ \\
\hline Gesamt & & 27 & $100,00 \%$ \\
\hline
\end{tabular}

keiten zwischen ihren FE nicht durch entsprechende Frame-zu-Frame-Relationen motiviert sein können (vgl. auch die Kritik an Goldbergs Semantic Coherence Principle in Unterabschnitt 6.2.4). Für die relatierten lexikalischen Frames (Tabelle 7.10) gestaltet sich dies anders. Klar zu erkennen ist, dass zwei FE besonders hervorstechen: AGENT und THEME. Sie liegen quantitativ so nahe beieinander, dass keine deutliche Präferenz zugunsten des einen oder des anderen beobachtet werden kann. Zwei Faktoren können jedoch bei der Entscheidung helfen, welches FE eher als Grundlage für die Definition des KE BEWEGENDEs herangezogen werden kann. 
Tab. 7.10: Relatierte lexikalische Frames und FE in FrameNet 1.7 zur potenziellen Motivierung der KtE des KE BEWEGENDES der reflexiven Bewegungskonstruktion

\begin{tabular}{llrr}
\hline Frame & FE & Konstrukte & Anteil \\
\hline Body_movement & AGENT & 246 & $34,55 \%$ \\
Self_motion & SELF_MOVER & 159 & $22,33 \%$ \\
Motion & THEME & 105 & $14,75 \%$ \\
Cause_motion & AGENT & 77 & $10,81 \%$ \\
Change_direction & THEME & 43 & $6,04 \%$ \\
Bringing & AGENT & 17 & $2,39 \%$ \\
Fluidic_motion & FLUID & 17 & $2,39 \%$ \\
Placing & AGENT & 14 & $1,97 \%$ \\
Motion_directional & THEME & 11 & $1,54 \%$ \\
Evading & EVADER & 7 & $0,98 \%$ \\
Departing & THEME & 6 & $0,84 \%$ \\
Fleeing & SELF_MOVER & 5 & $0,70 \%$ \\
Mass_motion & MASS_THEME & 2 & $0,28 \%$ \\
Making_faces & AGENT & 1 & $0,14 \%$ \\
Ride_vehicle & THEME & 1 & $0,14 \%$ \\
Undressing & WEARER & 1 & $0,14 \%$ \\
\hline Gesamt & & 712 & $100,00 \%$ \\
\hline
\end{tabular}

- Der Grad der Frame-Nähe: Lexikalische Frames mit einer höheren FrameNähe zum Konstruktions-Frame sind als relevanter einzustufen.

- Die Art der Frame-zu-Frame-Relation: Lexikalische Frames, die z.B. in Vererbungsrelation zum Konstruktions-Frame stehen, sind als relevanter einzustufen als solche, die z.B. in der Benutzt- oder Siehe_auch-Relation zu ihm stehen.

Interpretiert man die Ergebnisse in Tabelle 7.10 entsprechend, so ist festzuhalten, dass ein Frame wie Self_motion, der in einer Frame-Nähe von +1 in Vererbungsrelation zum Konstruktions-Frame steht, höher zu bewerten ist als der Frame Body_movement, der zwar ebenfalls in einer Frame-Nähe von +1 , dafür aber in der Benutzt-Relation zu Motion steht. Dies liegt allein daran, dass die Vererbungsrelation die „strongest relation between frames, corresponding to is-a in many ontologies“ (Ruppenhofer et al. 2016: 80) darstellt. Entsprechend wie Self_motion zu bewerten sind die Frames Fluidic_motion, Motion_directional und Mass_motion. Bleibt man bei der Vererbungsrelation und wechselt zu einer Frame-Nähe von +2 , kommt etwa noch Fleeing hinzu. Die FE dieser Frames, die für die Motivierung der KtE des KE BEwEGENDES infrage kommen, teilen eine Gemeinsamkeit mit dem FE Motion.THEME des Konstruktions-Frames, was bereits an ihren Namen 
Tab. 7.11: Unrelatierte lexikalische Frames und FE zur potenziellen Motivierung der KtE des KE BEWEGENDES der reflexiven Bewegungskonstruktion

\begin{tabular}{|c|c|c|c|}
\hline Frame & $\mathrm{FE}$ & Kx & Anteil \\
\hline Cause_to_move_in_place & AgENT & 118 & $39,46 \%$ \\
\hline Cause_to_amalgamate & AgENT & 30 & $10,03 \%$ \\
\hline Manipulation & AgENT & 20 & $6,69 \%$ \\
\hline Daring & AgENT & 18 & $6,02 \%$ \\
\hline Seeking & COGNIZER_AGENT & 14 & $4,68 \%$ \\
\hline Work & AgENT & 13 & $4,35 \%$ \\
\hline Cause_to_experience & AgENT & 11 & $3,68 \%$ \\
\hline Hostile_encounter & SIDE_1 & 11 & $3,68 \%$ \\
\hline Ingestion & INGESTOR & 10 & $3,34 \%$ \\
\hline Scouring & SEARCHER & 7 & $2,34 \%$ \\
\hline Rescuing & AGENT & 5 & $1,67 \%$ \\
\hline Chatting & INTERLOCUTOR_1 & 3 & $1,00 \%$ \\
\hline Desiring & EXPERIENCER & 3 & $1,00 \%$ \\
\hline Emptying & AgENT & 3 & $1,00 \%$ \\
\hline Impact & IMPACTOR & 3 & $1,00 \%$ \\
\hline Make_noise & NOISY_EVENT, SOUND, SOUND_SOURCE & 3 & $1,00 \%$ \\
\hline Others_situation_as_stimulus & EXPERIENCER & 3 & $1,00 \%$ \\
\hline Reshaping & DEFORMER & 3 & $1,00 \%$ \\
\hline Attaching & AgENT & 2 & $0,67 \%$ \\
\hline Filling & AgENT & 2 & $0,67 \%$ \\
\hline Giving & DONOR & 2 & $0,67 \%$ \\
\hline Progression & ENTITY & 2 & $0,67 \%$ \\
\hline Smuggling & Perpetrator & 2 & $0,67 \%$ \\
\hline Assistance & HELPER & 1 & $0,33 \%$ \\
\hline Burying & AgENT & 1 & $0,33 \%$ \\
\hline Cause_bodily_experience & AgENT & 1 & $0,33 \%$ \\
\hline Cause_harm & AgENT & 1 & $0,33 \%$ \\
\hline Dead_or_alive & PROTAGONIST & 1 & $0,33 \%$ \\
\hline Grinding & GRINDER & 1 & $0,33 \%$ \\
\hline Hunting & HUNTER & 1 & $0,33 \%$ \\
\hline Manipulate_into_doing & MANIPULATOR & 1 & $0,33 \%$ \\
\hline Perception_experience & PERCEIVER_PASSIVE & 1 & $0,33 \%$ \\
\hline Prevarication & SPEAKER & 1 & $0,33 \%$ \\
\hline Shopping & SHOPPER & 1 & $0,33 \%$ \\
\hline$\overline{\text { Gesamt }}$ & & 299 & $\overline{100,00 \%}$ \\
\hline
\end{tabular}


Self_motion.SELF_MOVER, Fluidic_motion.FLUID, Motion_directional.THEME, Mass_motion.MASS_THEME und Fleeing.SELF_MOVER zu erkennen ist (vgl. dazu auch Unterabschnitt 6.2.1). Hinzu kommt noch der Konstruktions-Frame Motion selbst mit dem FE Theme. Dessen Definition kann stellvertretend für die zuvor genannten FE gelten. Sie lautet: „The THEME is the entity that changes location. Note that it is not necessarily a self-mover.“ (FrameNet 1.7, Motion). Berücksichtigt man diese Definition, so lässt sich Theme vor dem Hintergrund, dass alle relevanten Frames in Frame-Nähe zu Motion stehen, mit Bewegendes übersetzen. ${ }^{21}$ Diese Beobachtung ist die wesentliche Motivation dafür, das relevante KE der reflexiven Bewegungskonstruktion entsprechend $\mathrm{zu}$ benennen und $\mathrm{zu}$ definieren.

Zunächst sei aber noch überprüft, ob diese für die reflexive Bewegungskonstruktion geltenden Beobachtungen auch auf die anderen beiden untersuchten Konstruktionen zutreffen. Für die reflexive Partikelverbkonstruktion, deren Daten in den Tabellen 7.12 und 7.13 dargestellt sind, ergibt sich ein ähnliches Bild. Hinsichtlich der unrelatierten lexikalischen Frames (Tabelle 7.13) liegt eine noch größere Bandbreite an FE als für die reflexive Bewegungskonstruktion vor, da die Anzahl der Types unrelatierter lexikalischer Frames für die reflexive Partikelverbkonstruktion höher liegt (vgl. die Tabellen 7.18 und 7.19 in Unterabschnitt 7.5.2). Aufgrund dieser Bandbreite scheint es auch hier angebrachter, für die Benennung und Definition des relevanten KE die Daten der relatierten lexikalischen Frames heranzuziehen. ${ }^{22}$ Deren Verteilung in Tabelle 7.12 zeigt eine leichte Präferenz des FE AGENT gegenüber des FE THEME, anders als dies bei der reflexiven Bewegungskonstruktion der Fall war. Wendet man aber auch hier die beiden eben erwähnten Kriterien des Grades der Frame-Nähe sowie der Art der Frame-zu-Frame-Relation an, zeigt sich gleichermaßen eine Präferenz für THEME sowie das verwandte FE Self_motion.SELF_MOVER. Ich übertrage deshalb die Analyse der reflexiven Bewegungskonstruktion auf die reflexive Partikelverbkonstruktion und benenne und definiere auch ihr KE BEwEGENDEs in Anlehnung an das FE THEME.

Zuletzt sei noch ein Blick auf die reflexive Weg-Konstruktion geworfen. In Tabelle 7.14 sind die Daten für den einzig belegten relatierten sowie den einzig beleg-

21 Die Bezeichnung Bewegendes kann abkürzend für die näher an der Definition des FE Motion.THEME stehende Bezeichnung Bewegende Entität dienen. Um sie nicht unnötig komplex werden zu lassen, bietet sich also Bewegendes eher an.

22 Die enorm hohe Token-Frequenz des unrelatierten lexikalischen Frames Cause_to_move_ in_place in Tabelle 7.13 ist auf den Status des KE 〈WEG〉 als Nicht-Kern-KE zurückzuführen, das dafür sorgt, dass eine große Anzahl von Konstrukten vollständig durch FE dieses lexikalischen Frames motiviert wird, obwohl es sich dabei um einen unrelatierten Frame handelt (vgl. dazu Unterabschnitt 6.4.3). Ich gehe deshalb davon aus, dass die hohe Token-Anzahl für diesen Frame keine Auswirkung auf die Benennung und Definition des KE BEWEgEndes haben muss. 
Tab. 7.12: Relatierte lexikalische Frames und FE in FrameNet 1.7 zur potenziellen Motivierung der KtE des KE BeWEgendes der reflexiven Partikelverbkonstruktion

\begin{tabular}{llrr}
\hline Frame & FE & Konstrukte & Anteil \\
\hline Body_movement & AGENT & 118 & $38,82 \%$ \\
Self_motion & SELF_MOVER & 56 & $18,42 \%$ \\
Cause_motion & AGENT & 53 & $17,43 \%$ \\
Motion & THEME & 24 & $7,89 \%$ \\
Change_direction & THEME & 17 & $5,59 \%$ \\
Motion_directional & THEME & 15 & $4,93 \%$ \\
Placing & AGENT & 11 & $3,62 \%$ \\
Bringing & AGENT & 8 & $2,63 \%$ \\
Excreting & EXCRETER & 1 & $0,33 \%$ \\
Operate_vehicle & DRIVER & 1 & $0,33 \%$ \\
\hline Gesamt & & 304 & $100,00 \%$ \\
\hline
\end{tabular}

ten unrelatierten lexikalischen Frame für diese Konstruktion zusammengefasst. Da der relatierte Frame Motion hier ohnehin die Mehrheit der Konstrukte repräsentiert, ist es für diese Konstruktion, wie auch für die reflexive Bewegungskonstruktion und die reflexive Partikelverbkonstruktion, ebenso angebracht, das KE BEWEGENDES auf Basis des FE Motion.Theme zu benennen und zu definieren.

Für die Benennung und Definition des KE BEWEGENDES aller drei untersuchten Konstruktionen stellt sich somit die direkte Abstraktion über FE lexikalischer Frames, ohne diese auf jeweils höchstrangige Frames, wie bei der Benennung und Definition des KE EREIGNIS (Unterabschnitt 7.3.1), zu abstrahieren, als praktikabler heraus. Die bisher noch fehlende Begründung der Benennung des KE BEwEGENDES ist über diese Methode zu leisten. Sowohl die Benennung als auch die Definition des KE orientieren sich prototypisch an dem FE Motion.THEME, das stellvertretend für verwandte FE in anderen relatierten lexikalischen Frames diese Rolle übernimmt. Die hier vorgestellte Analyse zeigt aber zugleich, dass rein quantitative Auswertungen, wie sie in den drei Tabellen 7.10 bis 7.14 zusammengefasst sind, nicht immer eine verlässliche Datengrundlage bilden, da sie nicht zwangsläufig $\mathrm{zu}$ einem intuitiv passenden Ergebnis führen. So müssen die jeweils frequentesten lexikalischen Frames und deren FE, die für die Motivierung der KtE des KE BEWEGENDES infrage kommen, nicht mit einem erwarteten Ergebnis für die Grundlage von dessen Benennung und Definition übereinstimmen. Ob dies statistisch zufällig ist oder ob die Erwartungen tatsächlich revidiert werden müssen, kann aufgrund der vergleichsweise geringen Datenmenge abschließend nicht entschieden werden. Hier sind weitere Untersuchungen, insbesondere mit größeren Mengen an konstruktionssemantisch annotierten Konstrukten, nötig. 
Tab. 7.13: Unrelatierte lexikalische Frames und FE in FrameNet 1.7 zur potenziellen Motivierung der KtE des KE BewEgendes der reflexiven Partikelverbkonstruktion

\begin{tabular}{|c|c|c|c|}
\hline Frame & $\mathrm{FE}$ & Kx & Anteil \\
\hline Cause_to_move_in_place & AgENT & 411 & $75,27 \%$ \\
\hline Daring & AgENT & 24 & $4,40 \%$ \\
\hline Hostile_encounter & SIDE_1 & 13 & $2,38 \%$ \\
\hline Work & AgENT & 11 & $2,01 \%$ \\
\hline Seeking & COGNIZER_AGENT & 10 & $1,83 \%$ \\
\hline Cause_to_experience & AgENT & 7 & $1,28 \%$ \\
\hline Manipulation & AgENT & 6 & $1,10 \%$ \\
\hline Cause_to_amalgamate & AgENT & 5 & $0,92 \%$ \\
\hline Chatting & INTERLOCUTOR_1 & 5 & $0,92 \%$ \\
\hline Attaching & AgENT & 4 & $0,73 \%$ \\
\hline Filling & AgENT & 4 & $0,73 \%$ \\
\hline Impact & IMPACTOR & 4 & $0,73 \%$ \\
\hline Ingestion & INGESTOR & 4 & $0,73 \%$ \\
\hline Shopping & SHOPPER & 4 & $0,73 \%$ \\
\hline Processing_materials & AgENT & 3 & $0,55 \%$ \\
\hline Board_vehicle & TRAVELLER & 2 & $0,37 \%$ \\
\hline Burying & AgENT & 2 & $0,37 \%$ \\
\hline Cogitation & COGNIZER & 2 & $0,37 \%$ \\
\hline Desiring & EXPERIENCER & 2 & $0,37 \%$ \\
\hline Experiencer_focus & EXPERIENCER & 2 & $0,37 \%$ \\
\hline Fire_burning & FIRE & 2 & $0,37 \%$ \\
\hline Manipulate_into_doing & MANIPULATOR & 2 & $0,37 \%$ \\
\hline Participation & PARTICIPANT_1 & 2 & $0,37 \%$ \\
\hline Questioning & SPEAKER & 2 & $0,37 \%$ \\
\hline Assistance & HELPER & 1 & $0,18 \%$ \\
\hline Attack & ASSAILANT & 1 & $0,18 \%$ \\
\hline Becoming_aware & COGNIZER & 1 & $0,18 \%$ \\
\hline Change_of_phase & Patient & 1 & $0,18 \%$ \\
\hline Change_posture & PROTAGONIST & 1 & $0,18 \%$ \\
\hline Emptying & AgENT & 1 & $0,18 \%$ \\
\hline Grinding & GRINDER & 1 & $0,18 \%$ \\
\hline Make_noise & NOISY_EVENT, SOUND, SOUND_SOURCE & 1 & $0,18 \%$ \\
\hline Perception_experience & PERCEIVER_PASSIVE & 1 & $0,18 \%$ \\
\hline Progression & ENTITY & 1 & $0,18 \%$ \\
\hline Renting & LESSEE & 1 & $0,18 \%$ \\
\hline Rescuing & AgENT & 1 & $0,18 \%$ \\
\hline Temperature & ENTITY & 1 & $0,18 \%$ \\
\hline Gesamt & & 546 & $100,00 \%$ \\
\hline
\end{tabular}


Tab. 7.14: Lexikalische Frames und FE in FrameNet 1.7 zur potenziellen Motivierung der KtE des KE BEWEGENDES der reflexiven Weg-Konstruktion

\begin{tabular}{llrr}
\hline Frame & FE & Konstrukte & Anteil \\
\hline Motion (relatiert) & THEME & 26 & $96,30 \%$ \\
Cutting (unrelatiert) & AGENT & 1 & $3,70 \%$ \\
\hline Gesamt & & 27 & $100,00 \%$ \\
\hline
\end{tabular}

Gleichzeitig zeigt sich, dass auch die FrameNet-Daten nicht immer zuverlässig und für den Zweck der Benennung und Definition von KE treffend sein müssen. Ein Beispiel dafür ist die Bezeichnung Theme, die für sich genommen zu unspezifisch ist, um die semantischen Eigenschaften der Konstruktion in der Benennung des betreffenden KE zu kondensieren. ${ }^{23}$ Treffender ist hier schon die Bezeichnung Self_mover, die das äquivalente FE des Frames Self_motion abbildet, wobei hier wiederum die Einschränkung auf ein „living being which moves under its own power“" (FrameNet 1.7, Self_motion) zu restriktiv ist, um für alle Konstrukte der Konstruktion (auch solche, in denen das KtE von BEWEGENDES auf ein abstraktes, unbelebtes Referenzobjekt referiert) gelten zu können (dazu weiterhin Abschnitt 8.5).

Diese zwei Aspekte zeigen, dass bei der Benennung und Definition von KE methodisch nach wie vor auf eine Mischung aus gebrauchsbasierter (im Idealfall: statistisch belastbarer) Evidenz und linguistischer Intuition zurückgegriffen werden muss (vgl. allgemein dazu Sinclair 1991: 39; Boas 2003a: 11-18; Ziem \& Lasch 2013: 68; Hilpert 2019: 20). Beide schließen sich keineswegs gegenseitig aus: „[I]ntuitions are needed to interpret data, but should not be used to create data." (Hanks 2013: 361).

Rückblickend auf die hier vorgestellte Methode als solche ist das KE BEwEGENDES indes das erste KE der drei untersuchten Konstruktionen, das über die Abstraktion von FE benannt und definiert werden kann. Ein weiteres KE, bei dem dies ebenso möglich und nötig ist, ist das KE WEG bzW. 〈WEG〉.

23 Im FrameNet des Deutschen wird Theme i.d.R. mit Objekt übersetzt, etwa im Frame Bewegung_verursachen, dem deutschen Äquivalent von Cause_motion (vgl. seine FE unter https://gsw.phil.hhu.de/framenet/frame?id=1106\&s=2, zuletzt abgerufen am 07.09.2021). Die Bezeichnung Objekt ist konstruktionssemantisch für das Deutsche als Metasprache aufgrund der Homonymie zur gleichnamigen grammatischen Funktion vermutlich noch problematischer als Theme im Englischen. 


\subsubsection{Makro-FE und Core Sets}

Während das KE EREIGNIS noch über die Abstraktion über lexikalische Frames an sich definiert werden konnte (Unterabschnitt 7.3.1), mussten, wie soeben in Unterabschnitt 7.3.2 gezeigt, für das KE BEWEGENDEs bestimmte FE dieser Frames herangezogen werden. Der Grund dafür liegt darin, dass dieses KE nicht, wie EREIGNIS, primär durch frame-evozierende LE instanziiert wird (vgl. aber Unterabschnitt 8.3.2 für eine differenzierte Betrachtung), sondern dass es durch KtE instanziiert wird, die durch FE der betreffenden lexikalischen Frames motiviert werden. Gleiches gilt nun ebenso für das KE WEG bzw. 〈WEG $\rangle$. Mit einem Unterschied: Die Bandbreite der FE, die die KtE des KE WEG bzw. 〈WEG〉 motivieren, ist prinzipiell eingeschränkter als diejenige, die die KtE des KE BEWEGENDES motivieren. Sie stellt eine eigene frame-semantische Analysekategorie dar, die ich in diesem Unterabschnitt betrachten und für die Benennung und Definition des KE WEG bzw. 〈WEG〉 nutzbar machen möchte.

In Unterabschnitt 6.4.1 habe ich dafür argumentiert, bestimmte FE, die gemeinsam als mehrere KtE instanziiert werden, als Instanzen ein und desselben KE aufzufassen. Für Motion als Konstruktions-Frame der reflexiven Bewegungskonstruktion, der reflexiven Partikelverbkonstruktion und der reflexiven WegKonstruktion sowie zahlreiche von ihm ausgehende relatierte lexikalischen Frames (z.B. Self_motion und Fleeing) betrifft dies die FE Source, PATH, GoAL, DiRECTION und DisTANCE. ${ }^{24}$

Die FE, die als die KtE des KE WEG bzw. 〈WEG〉 infrage kommen, können in unterschiedlichen Kombinationen auftreten. Wird das KE lediglich einfach (also nicht mehrfach, wie in Unterabschnitt 6.4.1 gezeigt) durch ein einziges KtE instanziiert, treten sie einzeln auf, wie es die Belege in (16) illustrieren. ${ }^{25}$

(16) a. Ringeln sich etwa nicht ein paar Schlangen um sie herum im Sand oder in ihrem Nacken, [Motion schlängeln] sich herab [Source von ihrer Brust]. (Venske, Regula: Marthes Vision, Frankfurt am Main: Eichborn Verlag 2006, S. 128)

b. Es war ein wüster Abend, bei dem viel geschrien und grell gelacht wurde; die Darsteller [Hunting jagten] einander [über die Bühne ${ }^{\text {РАTH }}$ ], es knallte an allen Ecken und Enden, so daß man den Text kaum verstand. (Schmitter, Elke: Frau Sartoris, Berlin: BvT 2000[2002], S. 87)

24 Die hier nicht verwendete Doppelnotation (z.B. Motion.SouRCE) soll darauf hinweisen, dass diese FE nicht spezifisch für Motion sind, sondern von zahlreichen weiteren Frames, insbesondere zu ihm relatierten Frames, geteilt werden.

25 Ein FE Distance (z.B. Motion.Distance) ist in meinen Daten nicht belegt. 
c. Am 10. Oktober [Motion begab] sich TSVANGIRAI freiwillig [GoAL zu einem polizeilichen Verhör in Harare]. (Archiv der Gegenwart, 2001 [2000])

d. Er schaute sich noch ein wenig um und begann, sich wieder [in Richtung Ausgang Direction] vorzu[work arbeiten]. (Suter, Martin: Lila, Lila, Zürich: Diogenes 2004, S. 344)

Neben diesem einzelnen Auftreten der entsprechenden FE erscheinen sie, wenn es sich um eine mehrfache Instanziierung des KE WEG bzw. 〈WEG〉 handelt (Unterabschnitt 6.4.1), in unterschiedlichen Kombinationen. Die Belege in (17) sind Beispiele für die Kombination aus den FE SourcE und GoAL.

(17) a. HASSAN [Motion begab] sich Mitte Oktober in Begleitung einer tausend Mann starken bewaffneten Eskorte [Source von Djibouti aus] [GoAL nach Mogadischu], um seine Macht von dort aus auszuüben. (Archiv der Gegenwart, 2001 [2000])

b. Menschenaffen [self_motion hangeln] sich noch heute [Source von Ast] [GoAL $\mathrm{zu}$ Ast], unter elegantem Einsatz von Hand und Fuß. (Die Zeit, 23.03.2000, Nr. 13)

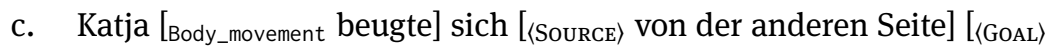
über ihren Bruder] und legte die Hand auf seine Stirn, als sei sie die Mutter. (Franck, Julia: Lagerfeuer, Köln: DuMont Literatur und Kunst Verlag 2003, S. 163)

Die Belege in (18) zeigen die Kombination aus PATH und GoAL.

(18) a. Und wenn die Jungs nach getaner Tat in den Armen der Liebsten schlummern, [smuggling schmuggelt] sich Rudi mittels Dollars und Autogrammfußbällen [РATH am Endlosstau von Brest vorbei ${ }^{\text {PATH }}$ [ [GOAL nach Polen Goal] rein. (Die Zeit, 24.02.2000, Nr. 9)

b. Sie hatte den Damm eines Speichersees durchbrochen, [Fluidic_motion ergoss] sich [РATH durch den Feldkanal] [GoAL in die Lapus], von der Lapus in die Somes und von der Somes jenseits der ungarischen Grenze in die Theiß. (Die Zeit, 24.02.2000, Nr. 9)

Werden alle drei der bisher erwähnten FE gemeinsam in einem Konstrukt instanziiert, entsteht die Kombination Source, PATH und GoAL - verstanden als „maximal windowing over the whole of the conceptually complete path“ (Talmy 1996: 244) -, die in (19) zu sehen ist.

(19) Ein Drahtseil ist über den Fluß gespannt, mit einer beweglichen Winde ist das Fährboot daran festgetäut, und während die gewaltige Strömung 
auf das querstehende Ruder drückt, [Motion bewegen] sich Boot und Winde seitlich [РATH am Seil entlang] [Source von einem Ufer] [GoAL zum andern]. (Düffel, John von: Vom Wasser, München: dtv 2006, S. 192)

Weitere Kombinationen sind möglich, wenn das FE DiRECTION hinzukommt. So findet sich in (20) die Kombination aus SOURCE, GoAL und Direction.

(20) Schiller war kein Autor, der von innen kommt, er [Motion bewegte] sich [Direction in der Gegenrichtung], [Source von außen] [GoaL nach innen]. (Safranski, Rüdiger: Friedrich Schiller, München Wien: Carl Hanser 2004, S. 118)

Es ist unschwer zu erkennen, dass diese FE nicht nur gemeinsam haben, dass sie für die semantische Motivierung von KtE ein und desselben KE infrage kommen. Sie eint darüber hinaus ein semantischer Aspekt: Sie dienen allesamt dazu, den Verlauf der ,Bewegung' zu charakterisieren, die das Referenzobjekt des KtE des KE BEWEGENDES ausführt. FrameNet erfasst diese Zusammengehörigkeit von FE unter dem Begriff Core Set oder Coreness Set.

In our annotation practice, we often find that some groups of FEs seem to act like sets, in that the presence of any member of the set is sufficient to satisfy a semantic valence of the predicator. We refer to such a group of FEs as a coreness set, or CoreSet. For instance, SouRCE, PATH, and GoAL are core FEs in the various motion frames in the database. However, although possible, it is not necessary, and in fact unusual, for all three FEs to co-occur, [...]. (Ruppenhofer et al. 2016: 25, Hervorhebung im Original in Fettdruck)

Obwohl es sich bei den genannten FE von Motion allesamt um Kern-FE handelt, ${ }^{26}$ wird das Fehlen eines oder mehrerer FE in einer Instanz, wie Ruppenhofer et al. (2016: 25-26) weiter festhalten, nicht als Null-Instanziierung markiert. Ruppenhofer \& Michaelis (2014: 63-64) bezeichnen dies stattdessen als einen Fall von Non-Instanziierung. Da eine (nahezu) vollständige Instanziierung des gesamten Core Set, wie sie sich etwa in (19) andeutet, allerdings selten zu finden ist, ist eine solche Non-Instanziierung eines oder mehrerer FE eines Core Set der Regelfall.

Meine in Unterabschnitt 6.4.1 vertretene Annahme, dass die durch FE, die zu einem Core Set gehören, instanziierten KtE - treten sie nun einzeln oder in Kombination auf - als Instanzen ein und desselben KE zu werten sind, bietet die Mög-

26 Dies gilt allerdings nicht für alle zu Motion relatierten Frames. So variiert der Status der beteiligten FE als Kern- oder Nicht-Kern-FE, wenn wie etwa in Body_movement die FE 〈SoURCE), 〈РATH und $\langle$ GOAL $\rangle$ als Nicht-Kern-FE ausgezeichnet sind. Sie sind dort somit nicht als Core Set ausgewiesen, was ich für einen solchen Einzelfall ignoriere, da nicht klar ist, ob diese Feststellung seitens FrameNet auf einer empirischen Beobachtung beruht oder ein Fehler in FrameNet 1.7 ist. 
lichkeit, das betreffende KE, für die drei untersuchten Konstruktionen also WEG bzw. 〈WEG〉, auf der Grundlage des entsprechenden Core Set zu benennen und zu definieren. Die Wahl der Bezeichnung Weg als Oberbegriff für das gesamte Core Set steht im Einklang nicht nur mit der oben zitierten Praxis von Talmy (1996: 244), sondern ebenso mit dem explizit für die way-Konstruktion bzw. die reflexive Bewegungskonstruktion gewählten Vorgehen von Goldberg (1995: 207) und Oya (1999: 360). ${ }^{27}$ Aus der Instanziierung von durch die ein Core Set bildenden FE motivierten KtE lässt sich also die konstruktikographische Beschreibung des zugrunde liegenden KE zu generalisieren. Die Benennung des KE WEG bzw. 〈WEG〉 ist eine Abstraktion über die FE, die die möglichen KtE dieses KE motivieren.

Dieser Arbeitsschritt der Generalisierung ist ein genuin konstruktionssemantischer und konstruktikographischer. Der Grund dafür ist, dass FrameNet selbst diese Generalisierung nicht vornimmt. Somit fehlt ein entscheidender Schritt, der von FrameNet nicht systematisch vollzogen wird: Die Core Sets erhalten üblicherweise keine eigenständigen Benennungen und Definitionen. Am Beispiel der genannten FE von Motion und seiner untergeordneten Frames heißt es allerdings bei Ruppenhofer et al. (2016: 26): „Source, Path, and Goal, for instance, are clearly related via a notion that we might call 'full path'.“

Damit ist nun deutlich, woher das KE WEG, dessen KtE durch eines der FE in dem genannten Core Set motiviert werden kann, seinen Namen bezieht. Alle fünf FE dienen zur Charakterisierung des ,Weges', auf dem sich das Referenzobjekt des KtE des KE BEwEGENDES ,bewegt'. Möglich sind Charakterisierungen des ,Weges‘ an sich (РATH), von dessen ,Ursprung، (SourCE), ,Endpunkt' (GOAL), ,Richtung‘ (Direction) oder dem ,Abstand` zwischen ,Ursprung` und ,Endpunkt‘ (DisTANCE).

Terminologisch lässt sich die Zusammenfassung von $\mathrm{FE}$ zu einem Core Set, darauf weisen Ruppenhofer \& Michaelis (2014: 63-64) implizit hin, als MakroRolle im Sinne der Role and Reference Grammar (z.B. Van Valin \& LaPolla 1997: 139-147; Kailuweit 2004: 96-101) verstehen..$^{28}$ In Anlehnung an diese Terminolo-

27 Vgl. auch Ungerer (2017: 4), der ein „SOURCE-PATH-GOAL schema (henceforth simply PATH schema)“ annimmt. Vgl. auch das Vorgehen von Rohde (2001: 15, 38, 289-294).

28 Dowty (1991: 598-599) weist darauf hin, dass seine Proto-Rollen (Proto-Agens und ProtoPatiens) zu Makro-Rollen vergleichbar sind, mit dem Unterschied, dass Letztere nicht prototypisch, sondern durch schärfere Grenzen definiert sind. Aus frame-semantischer Sicht stellt dies freilich kein Problem dar, da die Anzahl der FE nicht (wie in einem Proto-Rollen-Ansatz) radikal eingeschränkt werden muss. Ferner ist zu berücksichtigen, dass die Konzepte der Makro- und Proto-Rolen vorranging auf ,Kern'-Argumente, die Subjekt- und (direkte) Objekt-Position besetzen, ausgerichtet ist. Über den Makro- oder Proto-Rollen-Status von PP, die die Instanziierungen des KE WEG bilden, wird nicht reflektiert. 
gie möchte ich analog von einem Makro-FE sprechen, das die FE eines Core Set unter sich vereint. ${ }^{29}$

Eine ähnliche Idee formuliert bereits Barsalou (1992b: 34-35) unter dem Begriff der Attribut-Systematizität (vgl. zuvor Barsalou \& Billman 1989: 153-155): Bestimmte $\mathrm{FE},{ }^{30}$ die besonders häufig kookkurrieren, bilden einen Frame-Kern aus (zum Begriff des Frame-Kerns vgl. auch Busse 2012: 563-565, 570-572). Ein solcher Frame-Kern unterscheidet sich von einem Core Set ${ }^{31}$ allerdings dadurch, dass Letzteres gerade nicht durch eine (syntaktische) Kookkurrenz von FE definiert sein muss, sondern allein die wechselseitige Motivierung desselben KE durch eine Gruppe von FE bezeichnet, wobei die Kookkurrenz im Falle der drei untersuchten Konstruktionen zwar möglich ist, aber kein notwendiges Kriterium für die Konstitution eines Core Set darstellt. Folgerichtig gehen Barsalou \& Hale (1993: 126) davon aus, dass die Kookkurenz von FE nicht vollkommen invariant sein muss, sondern von Instanz zu Instanz, also von Konstrukt zu Konstrukt, unterschiedlich konfiguriert sein kann. Die oben zitierten Belege in (16)-(20) bestätigen dies. Mehr noch: Barsalou \& Hale (1993: 129) spekulieren sogar, dass (rekurrent auftretende?) Kombinationen der Strukturelemente von Frames eigene Sub-Einheiten bilden können. Makro-FE kommen als solche Sub-Einheiten grundsätzlich infrage, mit dem Vorteil, dass sie sich direkt auf die Strukturelemente von Konstruktionen beziehen lassen.

Vergleichbar sind Makro-FE auch zu den Slots im Frame-Modell von Lönneker (2003: 77-79), die Subslots zu Gruppen zusammenfassen. Letztere entsprechen bei Lönneker (2003: 66-74) allerdings nicht den in der Frame-Semantik üblicherweise angenommenen Slots oder Leerstellen und sind demnach auch nicht direkt mit FE zu vergleichen (vgl. Ziem 2008: 328-329, 2014b: 283; Busse 2012: 487-488). Dennoch versteht Busse (2012: 558) Subslots als „Slot-Gruppen“ und rückt sie damit in die Nähe von Core Sets bzw. Makro-FE.

In seinem eigenen Frame-Modell schließlich postuliert Busse (2012: 570) inspiriert durch Barsalou und Lönneker dezidiert „Frame-spezifische[] Gruppen von Frame-Elementen [...], die immer gemeinsam vorkommen müssen, oder sachlich

29 In einer Auseinandersetzung mit klassischen Tiefenkasus wie LocAtion, Source, GoAL und PATH (vgl. Unterabschnitt 2.1.1) begründet Talmy (2007: 71) seine Entscheidung, übergreifend von GRUND in seiner Dichotomie von FIGUR und GRUND zu sprechen - eine Idee, der derjenigen des Makro-FE nahekommt.

30 Barsalou (1992b: 26, Anm. 1) nutzt für die Slots (bzw. FE) eines Frames den Begriff Attribut, den er für synonym erachtet.

31 Interessanterweise fällt bei Barsalou (1992b: 35) explizit der Terminus von „core sets“. Unklar ist, inwiefern die FrameNet-Terminologie sich daran anlehnt, da zumindest bei Ruppenhofer et al. (2016) keine Referenz zu Barsalou zu finden ist. 
zwingende Kovarianten (bzw. Abhängigkeits-Relationen) zwischen einzelnen $\mathrm{Zu}$ schreibungen (Fillern, Werten) festlegen." Sie unterscheiden sich von Core Sets bzw. Makro-FE zwar insofern darin, dass die unter Letzteren subsumierten FE nicht zwingend gemeinsam vorkommen müssen bzw. deren Non-Instanziierung wie oben erwähnt - eher der Regelfall ist. Gleichzeitig decken sie aber gewissermaßen das Phänomen der mehrfachen Instanziierung von KE in durch unterschiedliche FE motivierte KtE ab, das Ausgangspunkt für die Annahme von Makro-FE ist.

Ein Makro-FE, und das ist entscheidend, entspricht für die drei untersuchten Konstruktionen dem KE, dessen KtE einzeln oder mehrfach als FE des Core Set realisiert werden können. Der Name des KE, hier also WEG bzw. $\langle\mathrm{WEG}\rangle$, ist damit gleichzeitig der Name des Makro-FE, dessen einzelne FE die KtE des KE motivieren können. Die Gleichsetzung von KE mit FE, die ich im Eingang zu Kapitel 6 kritisiert habe, ist im Falle des KE Weg bzw. 〈WEG〉 einzig dann möglich, wenn es dem Makro-FE gegenübergestellt wird. Aufgrund der variierenden oder gleichzeitigen Motivierung eines oder mehrerer KtE des KE WEG bzw. 〈WEG〉 durch ein FE des Makro-FE erscheint diese Gleichsetzung nicht auf Ebene der FE, sondern allein auf Ebene des Makro-FE plausibel.

\subsection{Koerzionspotenzial als Teil eines Konstruktionseintrags}

Nachdem ich nun die allgemeine konstruktikographische Beschreibung sowie die Benennung und Definition von KE und KEE diskutiert habe, soll in diesem sowie den folgenden beiden Abschnitten 7.5 und 7.6 um die semantischen Parameter, die als eigene Datenpunkte Eingang in einen Konstruktionseintrag finden müssen, gehen. Das Koerzionspotenzial ist der erste semantische Parameter, der mit einem solchen eigenen Datenpunkt in einen Konstruktionseintrag eingeht (vgl. Unterabschnitt 7.1.3). Bevor dies in der Praxis umgesetzt werden kann, ist es nötig, das Koerzionspotenzial einer Konstruktion zu ermitteln. In Abschnitt 5.5 habe ich bereits auf die Unterschiede zwischen einzelnen Koerzionseffekten und dem generalisierten Koerzionspotenzial einer Konstruktion hingewiesen und dafür argumentiert, dass Koerzion ein graduelles Phänomen ist. Einzelne Koerzionsphänomene in den Konstrukten der betreffenden Konstruktion müssen für die konstruktikographische Verarbeitung dieses Parameters nun zu einem Koerzionspotenzial generalisiert werden. Eine wesentliche Datenquelle dafür sind die einzelnen Konstrukt-Frames, also die semantische Motivierung der KtE und KEE der einzelnen Konstrukte. In den Abschnitten 6.2 und 6.3 habe ich diese Zusammenhänge für beide Arten von Strukturelementen separat für die reflexive Bewegungskonstruktion dargestellt. Für das Koerzionspotenzial ist nun allerdings nicht die Be- 
trachtung eines einzelnen KtE oder KEE von Interesse, sondern die Konstitution des gesamten Konstrukt-Frames.

In diesem Abschnitt stelle ich mittels einer Betrachtung unterschiedlicher Formen der Konstitution eines Konstrukt-Frames sieben Stufen von Koerzionseffekten vor, die sich für die drei untersuchten Konstruktionen ermitteln lassen (Unterabschnitt 7.4.1). Im Anschluss daran präsentiere ich für die reflexive Bewegungskonstruktion (Unterabschnitt 7.4.2), die reflexive Partikelverbkonstruktion (Unterabschnitt 7.4.3) und die reflexive Weg-Konstruktion (Unterabschnitt 7.4.4) jeweils eine quantitative Auswertung dieser sieben Koerzionsstufen, die das Koerzionspotenzial der jeweiligen Konstruktion darstellt. Die so erhobenen Daten können direkt in die drei Konstruktionseinträge im Zusatzmaterial übernommen werden.

\subsubsection{Messung des Koerzionspotenzials: sieben Koerzionsstufen}

Im Zuge der Diskussion des Koerzionspotenzials als semantischer Parameter von Konstruktionen in Abschnitt 5.5 habe ich mit Verweis auf Boas (2011a: 1284-1285) darauf hingewiesen, dass Koerzion alle Strukturelemente eines Konstrukts und nicht nur das Verb einer Argumentstruktur-Konstruktion erfassen kann. Alle KtE sowie das KEE müssen deshalb daraufhin überprüft werden, welcher Anteil ihnen an der Koerzion des gesamten Konstrukts zukommt. Nur dadurch kann in einem ersten Schritt der Koerzionseffekt auf das gesamte Konstrukt bezogen und in einem zweiten Schritt das Koerzionspotenzial der Konstruktion erfasst werden. Letzteres muss also, darauf weist Bybee (2010: 186-187) hin, als eine Eigenschaft der Konstruktion beschrieben werden, die durch die Generalisierung einzelner Koerzionseffekte auf Konstruktebene zustande kommt. Die Analyse der Strukturparallelen zwischen Konstruktionen und Frames und besonders die in den Abschnitten 6.2 und 6.3 für die reflexive Bewegungskonstruktion diskutierten Varianten der semantischen Motivierung von KtE und KEE durch die FE von lexikalischem Frame und Konstruktions-Frame - kurz: die Konstitution von Konstrukt-Frames - ist für diese Aufgabe ein wichtiger Ausgangspunkt. Die Ergebnisse dieser Analysen dienen als Basis für die Bestimmung des Koerzionspotenzials der Konstruktion. Da die semantische Motivierung für jedes KE und das KEE einzeln untersucht wurde, wird der Forderung von Boas (2011a: 1284-1285) damit Rechnung getragen. So ist etwa die doppelte Motivierung eines KtE als Koerzionseffekt - coercion by enrichment im Sinne von Audring \& Booij (2016: 629-631), vgl. Unterabschnitt 6.2.3 - auf der Ebene dieses einzelnen KtE zu verstehen: Das von einem lexikalischen Frame beigetragene FE wird durch ein FE des Konstruktions-Frames koerziert, wobei, um die Metapher von Michaelis (2005: 50) aufzugreifen, beide FE wie Folien ,übereinandergelegt‘ werden und die jeweiligen Eigenschaften der beiden FE glei- 
chermaßen ,durchscheinen'. Die Analyse der semantischen Motivierung von KtE und KEE durch FE ist zunächst eine Analyse von Koerzionseffekten auf der Ebene der Instanziierung einzelner KE bzw. des KEE.

Wie aber lassen sich diese Koerzionseffekte auf Token-Ebene der einzelnen Konstrukt-Frames zu dem Koerzionspotenzial der Konstruktion generalisieren? Ein wichtiger Schritt, um von einzelnen Koerzionseffekten zum Koerzionspotenzial der Konstruktion als Ganzes zu gelangen, ist es, diese Koerzionseffekte nach der Höhe der Koerzion, die in einem Konstrukt auftritt, zu klassifizieren. Ich möchte deshalb zunächst am Beispiel der reflexiven Bewegungskonstruktion für sieben Stufen von Koerzion argumentieren, nach denen sich ihre Konstrukte - genauer: unterschiedlich konstituierte Konstrukt-Frames - einordnen lassen. Relevant ist dabei die Höhe der möglichen Frame-Anpassung eines lexikalischen Frames durch einen Konstruktions-Frame. Um diese Frame-Anpassungen zu untersuchen, müssen die beiden in ihrer semantischen Motivierung besonders varianten Strukturelemente betrachtet werden: die KtE des KE WEG sowie das KEE. Die folgende Aufstellung von sieben Koerzionsstufen richtet sich nach dem graduellen Anteil des Konstruktions-Frames am Konstrukt-Frame und bildet alle Kombinationsmöglichkeiten aus einfacher Motivierung des Strukturelements durch den lexikalischen Frame (Unterabschnitte 6.2.1 und 6.3.1), einfacher Motivierung durch den Konstruktions-Frame (Unterabschnitte 6.2.2 und 6.3.2) und doppelter Motivierung (Unterabschnitte 6.2.3 und 6.3.3) ab. ${ }^{32}$ Je weniger von einem lexikalischen Frame in einem Konstrukt-Frame, übrig bleibt', je stärker der lexikalische Frame also durch den Konstruktions-Frame angepasst wird, desto höher ist der Koerzionseffekt.

- Koerzionsstufe 1: Alle KtE und das KEE sind durch den lexikalischen Frame motiviert.

- Koerzionsstufe 2: Das KtE des KE WeG ist doppelt motiviert und das KEE ist durch den lexikalischen Frame motiviert.

- Koerzionsstufe 3: Das KtE des KE Weg ist einfach durch den KonstruktionsFrame motiviert und das KEE ist durch den lexikalischen Frame motiviert.

- Koerzionsstufe 4: Das KtE des KE Weg und das KEE sind jeweils doppelt motiviert.

- Koerzionsstufe 5: Das KtE des KE Weg ist doppelt motiviert und das KEE ist durch den Konstruktions-Frame motiviert.

32 Genau genommen ergeben sich $3^{2}=9$ Kombinationen, allerdings sind bei einfacher Motivierung eines KtE des KE WEG durch ein FE eines relatierten lexikalischen Frames die beiden Möglichkeiten der einfachen Motivierung des KEE und seiner doppelten Motivierung ausgeschlossen, weshalb noch sieben Kombinationen übrig bleiben. 
- Koerzionsstufe 6: Das KtE des KE WEG ist einfach durch den KonstruktionsFrame motiviert und das KEE ist doppelt motiviert.

- Koerzionsstufe 7: Das KtE des KE WEG und das KEE sind durch den Konstruktions-Frame einfach motiviert.

In Abbildung 7.1 sind diese sieben Koerzionsstufen für die reflexive Bewegungskonstruktion dargestellt, wobei stellvertretend für ein KtE des KE WEG oder für das KEE jeweils angegeben ist, wie sich die semantische Motivierung des jeweiligen Strukturelements zusammensetzt. In der Abbildung ist außerdem zu sehen, wie sich die Staffelung der einzelnen Koerzionsstufen ergibt, dargestellt an dem Anteil des lexikalischen Frames, der sich von zwei FE (Koerzionsstuffe 1) bis hin zu keinem FE (Koerzionsstufe 7) erstreckt. Letzteres entspricht einer maximalen Frame-Anpassung des lexikalischen Frames durch den Konstruktions-Frame (Variante c, vgl. Unterabschnitt 4.4.1). Im Falle einer doppelten Motivierung zählt das FE des lexikalischen Frames ,halb،, wodurch sich die Zwischenstufen 1,5 und 0,5 ergeben.

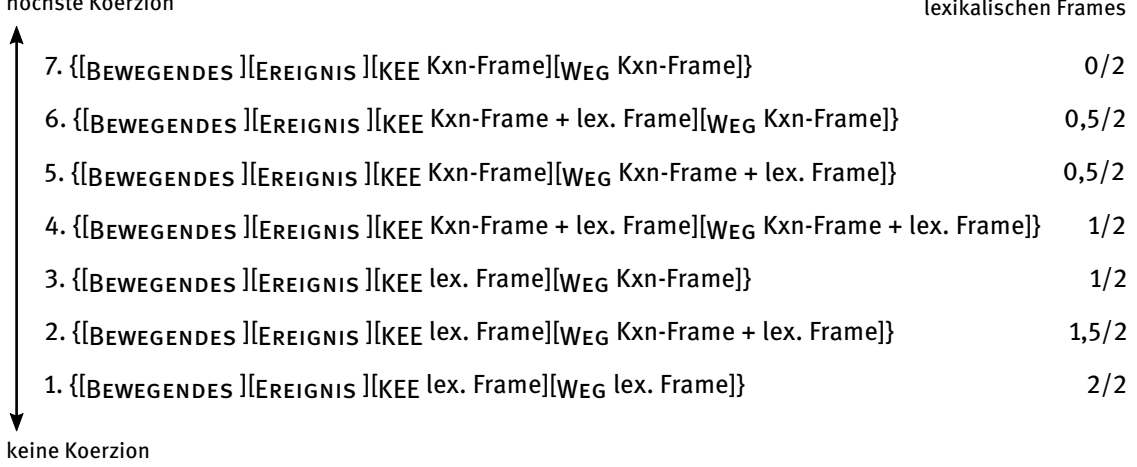

Abb. 7.1: Sieben Koerzionsstufen am Beispiel der reflexiven Bewegungskonstruktion

Diese sieben Koerzionsstufen sind ein Reflex der graduellen Natur von Koerzion (Unterabschnitt 5.5.2) und spiegeln ein Kontinuum wider, das von keiner Koerzion (Koerzionsstufe 1) bis hin zu maximaler Koerzion (Koerzionsstufe 7) reicht. ${ }^{33}$

$33 \mathrm{Zu}$ dieser Auffassung eines Kontinuums zwischen Elaboration und Fusion vgl. auch Hampe \& Schönefeld (2003: 248): „, [T] he distinction between 'fusion' and 'elaboration' is a cline rather than a binary opposition, with verbs (proto-)typically realizing a given construction being positioned at the elaboration-end of the scale.“ Auch für Perek (2015: 40) ist der Unterschied zwischen inher- 
Bevor ich in den Unterabschnitten 7.4.2 bis 7.4.4 das Koerzionspotenzial der drei untersuchten Konstruktionen anhand dieser sieben Koerzionsstufen messen und auf Besonderheiten insbesondere der reflexiven Partikelverbkonstruktion eingehen werde, möchte ich die sieben Koerzionsstufen im Folgenden anhand von Belegen der reflexiven Bewegungskonstruktion illustrieren. Wie bereits in Kapitel 6 nutze ich die Annotation der an der semantischen Motivierung von KtE und KEE beteiligen FE, um die Konstitution der Konstrukt-Frames aufzuzeigen.

In Koerzionsstufe 1 werden alle Strukturelemente des Konstrukts durch FE des lexikalischen Frames motiviert. In diesem Fall findet keine Koerzion statt, da der Konstrukt-Frame keinen Anteil eines separaten Konstruktions-Frames besitzt, der dort gemeinsam mit dem lexikalischen Frame Eingang finden könnte. Es findet also keine Frame-Anpassung statt. Wie in den Unterabschnitten 6.2.1 und 6.3.1 ausgeführt, betrifft die einfache Motivierung aller KtE sowie des KEE ausschließlich relatierte lexikalische Frames (dazu Unterabschnitt 5.4.2). Beispiele für solche Frames habe ich in Kapitel 6 bereits ausführlich diskutiert. An dieser Stelle seien deshalb nur einige Beispiele wiederholt, um die semantische Motivierung der einschlägigen Strukturelemente, der KtE des KE WEG und des KEE, aufzuzeigen.

Wie bereits in Unterabschnitt 6.3.1 betont, steht in den meisten relatierten lexikalischen Frames kein FE für die Motivierung des KEE bereit, da das für das Englische aufgebaute FrameNet die für das Deutsche charakteristischen Reflexivkonstruktionen nicht berücksichtigt. Ich behandele diese Fälle allerdings so, als würde ein entsprechendes FE zur Verfügung stehen, gehe also davon aus, dass auch das KEE semantisch durch den lexikalischen Frame motiviert ist, auch wenn dem KEE kein FE zugeordnet werden kann. Der einfachste Fall betrifft einmal mehr den lexikalischen Frame Motion, für den die Belege in (21) Beispiele sind.

(21) a. BÉDIÉ - er [Motion begab] sich am 3. Januar [Source von seinem Zufluchtsort in Togo aus] nach Paris, wo er eine Wohnung besitzt - wollte damit OUATTARA, der einen burkinabischen Vater hat, von einer Kandidatur ausschließen. (Archiv der Gegenwart, 2001 [2000])

b. Die Indianerin [Motion bewegte] sich in ihren flachen Schuhen beinahe geräuschlos [РАTH durch das Treppenhaus], während die Badelatschen immer lauter gegen Esthers Hacken schlappten. (Düffel, John von: Houwelandt, Köln: DuMont Literatur und Kunst Verlag 2004, S. 128)

\footnotetext{
ent compatibility und semantic enrichment graduell: „[T]he difference between inherent compatibility and semantic enrichment is a matter of gradience rather than a clear-cut distinction, and the position of any composite structure on the continuum between either of these extreme cases depends on how frequently the verb has been witnessed in the relevant construction.“
} 
c. Der Rücken vor ihr, in einem dicken, warmen, grauen Mantel, [Motion bewegte] sich [GoAL zu einem Bahnsteig], Malka folgte ihm. (Pressler, Mirjam: Malka Mai, Weinheim Basel: Beltz \& Gelberg 2001, S. 246)

d. Die augenblickliche Wirtschaftslage [Motion bewege] sich [Direction in Richtung Armut], was das Resultat der ausbeuterischen Privatisierung sei. (Archiv der Gegenwart, 2001 [2000])

Um einen relatierten Frame mit einer Frame-Nähe von +1 heranzuziehen, sei der Frame Self_motion erwähnt. Die Belege in (22) sind Beispiele dafür.

a. So [self_motion hangeln] sich andere Kollegen schon lange [Source von Jahr] zu Jahr - die Unsicherheit werden sie nie los dabei. (Die Zeit, 13.04.2000, Nr. 16)

b. Die Morgensonne [Self_motion stahl] sich schon [РАтн durch die Ritzen der Fensterläden], aber in dem großen Haus war es so still, als schliefen selbst die Bücher in den Regalen. (Funke, Cornelia: Tintenherz, Hamburg: Cecilie Dressler Verlag 2003, S. 94)

c. Ich [self_motion schlich] mich vorsichtig an ihm vorbei [GoAL zu dem Gewürzregal], ließ ihn aber nicht aus den Augen. (Düffel, John von: Vom Wasser, München: dtv 2006, S. 260)

Auch bei Frame-Nähen von +2 und +3 bleibt Koerzionsstufe 1 bestehen, wenn es sich um einen relatierten lexikalischen Frame handelt. Die Belege in (23) sind Beispiele für den lexikalischen Frame Fleeing, der in Vererbungsrelation zum eben erwähnten Frame Self_motion steht, also zu Motion eine Frame-Nähe von +2 besitzt.

(23) a. Nur wer nicht überzeugend politisch handeln kann, [Fleeing flüchtet] sich gern [GoAL in die Welt der PR-Berater undWerbeagenturen]. [sic!] (Tange, Ernst Günter: Zitatenschatz zur Politik, Frankfurt a. M.: Eichborn 2000, S. 84)

b. Diese meine Sätze sind stillschweigend untergegangen mit dem Mauerfall, und ich [Fleeing flüchtete] mich an dieser Stelle [GoAL in den Hauptsatz aller Mitläufer]: (Stadler, Arnold: Sehnsucht, Köln: DuMont Literatur und Kunst Verlag 2002, S. 223)

c. Panik und Niedergeschlagenheit wechselten auch bei ihr ab, sie [Fleeing flüchtete] sich [GoAL zu ihrem chirurgischen Besteck], in ihre Schädel-Hirn-Traumata. (Stadler, Arnold: Sehnsucht, Köln: DuMont Literatur und Kunst Verlag 2002, S. 37) 
Koerzionsstufe 1 besteht letztlich auch bei Konstrukten wie dem in (24), in dem der lexikalische Frame Making_faces in einer Frame-Nähe von +3 zu Motion steht.

(24) [AGENT Ein polnisches Model (Goshia mit Namen) am Tisch gegenüber] versucht sich seit 20 Minuten [〈PATH_OF_GAZE) in unser Gespräch] reinzu[Making_ faces grinsen]. (Die Zeit, 30.03.2000, Nr. 14)

Konstrukt-Frames, die ausschließlich aus lexikalischen Frames bestehen, kommt immer Koerzionsstufe $1 \mathrm{zu}$, in ihnen findet keine Frame-Anpassung und somit keine Koerzion durch den Konstruktions-Frame statt. Alle weiteren Koerzionsstufen betreffen Konstrukt-Frames, die aus FE eines unrelatierten lexikalischen Frames und des Konstruktions-Frames zugleich bestehen. ${ }^{34}$ Die Höhe der Koerzion, die im Einzelfall vorliegt, richtet sich nach der Verteilung dieser beiden Frames (bzw. deren FE) auf die einzelnen Strukturelemente des Konstrukts.

In Koerzionsstufe 2 bleibt die semantische Motivierung des KEE durch den lexikalischen Frame zunächst bestehen. Nun ist das KtE des KE WeG allerdings doppelt motiviert, sodass die Motivierung dieses KtE aus einer Fusion von FE des lexikalischen Frames und des Konstruktions-Frames besteht (vgl. Unterabschnitt 6.2.3). Die Koerzion ist hier noch vergleichsweise gering, da in den beiden einschlägigen Strukturelementen jeweils ein FE des lexikalischen Frames erhalten bleibt, für die Motivierung des KtE des KE WEG wird es allerdings durch die doppelte Motivierung mit dem Konstruktions-Frame um ein FE des KonstruktionsFrames ergänzt. Das FE des lexikalischen Frames, das in die doppelte Motivierung eingeht, zählt somit nur noch ,halb', weswegen der Anteil des lexikalischen Frames, wie in Abbildung 7.1 dargestellt, nur noch 1,5 statt (wie bei Koerzionsstufe 1) 2 beträgt. Wie aus den weiteren Koerzionsstufen noch ersichtlich wird, ist eine doppelte Motivierung eines KtE oder KEE also als weniger koerziv einzustufen als eine einfache Motivierung durch den Konstruktions-Frame, da im ersten Fall noch immer ein Anteil des lexikalischen Frames an der Motivierung zu erkennen ist, während ein solcher Anteil im letzteren Fall nicht mehr gegeben ist.

Ein Beispiel für Koerzionsstufe 2 findet sich in meinen Daten lediglich für einen lexikalischen Frame: Manipulate_into_doing. Beleg (25) enthält das einzige dafür belegte Konstrukt.

34 Vgl. auch Proost (2017: 17), die feststellt, dass Argumentstruktur-Konstruktionen ,show coercion effects when this slot [für das Verb, A.W.] is filled by verbs whose meaning is not straightforwardly related to that of the construction. “ Das Konzept der Frame-Nähe lässt sich also nutzbar machen, um über diese Relatiertheit zu entscheiden: Koerzionseffekte treten (für die reflexive Bewegungskonstruktion) nur bei unrelatierten lexikalischen Frames auf. 
(25) Der Fabrikant breitete sein Schweigen wieder aus, der Offizier [Manipulate_ into_doing mogelte] [Goods sich] [Resulting_Action aus seinem Sitz ${ }^{\text {SourcE }}$. (Düffel, John von: Vom Wasser, München: dtv 2006, S. 56)

In Koerzionsstufe 3 wird die doppelte Motivierung der KtE des KE WEG abgelöst durch eine einfache Motivierung durch den Konstruktions-Frame. Von einem lexikalischen Frame bleibt in der Motivierung der KtE dieses KE also kein Anteil mehr übrig. Gleichzeitig bleibt die einfache Motivierung des KEE durch den lexikalischen Frame bestehen. Der Anteil des lexikalischen Frames beträgt nunmehr also nur noch 1, er ist für die beiden einschlägigen Strukturelemente genauso hoch wie der Anteil des Konstruktions-Frames.

Beispiele für Koerzionsstufe 3 finden sich in meinen Daten, wie solche für Koerzionsstufe 2, nur sehr selten und ebenfalls nur für einen einzigen lexikalischen Frame. Es handelt sich dabei um Cause_to_move_in_place, der selbst über kein FE Source, PATH, GoAL oder Direction verfügt, das nun also vom Konstruktions-Frame beigetragen werden muss, was in einer entsprechenden Frame-Anpassung resultiert. ${ }^{35}$ Die Belege in (26) sind Beispiele dafür.

a. Sie [Cause_to_move_in_place wiegte] [THEme $\operatorname{sich}$ ] [gegen ihn ${ }^{\text {GoAL}}$ ]. (Hettche, Thomas: Der Fall Arbogast, Köln: DuMont Buchverlag, 2001, S. 14)

b. Als sie den Finger auf eine blaue Blüte schob, färbte sich ihr Fingernagel bläulich und grüne Streifen [Cause_to_move_in_place wellten] [THEmE sich] [über ihren Handrücken ${ }^{\mathrm{PATH}}$ ]. (Pressler, Mirjam: Malka Mai, Weinheim Basel: Beltz \& Gelberg 2001, S. 5)

Koerzionsstufe 4 ist hinsichtlich des Anteils des lexikalischen Frames ebenso beschaffen wie Koerzionsstufe 3, mit dem Unterschied, dass nun sowohl das KtE des KE WEG als auch das KEE doppelt motiviert ist. Der Anteil des lexikalischen Frames liegt somit ebenso bei 1. Dadurch, dass ein FE des lexikalischen Frames in der Motivierung der beiden einschlägigen Strukturelemente durch die doppelte Motivierung jeweils nur noch ,halb“ vorhanden ist, erscheint es allerdings gerechtfertigt, diese Art der Motivierung als stärker koerziv als bei der Konstellation von Koerzionsstufe $3 \mathrm{zu}$ betrachten, in der zumindest das KEE noch einfach durch den lexikalischen Frame motiviert war. Grundsätzlich ist die sich in einer doppelten Motivierung eines KtE äußernde Überlappung zwischen lexikalischem Frame und

35 Dass es jedoch für Cause_to_move_in_place auch Belege mit Koerzionsstufe 1 gibt, obwohl es sich um einen unrelatierten lexikalischen Frame handelt, habe ich in Unterabschnitt 6.4.3 (ohne Rückgriff auf den Koerzionsbegriff) gezeigt. Frames können somit nicht pauschal einer bestimmten Koerzionsstufe zugeordnet werden. Ich komme auf diesen Aspekt zum Ende dieses Unterabschnitts zurück. 
Konstruktions-Frame im Rahmen eines Blending-Prozesses (Unterabschnitt 4.1.3) zwar ein ,einfacherer' Fall als die einfache Motivierung eines KtE durch ein FE des Konstruktions-Frame: „[M]eaning-composition is easy when there is appropriate overlap between the conceptual structures to be combined in a blend." (Sweetser 1999: 141). Werden allerdings beide einschlägigen Strukturelemente eines Konstrukts doppelt motiviert und damit von dem Koerzionspotenzial der Konstruktion erfasst, ist diese Art von Koerzion als höher einzustufen als wenn nur, wie in Koerzionsstufe 3, eines der beiden Strukturelemente von dem KonstruktionsFrame erfasst und - auch einfach statt doppelt - motiviert wird..$^{36}$

Eine weitere Evidenz dafür, dass die doppelte Motivierung beider Strukturelemente als koerziver einzustufen ist, besteht in der durchaus großen Bandbreite lexikalischer Frames, die für Koerzionsstufe 4 für die reflexive Bewegungskonstruktion belegt sind. Der mit Abstand frequenteste lexikalische Frame für diese Koerzionsstufe ist Cause_to_amalgamate. Einige Belege für ihn sind in (27) zusammengestellt.

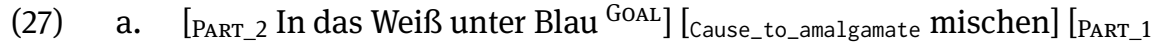
sich ${ }^{\text {THEME }}$ d die Wolkenschatten und die dunklen Birkenstoppeln in den Senken. (Die Zeit, 05.01.2000, Nr. 2)

b. Die beiden Frauen [cause_to_amalgamate mischen] [PART_1 sich THEME] [PART_2 unter die Zuschauertraube ${ }^{\text {GoAL] }}$. (Krausser, Helmut: Eros, Köln: DuMont 2006, S. 131)

c. Unverständliches Genuschel [Cause_to_amalgamate mischte] [PART_1 sich THEME] [PART_2 Zwischen einzelne Schluchzer ${ }^{\text {GOAL }}$ ], es war unmöglich zu sagen, ob das Dienstmädchen aus Rührung, Empörung oder Eifersucht weinte. (Düffel, John von: Vom Wasser, München: dtv 2006, S. 206)

d. Lichtgarben und Wolkenschatten [Cause_to_amalgamate mengen] [PART_1 sich $\left.^{\text {THEME }}\right]_{\text {PART_2 }}$ in den Strom, der in seiner Meeresgrüne schäumend aufblitzt ${ }^{\text {GoAL }}$. (Düffel, John von: Vom Wasser, München: dtv 2006, S. 69)

36 Inwiefern eine doppelte Motivierung sichtbare Gegenstück-Relation zwischen den FE von lexikalischem Frame und Konstruktions-Frame auf eine konzeptuelle Nähe dieser hinweist, worauf ich zu Beginn von Abschnitt 5.4 in Anlehnung an Haiman (1983: 783) hingewiesen habe (vgl. auch Unterabschnitt 6.2.3), sei an dieser Stelle offen gelassen und bietet Ansätze für zukünftige Forschungen zum Thema Frame-Nähe, die sich nicht nur auf Frame-zu-Frame-Relationen stützen, sondern frequente doppelte Motivierungen zwischen zwei Frames (Konstruktions-Frame und lexikalischem Frame) in den Blick nehmen könnten. 
e. Ein Lied singt sie zusammen mit Armand, [cause_to_amalgamate fädelt] [PART_1 sich ${ }^{\text {THEME}}$ [PART_2 $_{\text {in seine Töne }}{ }^{\text {GoAL }}$ ], und er spielt sie an, fällt vor ihr auf die Knie, kann ihr so unter den Rock gucken. (Dölling, Beate: Hör auf zu trommeln, Herz, Weinheim: Beltz \& Gelberg 2003, S. 205)

f. Im Regen [Cause_to_amalgamate fädelte] [PART_1 sich $^{\text {THEME}}$ ] das Taxi mit wütenden Geräuschen [PART_2 in die Wagenschlange auf der Stadtautobahn ein ${ }^{\mathrm{GOAL}}$ ]. (Riedel, Susanne: Eine Frau aus Amerika, Berlin: Berlin Verlag 2003, S. 15)

Weitere Beispiele für Koerzionsstufe 4 finden sich mit den lexikalischen Frames Emptying (28) und Filling (29).

(28) a. „Wie einer der jetzt nochmals vernommenen Beamten ja erst ges-

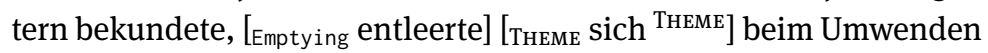
und beim Abtransport der Leiche Blutflüssigkeit [Source aus der Nase SouRCE]. (Hettche, Thomas: Der Fall Arbogast, Köln: DuMont Buchverlag, 2001, S. 312)

b. „Welche Landschaft?“ frage ich, [Emptying schäle] [Theme mich ${ }^{\text {THEmE] }}$ vorsichtig [SOURCE aus der Decke SourcE] und schaue mich um. (Riedel, Susanne: Eine Frau aus Amerika, Berlin: Berlin Verlag 2003, S. 74)

(29) Die leuchtendgelbe Schale einer Zitrone [Filling wickelt] [Tнеме sich $^{\text {THеме] }}$

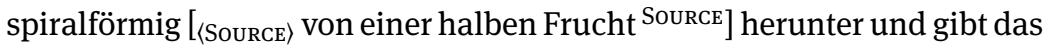
hellere Fleisch und die weißliche Innenhaut frei. (Noll, Ingrid: Ladylike, Zürich: Diogenes 2006, S. 289)

Die folgenden beiden Belege lassen sich für die lexikalischen Frames Attaching (30) und Smuggling (31) finden.

(30) Spinnenweb [Attaching klebte] [Iтte sich $^{\text {Theme] }}$ [Goal in sein Gesicht ${ }^{\text {Goal }}$ ]. (Koneffke, Jan: Paul Schatz im Uhrenkasten, Köln: DuMont Buchverlag 2000, S. 216)

(31) Und wenn die Jungs nach getaner Tat in den Armen der Liebsten schlummern, [smuggling schmuggelt] [Goods sich $^{\text {THEME}}$ ] Rudi mittels Dollars und Autogrammfußbällen am Endlosstau von Brest vorbei [GoAL nach Polen ${ }^{\text {GoAL }}$ ] rein. (Die Zeit, 24.02.2000, Nr. 9)

Als letztes Beispiel für Koerzionsstufe 4 soll der lexikalische Frame Grinding dienen, für den das in (32) enthaltene Konstrukt belegt ist. 
(32) Ein Motor dröhnt von der Straße herüber, und diese dröhnende Straße [Grinding fräst] [PATIENT sich $^{\text {THEME}}{ }^{\text {[GoAL }}$ in mein Gedächtnis ${ }^{\text {GOAL }}$ ] ein. (Riedel, Susanne: Eine Frau aus Amerika, Berlin: Berlin Verlag 2003, S. 95)

In Koerzionsstufe 5 sinkt der Anteil des lexikalischen Frames noch einmal. Im Unterschied zu Koerzionsstufe 4 ist das KEE nun einfach durch den KonstruktionsFrame motiviert, während das KtE des KE WEG nach wie vor doppelt motiviert ist. Der Anteil des lexikalischen Frames beträgt nunmehr noch 0,5.

Die lexikalischen Frames, die für diese Koerzionsstufe belegt sind, sind noch zahlreicher als für Koerzionsstufe 4, deswegen seien im Folgen nur einige Belege zur Illustration zitiert. Unter den relevanten lexikalischen Frames findet sich etwa Seeking, für den die Konstrukte in (33) Beispiele sind.

(33) a. Die jetzt 30-köpfige Truppe der Poststaatsfarmer [seeking tappt] [sich THEME] nach Hörensagen [Sought_ENTITY an EU-Vorschriften ${ }^{\text {GOAL}}$ ] heran, entsorgt die Gülle bodenschonend, gibt den Schweinen mehr Kräuter als Hormone. (Die Zeit, 16.03.2000, Nr. 12)

b. In Österreich [seeking tastet] [sich ${ }^{\text {THEME}] ~ d i e ~ G y n a ̈ k o l o g e n s c h a f t ~, v o r-~}$ sichtig und abwartend" [Sought_entity an das Präparat heran GoAL]. (Die Zeit, 27.04.2000, Nr. 18)

c. Langsam wagen sie sich heran, [seeking tasten] [sich ${ }^{\text {THEME}}$ ] [Sought_entiTY auf meinen vom Schmerz durchwaberten Rücken ${ }^{\text {GoAL }}$; die ungeheuerliche Berührung, als testeten sie, ob oder auf welche köstliche Weise ihre Beine wohl einsinken in diesem seltenen eßbaren Grund. (Braun, Marcus: Hochzeitsvorbereitungen, Berlin: Berlin Verlag 2003, S. 113)

d. Die Frau nimmt nicht den Fahrstuhl, sondern [seeking tastet] [sich THEME] über die Treppen hinauf [Sought_ENTITY in den sechsten Stock GoAL], vorbei an den schlafenden Bettlern, die der heilige Martin vergaß. (Venske, Regula: Marthes Vision, Frankfurt am Main: Eichborn Verlag 2006, S. 184)

e. Er ruckelte und scharrte, bis er die Schaufel voll glaubte, [seeking tastete] [sich ${ }^{\text {THEME] }}$ rückwärts gehend [Sоught_ENTITY $z u$ den Fahrrädern

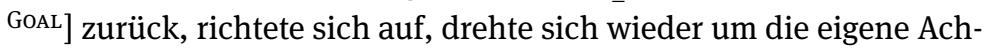
se und ging, aufrecht nun und die volle Schaufel waagrecht an einer Seite tragend, an den Kisten und Koffern vorbei, die zwei Stufen zur Heizung hinunter. (Widmer, Urs: Das Buch des Vaters, Zürich: Diogenes 2004, S. 167)

Auch der bereits häufiger illustrierte lexikalische Frame Work gehört mit zahlreichen Konstrukten zu Koerzionsstufe 5, wie die Belege in (34) zeigen. 
(34) a. Die Ermittler [work arbeiteten] [sich ${ }^{\text {THEME}}$ ] [SALIEnt_entity an den großen Zampano ${ }^{\mathrm{GoAL}}$ ] heran. (Die Zeit, 27.04.2000, Nr. 18)

b. Sie hörte das knarrende Geräusch, als die Scherenblätter aufgingen, spürte das Metall an ihrem Hals und dann [work arbeitete] [sich THEME] die Schere säbelnd und schabend [SALIENT_ENTITy durch ihren Zopf ${ }^{\text {PatH }}{ }^{2}$. (Pressler, Mirjam: Malka Mai, Weinheim Basel: Beltz \& Gelberg 2001, S. 269)

c. Putin [work arbeitete] [sich ${ }^{\text {THEME}}$ ] mit Fleiß und Stetigkeit [SAlient_Entity nach oben ${ }^{\text {GoAL }}$, nicht mit Brillanz. (Die Zeit, 23.03.2000, Nr. 13)

d. Man begann bei den Gesetzen der Körperwelt, [work arbeitete] [sich Theme] empor [Salient_Entity zur Psychologie GoAL], zu den Gesetzen des Empfindens und Denkens, Ausflüge in die Schöne Literatur waren vorgesehen, sie ermöglichten den Übergang zum Feinseelischen und zu den letzten Fragen, Gott und Unsterblichkeit. (Safranski, Rüdiger: Friedrich Schiller, München Wien: Carl Hanser 2004, S. 46)

Gleiches gilt für Ingestion und die Belege in (35).

a. Also [Ingestion aßen] [sich THEme] Kohl und Rühe tapfer [Ingestibles durch große Stücke Marzipantorte $\left.{ }^{\mathrm{PATH}}\right]$, unter der Aufsicht von mindestens 15 Kamerateams und unter den kritischen Blicken eines ganzen Schwarms von Reportern, die lauerten und hofften - worauf eigentlich? (Die Zeit, 27.01.2000, Nr. 5)

b. Abends las ich, die Bücherei war ziemlich umfangreich, und ich

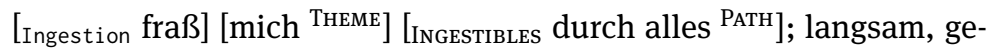
nügsam, manchmal mit wirklicher Anteilnahme. (Schmitter, Elke: Frau Sartoris, Berlin: BvT 2000[2002], S. 35)

c. Er spürte wieder jenen Würgegriff um den Schädel, den tödlichen Druck vom Nasenbein her; wie eine Säure [Ingestion fraß] [sich THEmE] die Angst noch einmal [Ingestibles in seine Bauchhöhle ${ }^{\text {GoAL] }}$ und die Verzweiflung, mit der sich damals sein Leben, sein einziges, eigenes Leben zum ersten Mal als verlorenes zu erkennen gegeben hatte. (Hahn, Ulla: Unscharfe Bilder, München: Deutsche Verlags-Anstalt 2003, S. 170)

Zwei weitere exemplarisch zu erwähnende Frames sind Scouring (36) und Desiring (37).

a. Von Jakarta bis Seoul - überall [scouring wühlen] [sich THEmE] Wirtschaftsprüfer, Weltbanker und Experten des Internationalen Wäh- 
rungsfonds [GRound durch Bankbilanzen ${ }^{\text {PATH }}$. (Die Zeit, 17.02.2000, Nr. 8)

b. Er klammerte sich so fest daran, dass seine kurzen Fingernägel [sich THEME] [GRound in ihre Haut GoAL] [scouring gruben]. (Funke, Cornelia: Tintenherz, Hamburg: Cecilie Dressler Verlag 2003, S. 320)

a. Mancher Programmdirektor [Desiring wünscht] [sich ${ }^{\text {THEME}}$ ] hier inzwischen [Event auf eine wirklich einsame Insel ${ }^{\mathrm{GOAL}}$ ]. (Die Zeit, 30.03. 2000, Nr. 14)

b. Er [Desiring wünscht] [sich ${ }^{\text {THEME}}$ ], von der Vaterwelt enttäuscht, [EvenT in den Mutterleib ${ }^{\text {GoAL }}$ zurück, deshalb fügt es sich gut, daß in diesem Augenblick seine Spießgesellen einen Räuberhauptmann suchen. (Safranski, Rüdiger: Friedrich Schiller, München Wien: Carl Hanser 2004, S. 113)

Die vorletzte Koerzionsstufe 6 steht hinsichtlich des Anteil des lexikalischen Frames erneut auf demselben Rang wie die Koerzionsstufe zuvor. Die semantische Motivierung der beiden einschlägigen Strukturelemente ist nun allerdings umgekehrt: War bei Koerzionsstufe 5 noch das KEE einfach durch den KonstruktionsFrame und das KtE des KE WEG doppelt motiviert, ist nun das KEE doppelt und das KtE des KE WEG einfach durch den Konstruktions-Frame motiviert. Die Reihenfolge der beiden Varianten, die Koerzionsstufe 5 und 6 zukommen, ist also noch weniger relevant als dies bei den Koerzionsstufen 3 und 4 der Fall ist. Wie bei Koerzionsstufe 3 auch wird der Konstrukt-Frame nun in jedem Fall durch ein FE SourCE, PATH, GoAl oder DiRection ergänzt, das im lexikalischen Frame nicht angelegt ist oder nicht mit einem von dessen FE fusionieren kann.

Zwei für Koerzionsstufe 6 belegte lexikalische Frames sind in den Belegen in (38) mit Rescuing und in (39) mit dem frequenten Frame Cause_to_experience zu sehen.

(38) a. Zehntausende von Überlebenden [Rescuing retteten] [PATIENT sich $^{\text {THEME] }}$ [auf Hügel, Bäume und Hausdächer GoAL], wo sie ohne Trinkwasser und Nahrungsmittel teilweise tagelang ausharrten, bis sie von Hubschraubern gerettet und in Sammellager geflogen wurden. (Archiv der Gegenwart, 2001 [2000])

b. Mommsen [Rescuing rettete] [Patient sich $^{\text {ThEME] }}$ [aus der Welt der Tatsachen ${ }^{\text {SOURCE}}$ ] in die der Gefühle: (de Bruyn, Günter: Preußens Luise, Siedler 2001, S. 87)

c. Ich weiß, daß sie mir alles aus dem Gesicht ablesen kann, will es nicht dazu kommen lassen, werde immer linkischer und [Rescuing ret- 
te] [PATIENT mich ${ }^{\text {THEME }}$ [in die Einsilbigkeit ${ }^{\text {Goal }}$. (Schrott, Raoul: Tristan da Cunha oder die Hälfte der Erde; Hanser Verlag 2003, S. 513)

(39) a. Sie [cause_to_experience quälten] [EXPERIENCER sich ${ }^{\text {THEME}}$ ] vielleicht auch [aus Tischen ${ }^{\text {SourcE}}$, wenn man voller Zweifel dagegen stieß? (Kuckart, Judith: Lenas Liebe, Köln: DuMont Literatur und Kunst Verlag 2002, S. 123)

b. Phillip redet darüber, wie blöd ein Buch war, das er gelesen hat: ,... und ich hab [EXPERIENCER mich THEME] [durch dreihundertzweiundfünfzig Seiten ${ }^{\mathrm{PATH}}$ ] [cause_to_experience gequält] und danach konnte ich einfach nur sagen: ,Ach, und das war 's? (Bach, Tamara: Marsmädchen, Hamburg: Verlag Friedrich Oetinger 2003, S. 40)

c. Vom sonnigen unteren Centovalli aus steigt man über fette Feuersalamander durch Esskastanienwälder bergan, schwitzt, dampft, springt nackt in einen Bergbach, [Cause_to_experience quält] [EXPERIENCER sich THEME] [über verrutschte Pfade ${ }^{\text {PATH}}$ ] an Ziegenvolk vorbei ins Baumlose, zieht einen Pullover an, wirft den ersten Schneeball, und bei 1000 Metern über null versinkt man bis zur Hüfte im Schnee. (Die Zeit, 30.03.2000, Nr. 14)

d. Lukian [cause_to_experience quält] [EXPERIENCER sich $^{\text {THEME] }}$ [zu einem Lächeln $\left.{ }^{\mathrm{GOAL}}\right]$. (Krausser, Helmut: Eros, Köln: DuMont 2006, S. 146)

In der höchsten Koerzionsstufe 7 enthält die semantische Motivierung der beiden Strukturelemente, des KtE des KE WEG und des KEE, keinen Anteil des lexikalischen Frames mehr. Beide Strukturelemente werden durch den KonstruktionsFrame motiviert, es liegt eine maximale Frame-Anpassung des lexikalischen Frames vor (Variante $c$ in Unterabschnitt 4.4.1). Für die reflexive Bewegungskonstruktion korreliert diese Koerzionsstufe, darauf habe ich in Unterabschnitt 5.2.3 bereits - ohne Rückgriff auf den Koerzionsbegriff - hingewiesen, mit der, incidental'Lesart der Konstruktion. Konstrukte, deren Konstrukt-Frame entsprechend Koerzionsstufe 7 konstituiert ist, kommen also besonders für diese Lesart infrage. Die in Unterabschnitt 7.2.2 angesprochene Korrelation zwischen den Lesarten und deren Token-Frequenzen lässt sich also durch die Verteilung der Konstrukte auf die sieben Koerzionsstufen feiner abbilden.

Für diese Koerzionsstufe ist erneut eine große Bandbreite lexikalischer Frames belegt, sodass die im Folgenden zitierten Belege lediglich der Illustration dienen. Ein frequent belegter Frame ist Daring, für den die Belege in (40) Beispiele sind.

(40) a. Aber früher, als wir noch klein waren, [Daring traute] [sich ${ }^{\text {THEME}] ~ n i e-~}$ mand [an uns ${ }^{\text {GoAL }}$ ] heran, auch nicht in Mückes Gegend, denn wir 
standen unter dem Schutz seines Bruders, und dessen Rache würde fürchterlich sein. (Goosen, Frank: Liegen lernen, Frankfurt am Main: Eichborn AG 2000, S. 88)

b. Nur [Daring traue] [sich ${ }^{\text {THEmE}] ~ h o ̈ h e r e n ~ O r t s ~ n i e m a n d ~[a n ~ d a s ~ h e i k l e ~}$ Thema ${ }^{\text {GoAL }}$ ] heran. (Die Zeit, 24.02.2000, Nr. 9)

c. Er meidet diese Gegend, [Daring wagt] [sich ${ }^{\text {THEME}}$ ] nicht [auf den Hügel GoAL] herauf, fürchtet noch den entferntesten Blickkontakt mit einem Mitglied seiner Familie. (Beyer, Marcel: Spione, Köln: DuMont 2000, S. 126)

d. Wie die Franzosen platzierten sie ihre Anlagen an den attraktivsten Stränden rund ums Mittelmeer und [Daring wagten] [sich ${ }^{\text {THEME}] ~ b a l d ~}$ auch [auf andere sonnenverwöhnte Erdteile ${ }^{\mathrm{GOAL}}$ ] vor, in denen immer sommerliche Temperaturen herrschten. (Die Zeit, 09.03.2000, Nr. 11)

e. Er [Daring traute] [sich ${ }^{\text {THEME}}$ ] nicht mehr [aus dem Haus SOURCE], hörte immer wieder Stimmen, fühlte sich bedroht und sah im Spiegel jemanden, den er nicht kannte. (Die Zeit, 09.03.2000, Nr. 11)

f. Hanna wusste nicht, was sie denken sollte, sie [Daring wagte] [sich ${ }^{\text {THEмE] }}$ nicht zurück [in die Halle ${ }^{\text {GoAL}}$ ], weil sie Angst vor den fragenden Blicken Minnas hatte, vor den abwiegelnden Bemerkungen ihrer Weggenossen. (Pressler, Mirjam: Malka Mai, Weinheim Basel: Beltz \& Gelberg 2001, S. 208)

g. Er machte sich Vorwürfe und [Daring getraute] [sich ${ }^{\text {THEME}]}$ aus schlechtem Gewissen nicht einmal mehr [zu seinen Kindern GoAL]. (Wondratschek, Wolf: Mozarts Friseur, München, Wien: Carl Hanser Verlag 2002, S. 79)

Nahezu ebenso frequent und bereits häufiger zitiert ist der lexikalische Frame Hostile_encounter. Wie Koerzionsstufe 7 sich in Konstrukt-Frames mit ihm auswirkt, ist in den Belegen in (41) zu sehen.

(41) a. Der Wagen legte kaum zweihundert Meter in der Minute zurück,

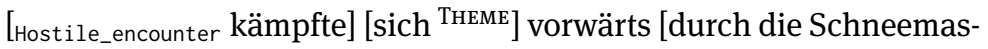
sen ${ }^{\text {PATH }}$, bog von der Landstraße ab in eine von wenigen Laternen beleuchtete Allee. (Krausser, Helmut: Eros, Köln: DuMont 2006, S. 8)

b. Maria [Hostile_encounter ringt] [sich ${ }^{\text {THEME] }}$ in Wirklichkeit [zu einer inneren Freiheit durch, die es ihr erlaubt, Verantwortung für ihr Leben zu übernehmen, das ungerechte Urteil und den Tod als Sühne hinzunehmen und in einen Akt der Freiheit zu verwandeln ${ }^{\text {GoAL }}$. (Safranski, Rüdiger: Friedrich Schiller, München Wien: Carl Hanser 2004, S. 478) 
Als zwei weitere lexikalische Frames seien, unter zahlreichen anderen, Make_noi se (42) und Dead_or_alive (43) erwähnt.

a. Sie [Make_noise klicken] [sich ${ }^{\text {THEME }}$ [ [durch Portale ${ }^{\text {PATH }}$, orientieren sich an Sitemaps, immer auf der Suche nach der besten Adresse. (Die Zeit, 03.02.2000, Nr. 6)

b. „ „... für die vielen Toten und Verwundeten hergeschickt worden waren, nachgerückt wie Kegel im Spiel“, machte der Vater seinen Fehler wieder gut, ,junge Kerle, gerade Abitur gemacht, [Make_noise heulten] [sich ${ }^{\text {THEME }] ~ a u f ~ i h r e n ~ S t r o h s c h u ̈ t t e n ~ i n ~ d e n ~ E r d b u n k e r n, ~ w o ~ w i r ~ u n s ~}$ zusammendrängten, [in den Schlaf GoAL]. (Hahn, Ulla: Unscharfe Bilder, München: Deutsche Verlags-Anstalt 2003, S. 103)

(43) Man [Dead_or_alive lebte] [sich ${ }^{\text {THEME}] ~[a u s ~ d e r ~ h e r a u s g e r i s s e n e n ~ Z e i t, ~ i n ~ d e r ~}$ man saß Source], zurück in die Erinnerung ans Zuhause von damals und voraus in die Hoffnung, bald heimzukehren. (Müller, Herta: Der König verneigt sich und tötet, München: Carl Hanser Verlag 2003, S. 42)

Nach dieser Darstellung der sieben Koerzionsstufen möchte ich noch auf eine Notwendigkeit der Differenzierung hinweisen. Aus der bisherigen Diskussion könnte der Eindruck entstehen, dass bestimmte lexikalische Frames bestimmten Koerzionsstufen fest zuzuordnen sind. Wenngleich dies in vielen Fällen in meinen Daten tatsächlich möglich ist, lassen sich einige Frames finden, die - je nach Konstitution des Konstrukt-Frames - in mehreren Koerzionsstufen auftreten. Ein Beispiel ist der Frame Work, den ich bereits als Beispiel für Koerzionsstufe 5 erwähnt habe. Betrachtet man nun allerdings die Belege in (44), in denen die KtE des KE WEG nicht doppelt, sondern einfach durch den Konstruktions-Frame motiviert sind, sind diese der Koerzionsstufe 7 zuzuordnen. Entscheidend ist also die konkrete semantische Motivierung der einzelnen Strukturelemente eines Konstrukts. Lexikalische Frames lassen sich nicht pauschal bestimmten Koerzionsstufen zuordnen.

(44) a. Irgendein ferner Schmerz [work arbeitet] [sich THEME] [auf mich GoAL] zu, ich sehe ihn kommen. (Genazino, Wilhelm: Die Liebesblödigkeit, München, Wien: Carl Hanser Verlag 2005, S. 108)

b. „Manchmal [work arbeiten] [sich THEME] [aus der teigigen, formlosen Volksmasse SOURCE] irgendwelche besonderen, starken, sehr arbeitsfähigen Menschen nach oben. (Schlögel, Karl: Petersburg, München Wien: Carl Hanser Verlag 2002, S. 292)

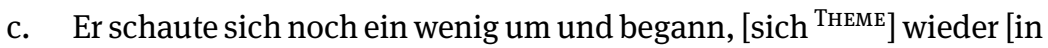
Richtung Ausgang Direction] vorzu[work arbeiten]. (Suter, Martin: Lila, Lila, Zürich: Diogenes 2004, S. 344) 
d. Sein Herz [work arbeitete] [sich ${ }^{\text {THEmE }}$ ] voran, immer höher, immer dichter [unter die Haut GoAL]. (Schulze, Ingo: Neue Leben, Berlin: Berlin Verlag 2005, S. 751)

Ein anderes Beispiel für diesen Fall ist Seeking, den ich ebenfalls bereits für Koerzionsstufe 5 erwähnt habe. Da in den Belegen in (45) aber ebenfalls keine doppelte Motivierung der KtE des KE WEG vorliegt, fallen diese Konstrukte in Koerzionsstufe 7 .

(45) a. Wir [seeking tasteten] [uns ${ }^{\text {THEME}] ~ l a n g s a m ~[d u r c h ~ d a s ~ D u n k e l, ~ i n ~ d e m ~}$ es kein Ziel, keine Richtung mehr gab, nur den Gestank von totem Wasser und das schwache Tröpfeln der Rinnsale, unterbrochen vom platschenden Schritt unserer unsichtbaren Füße und dem Stöhnen unseres beklommenen Atems ${ }^{\text {РАTH}}$ ]. (Düffel, John von: Vom Wasser, München: dtv 2006, S. 39)

b. Die Frau nimmt nicht den Fahrstuhl, sondern [seeking tastet] [sich THEME] [über die Treppen ${ }^{\mathrm{PATH}}$ ] hinauf in den sechsten Stock, vorbei an den schlafenden Bettlern, die der heilige Martin vergaß. (Venske, Regula: Marthes Vision, Frankfurt am Main: Eichborn Verlag 2006, S. 184)

$\mathrm{Zu}$ diesem Zweck sei insbesondere Beleg (45-b) mit seiner bereits für ein anderes KtE der KE WEG zitierten Annotation verglichen, die ich in (46) wiederhole. Da das KE WEG in diesem Konstrukt mehrfach instanziiert ist (Unterabschnitt 6.4.1), lassen sich die Unterschiede daran gut aufzeigen.

(46) Die Frau nimmt nicht den Fahrstuhl, sondern [seeking tastet] [sich THEME] über die Treppen hinauf [Sought_EnTity in den sechsten Stock GoAL], vorbei an den schlafenden Bettlern, die der heilige Martin vergaß. (Venske, Regula: Marthes Vision, Frankfurt am Main: Eichborn Verlag 2006, S. 184)

Während das KtE über die Treppen nicht als mit dem FE Seeking.SouGHT_ENTITY doppelt motiviert angesehen werden kann, gilt dies für das KtE in den sechsten Stock schon eher. Im ersteren Fall gehört das Konstrukt zu Koerzionsstufe 7, im letzteren zu Koerzionsstufe 5. Da das Konstrukt aufgrund der mehrfachen Instanziierung des KE WEG durch die nach Präpositionen getrennten Suchanfragen an das DWDS-Kernkorpus 21 (Unterabschnitt 3.4.1) ohnehin mehrfach in den Daten erscheint, ist es also unproblematisch, es, je nachdem welches KtE gerade relevant ist, mehreren Koerzionsstufen zugleich zuzuordnen. 
Ein Frame, der ebenfalls in mehreren Koerzionsstufen auftritt, ist Ingestion, dessen Beleg (47) ebenfalls Koerzionsstufe 7 und nicht, wie andere Belege für ihn, Koerzionsstufe 5 zuzuordnen ist.

(47) Es war allen Anwesenden nachzufühlen; von unschuldigem Frohsinn war nichts zu spüren, die Abende zogen sich dahin, der Steuereintreiber und der Chirurg entschuldigten sich schon früh, um nicht wieder in den Genuß von Gratiskonsultationen zu kommen, der Priester gab seine zwei Witze zum besten, und der Rest lästerte über das Essen und [Ingestion soff] [sich THEмE] [unter den Tisch GoAL], weil selbst die Konjunktur als Thema längst ausgeschöpft war. (Schrott, Raoul: Tristan da Cunha oder die Hälfte der Erde; Hanser Verlag 2003, S. 196)

Da nun die grundlegenden Prinzipien zur Klassifikation der Koerzionseffekte feststehen, lassen sich anhand der Zuordnung entsprechender Konstrukte zu den einzelnen Koerzionsstufen die Koerzionspotenziale für die drei untersuchten Konstruktionen bestimmen. Dadurch wird ein Vergleich der drei Konstruktionen möglich.

\subsubsection{Koerzionspotenzial der reflexiven Bewegungskonstruktion}

Die Feststellung der sieben Koerzionsstufen, wie ich sie in Unterabschnitt 7.4.1 für die reflexive Bewegungskonstruktion getroffen habe, kann direkt zu einer quantitativen Auswertung der Verteilung dieser Koerzionsstufen über die Konstrukte der Konstruktion hinweg übergehen. Für die reflexive Bewegungskonstruktion ist in Tabelle 7.15 dargestellt, wie viele Konstrukte auf jede Koerzionsstufe entfallen und wie hoch deren Anteil im Vergleich zur Gesamtanzahl der Konstrukte der Konstruktion ist.

Auf den ersten Blick ist deutlich zu erkennen, dass Koerzionsstufe 1 mit Abstand am frequentesten ist, die meisten Konstrukte der Konstruktion also auf relatierte lexikalische Frames entfallen. Diese Ergebnisse sind bereits aufgrund der Analysen in den Abschnitten 6.2 und 6.3 erwartbar, da bei der dortigen Auswertung der lexikalischen Frames, die eine einfache Motivierung aller KtE und des KEE bewirken, die hohe Frequenz von Konstrukten mit relatierten lexikalischen Frames bereits implizit deutlich wurde.

Insbesondere die Verteilung der Koerzionsstufen 2 bis 7, die das eigentliche Koerzionspotenzial der Konstruktion ausmachen - da in Koerzionsstufe 1 ja, wie in Unterabschnitt 7.4.1 gesehen, keine Koerzion stattfindet -, ist, wie sie in Tabelle 7.15 dargestellt ist, für eine einzelne Konstruktion allerdings wenig aussagekräf- 
Tab. 7.15: Koerzionspotenzial der reflexiven Bewegungskonstruktion

\begin{tabular}{lrr}
\hline Koerzionsstufe & Konstrukte & Anteil \\
\hline 1 & 830 & $82,10 \%$ \\
2 & 1 & $0,10 \%$ \\
3 & 2 & $0,20 \%$ \\
4 & 40 & $3,96 \%$ \\
5 & 50 & $4,95 \%$ \\
6 & 33 & $3,26 \%$ \\
7 & 55 & $5,44 \%$ \\
\hline Gesamt & 1.011 & $100,00 \%$ \\
\hline
\end{tabular}

tig. Ob das Koerzionspotenzial der reflexiven Beweungskonstruktion nun als hoch oder niedrig einzustufen ist, lässt sich freilich nur durch einen Vergleich mit anderen Konstruktionen beantworten. Hierfür kommen natürlich zuvorderst die zur reflexiven Bewegungskonstruktion verwandten Konstruktionen infrage, also die reflexive Partikelverbkonstruktion und die reflexive Weg-Konstruktion. Auf die Verteilung der sieben Koerzionsstufen auf ihre Konstrukte möchte ich deshalb in den folgenden beiden Unterabschnitten 7.4.3 und 7.4.4 eingehen und damit die Grundlage für einen Vergleich zur reflexiven Bewegungskonstruktion schaffen, um die Höhe des jeweiligen Koerzionspotenzials der drei Konstruktionen abschließend beurteilen zu können.

\subsubsection{Koerzionspotenzial der reflexiven Partikelverbkonstruktion}

Die reflexive Partikelverbkonstruktion ist in ähnlichem Maße wie die reflexive Bewegungskonstruktion von Koerzionseffekten betroffen. Der abnehmende Anteil eines lexikalischen Frames an der Konstitution von Konstrukt-Frames, wie er sich in den für die reflexive Bewegungskonstruktion formulierten sieben Koerzionsstufen (Unterabschnitt 7.4.1, Abbildung 7.1) äußert, lässt sich nahezu analog für die reflexive Partikelverbkonstruktion beobachten. Schon Knobloch (2009: 548) hält allgemein fest: „Je stärker die Beteiligung der lexikalischen Verbbedeutung an den Partikelverbkonstruktionen zurücktritt, desto deutlicher tritt die Beteiligung von Partikel plus Konstruktion in den Vordergrund.“ Das Koerzionspotenzial der reflexiven Partikelverbkonstruktion kann analog zur reflexiven Bewegungskonstruktion mit sieben Koerzionsstufen erfasst werden, allerdings ist für sie das Charakteristikum der Einstufung des KE 〈WEG〉 als Nicht-Kern-KE zu berücksichtigen, weshalb die Zuordnung der Konstrukte auf die sieben Koerzionsstufen für die reflexive Partikelverbkonstruktion angepasst werden muss. 
Bevor die Verteilung der Konstrukte der reflexiven Partikelverbkonstruktion auf die sieben Koerzionsstufen ermittelt und ein Vergleich zu den anderen beiden Konstruktionen hergestellt werden kann, sei noch einmal eine für die reflexive Partikelverbkonstruktion charakteristische Eigenschaft hervorgehoben, die sich unmittelbar auf die Analyse der Koerzionsstufen, wie ich sie in Unterabschnitt 7.4.1 für die reflexive Bewegungskonstruktion dargestellt habe, auswirkt. Dieses Charakteristikum betrifft die Eigenschaft des KE 〈WEG〉 als Nicht-Kern-KE, das fakultativ instanziiert werden kann (Unterabschnitt 6.4.2). Für die Klassifikation der Konstrukte der reflexiven Partikelverbkonstruktion in sieben Koerzionsstufen stellt die Eigenschaft des KE 〈WEG〉 als Nicht-Kern-KE eine Herausforderung dar. Der Unterschied zwischen seiner Instanziierung und seiner Nicht-Instanziierung sei zunächst an einigen Beispielen illustriert.

Die beiden Konstrukte in (48) und (49) sind Beispiele für den lexikalischen Frame Manipulation, wobei das KE 〈WEG〉 in (48) instanziiert ist und in (49) uninstanziiert bleibt.

(48) \{[Bewegendes Er] [EReignis zwängte] [KEE sich] [〈WEG $\rangle$ zwischen Zug und Tunnelwand] [Richtung hindurch]\}. (Glavinic, Thomas: Die Arbeit der Nacht, München Wien: Carl Hanser Verlag 2006, S. 364)

(49) $\{$ [Bewegendes Wer] [KEE sich] da schnell mit [Richtung rein][EReignis quetschte]\}, hatte gleich sein Pils und seinen Köm auf dem Tisch. (Die Zeit, 09.03.2000, Nr. 11)

Ein zweites Beispielpaar sei mit den Konstrukten in (50) und (51) für den lexikalischen Frame Impact herangezogen, wobei auch hier das KE 〈WEG〉 in (50) instanziiert ist und in (51) uninstanziiert bleibt.

(50) „Repetieren: morgen, in einer Woche, in einem Monat“ heißt Staubs Rezept, damit $\left\{\right.$ KKEE $_{\text {Sich] [BEwEGENDES }}$ das Reingehämmerte] [ ${ }_{\langle\mathrm{WEG}\rangle}$ bis ins Langzeitgedächtnis] [RIchtung durch][EREIGNis schlägt]\}. (Die Zeit, 16.03.2000, Nr. 12)

(51) Sie sprachen jenes flüssige, großzügig dem Englischen entliehene universale Patois junger Europäer, mit dem \{[KeE sich] [Bewegendes Pardell] selbst meistens [Richtung durch][EREIGNis schlug]\}, wenn er nicht sein rührendes, vor lauter Umständlichkeit manchmal für Momente erstarrendes Französisch ausprobierte. (Kopetzky, Steffen: Grand Tour, Frankfurt am Main: Eichborn 2002, S. 230)

An den jeweils ersten Konstrukten dieser beiden Beispielpaare lässt sich die Koerzionsstufe wie in Unterabschnitt 7.4.1 beschrieben einfach ermitteln, da sie (das 
KEE RICHTUNG ausgeklammert) durch die Instanziierung des KE 〈WEG〉 über dieselbe Anzahl von KtE und KEE verfügen wie Konstrukte der reflexiven Bewegungskonstruktion. ${ }^{37}$ Sieht man sich die semantische Motivierung der Strukturelemente der beiden Konstrukte in (48) und (50) an, so ergibt sich das in (52) und (53) dargestellte Bild.

(52) Er [Manipulation zwängte] [EnTtтy sich $^{\text {THEME}] ~[z w i s c h e n ~ Z u g ~ u n d ~ T u n n e l w a n d ~}$ $\mathrm{PATH}]$ hindurch. (Glavinic, Thomas: Die Arbeit der Nacht, München Wien: Carl Hanser Verlag 2006, S. 364)

(53) „Repetieren: morgen, in einer Woche, in einem Monat“ heißt Staubs Rezept, damit [sich ${ }^{\text {THEME}] ~ d a s ~ R e i n g e h a ̈ m m e r t e ~[b i s ~ i n s ~ L a n g z e i t g e d a ̈ c h t n i s ~}$ GoAL] durch[Impact schlägt]. (Die Zeit, 16.03.2000, Nr. 12)

In (52) ist das KEE doppelt motiviert, während das KtE des KE 〈WEG $\rangle$ einfach durch den Konstruktions-Frame motiviert ist. Indes sind in (53) sowohl das KtE des KE $\langle$ WEG $\rangle$ als auch das KEE sind einfach durch den Konstruktions-Frame motiviert. Somit kommt nach den in Unterabschnitt 7.4.1 festgehaltenen Prinzipien dem Konstrukt in (52) Koerzionsstufe $6 \mathrm{zu}$, während das Konstrukt in (53) Koerzionsstufe 7 zuzuordnen ist.

Bei Konstrukten wie denjenigen in (49) und (51) ist die Festlegung der Koerzionsstufe jedoch nicht ohne Weiteres analog zur reflexiven Bewegungskonstruktion möglich, da kein KtE des KE 〈WEG〉 zur Verfügung steht, dessen semantische Motivierung untersucht werden könnte. Einzig das KEE bleibt hierfür übrig, sodass sich ein Bild wie in (54) und (55) ergibt.

(54) Wer [EnTity sich ${ }^{\text {THEME}] ~ d a ~ s c h n e l l ~ m i t ~ r e i n[M a n i p u l a t i o n ~ q u e t s c h t e], ~ h a t t e ~}$ gleich sein Pils und seinen Köm auf dem Tisch. (Die Zeit, 09.03.2000, Nr. 11)

(55) Sie sprachen jenes flüssige, großzügig dem Englischen entliehene universale Patois junger Europäer, mit dem [sich THEME] Pardell selbst meistens durch[Impact schlug], wenn er nicht sein rührendes, vor lauter Umständlichkeit manchmal für Momente erstarrendes Französisch ausprobierte. (Kopetzky, Steffen: Grand Tour, Frankfurt am Main: Eichborn 2002, S. 230)

37 Das KEE Richtung lasse ich bei der Ermittlung der sieben Koerzionsstufen für die reflexive Partikelverbkonstruktion unberücksichtigt, weil es, wie in Unterabschnitt 6.4.2 gesehen, keine Varianz in seiner semantischen Motivierung aufweist, sondern in der Regel durch das FE Motion.DIRECTION motiviert ist. 
Zwar ist die Koerzionsstufe für Beispiele wie (54) und (55) aufgrund der für denselben lexikalischen Frame belegten Konstrukte in (52) und (53) prinzipiell vorhersagbar, allerdings können, wie in Unterabschnitt 7.4.1 dargestellt, lexikalische Frames nicht pauschal auf bestimmte Koerzionsstufen festgelegt werden, da die Konstitution eines Konstrukt-Frames und somit die semantische Motivierung eines Konstrukts innerhalb desselben lexikalischen Frames variieren kann. Liegt lediglich das KEE zur Bestimmung der Koerzionsstufe vor, wie in Konstrukten der reflexiven Partikelverbkonstruktion ohne instanziiertes $\mathrm{KE}\langle\mathrm{WEG}\rangle$, ist die Koerzionsstufe also schwieriger zu bestimmen.

Hinzu kommt, dass durch die Tatsache, dass das $\mathrm{KE}\langle\mathrm{WEG}\rangle$ der reflexiven Partikelverbkonstruktion ein Nicht-Kern-KE ist, weitere mögliche Varianten von Konstrukt-Frames, nach denen ich die sieben Koerzionsstufen in Unterabschnitt 7.4.1 ausgerichtet habe, berücksichtigt werden müssen. Über die sieben bereits diskutierten Kombinationen der semantischen Motivierung der einzelnen Strukturelemente hinaus kommen nun noch vier für die reflexive Partikelverbkonstruktion spezifische Kombinationen dazu.

1. Das KEE ist einfach durch einen relatierten lexikalischen Frame motiviert, während das KtE des KE 〈WEG〉 uninstanziiert bleibt.

2. Das KEE ist einfach durch einen unrelatierten lexikalischen Frame motiviert, während das KtE des KE 〈WEG〉 uninstanziiert bleibt.

3. Das KEE ist doppelt motiviert, während das KtE des KE 〈WEG〉 uninstanziiert bleibt.

4. Das KEE ist einfach durch den Konstruktions-Frame motiviert, während das KtE des KE 〈WEG〉 uninstanziiert bleibt.

Der erste Fall ist für die reflexive Partikelverbkonstruktion wie bereits für die reflexive Bewegungskonstruktion unproblematisch: Handelt es sich um einen relatierten lexikalischen Frame, beträgt die Koerzionsstufe stets 1. Die anderen drei Fälle sind weniger trivial. Während der zweite Fall für die reflexive Partikelverbkonstruktion nicht belegt ist, exemplifiziert das oben diskutierte Konstrukt in (54) den dritten Fall, das Konstrukt in (55) schließlich fällt unter den vierten Fall. Wie können diese vier Kombinationen der Motivierung und Instanziierung der Strukturelemente von Konstrukten der reflexiven Partikelverbkonstruktion in die Klassifikation von sieben Koerzionsstufen, die ich in Unterabschnitt 7.4.1 vorgestellt habe, eingeordnet werden?

Weil, wie erwähnt, bis auf die erste der vier für die reflexive Partikelverbkonstruktion hinzukommenden Kombinationen von Motivierungen der Strukturelemente ihrer Konstrukte eine Zuordnung allein aufgrund des lexikalischen Frames nicht pauschal möglich ist, möchte ich dafür argumentieren, die vier Fälle jeweils der höchsten Koerzionsstufe zuzuordnen, die aufgrund der vorhandenen Moti- 
vierung des KEE möglich ist. Während also dem ersten Fall Koerzionsstufe 1 zukommt, muss der zweite Fall Koerzionsstufe 3 zugeordnet werden, da diese die höchste Koerzionsstufe mit einer einfachen Motivierung des KEE darstellt. Der dritte Fall erhält Koerzionsstufe 6, weil diese die höchste Koerzionsstufe mit einer doppelten Motivierung des KEE darstellt. Der vierte Fall muss schließlich der höchsten Koerzionsstufe 7 zugeordnet werden, da diese gleichzeitig die höchste Koerzionsstufe mit einer einfachen Motivierung des KEE durch den KonstruktionsFrame darstellt. Die beiden oben diskutierten Konstrukte in (54) und (55) sind also - in dieser Reihenfolge - den Koerzionsstufen 6 und 7 zuzuordnen.

Die Einordnung der vier zusätzlichen Kombinationen von Motivierungen in die sieben Koerzionsstufen ist in Abbildung 7.2 dargestellt, die als eine Erweiterung des in Unterabschnitt 7.4.1 in Abbildung 7.1 für die reflexive Bewegungskonstruktion dargestellten Schemas gelten kann.

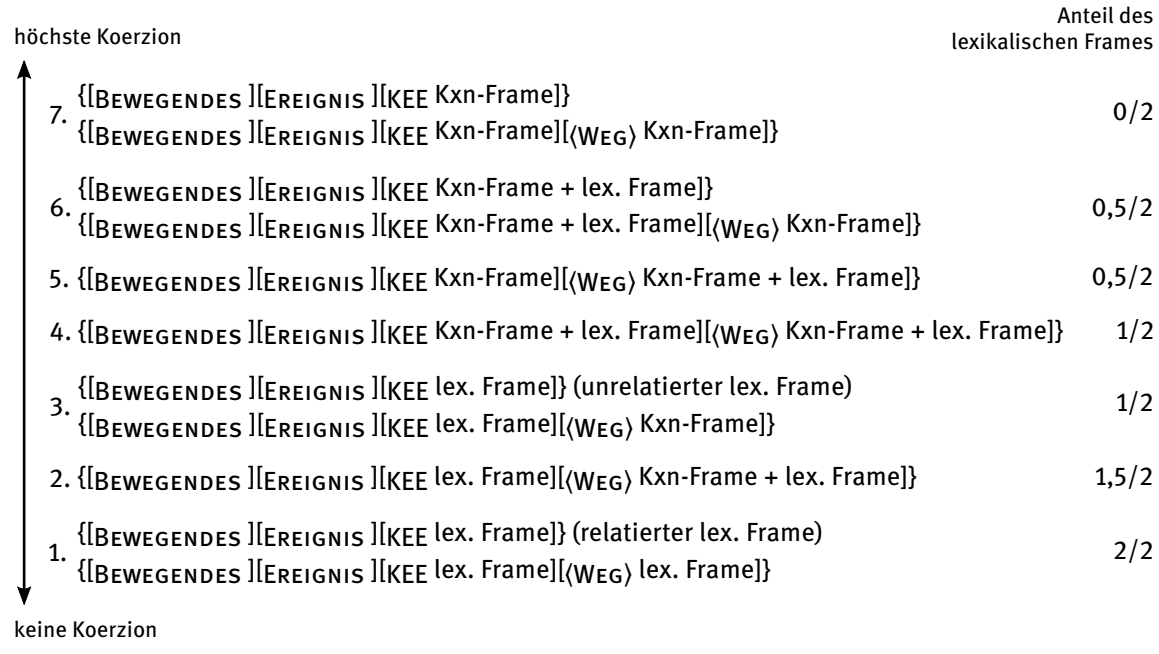

Abb. 7.2: Sieben Koerzionsstufen der reflexiven Partikelverbkonstruktion

Mit dieser für die reflexive Partikelverbkonstruktion erweiterten Aufstellung der sieben Koerzionsstufen kann nun die Verteilung der einschlägigen Konstrukte auf diese ermittelt werden. Diese ist in Tabelle 7.16 zusammengefasst. Die bereits für die reflexive Bewegungskonstruktion unterschiedene Staffelung nach dem Anteil des lexikalischen Frames am Konstrukt-Frame kann für die reflexive Partikelverbkonstruktion auch unter Berücksichtigung der Konstrukte mit uninstanziiertem $\mathrm{KE}\langle\mathrm{WEG}\rangle$ beibehalten werden. 
Tab. 7.16: Koerzionspotenzial der reflexiven Partikelverbkonstruktion

\begin{tabular}{lrr}
\hline Koerzionsstufe & Konstrukte & Anteil \\
\hline 1 & 718 & $84,47 \%$ \\
2 & 0 & \\
3 & 0 & \\
4 & 9 & $1,06 \%$ \\
5 & 25 & $2,94 \%$ \\
6 & 21 & $2,47 \%$ \\
7 & 77 & $9,06 \%$ \\
\hline Gesamt & 850 & $100,00 \%$ \\
\hline
\end{tabular}

Da nun die Verteilung der einzelnen Koerzionsstufen für die reflexive Partikelverbkonstruktion ermittelt wurde, ist es möglich, diese mit derjenigen der reflexiven Bewegungskonstruktion (und beide mit der reflexiven Weg-Konstruktion, vgl. Unterabschnitt 7.4.4) zu vergleichen. Damit ist eine Rangordnung der drei Konstruktionen hinsichtlich ihres Koerzionspotenzials möglich, die letztlich in ihre Konstruktionseinträge eingehen muss.

Der Vergleich zwischen reflexiver Bewegungskonstruktion und reflexiver Partikelverbkonstruktion zeigt zunächst eine Parallele: Die Mehrheit der Konstrukte beider Konstruktionen gehört zu Koerzionsstufe 1. Hierin zeigt sich zunächst direkt der Einflussfaktor der Frame-Nähe, denn der Großteil der Konstrukte beider Konstruktionen besteht aus solchen mit relatierten lexikalischen Frames. Diese hohe Token-Frequenz relatierter lexikalischer Frames deutet zudem auf die Produktivität der beiden Konstruktionen hin, wenngleich jene, wie in Abschnitt 5.6 in Anlehnung an den Produktivitätsbegriff von Barðdal (2008) argumentiert, vorrangig über die Type-Frequenz (relatierter wie unrelatierter) lexikalischer Frames zu bestimmen ist. Ein Zusammenhang dieser drei semantischen Parameter von Konstruktionen (Frame-Nähe, Koerzionspotenzial und Produktivität) spiegelt sich also in den Ergebnissen ihrer Messung wider.

Eine weitere Parallele zwischen reflexiver Bewegungskonstruktion und reflexiver Partikelverbkonstruktion liegt bei den Koerzionsstufen 2 und 3 vor. Bei beiden Konstruktionen kommen diesen beiden Koerzionsstufen die wenigsten Konstrukte zu, wobei Koerzionsstufe 2 stets quantitativ unter Koerzionsstufe 3 liegt. Bei der reflexiven Partikelverbkonstruktion sind, wie Tabelle 7.16 zeigt, für Koerzionsstufe 2 keine Konstrukte belegt. Die Verteilung der Konstrukte beider Konstruktionen verläuft für die ersten drei Koerzionsstufen also nahezu parallel. Interessanter ist nun, wie sich die vier übrigen Koerzionsstufen 4 bis 7 ausdifferenzieren. Dabei erscheint es angebracht, bei der höchsten Koerzionsstufe 7 zu beginnen, denn für beide Konstruktionen fällt auf, dass auf diese Koerzionsstufe 
die zweitmeisten Konstrukte entfallen, sodass sich neben der Parallele in den ersten drei Koerzionsstufen auch hier eine Gemeinsamkeit ergibt. Dies setzt sich auf Koerzionsstufe 5 fort, denn für beide Konstruktionen entfallen auf sie jeweils die drittmeisten Konstrukte. Ein Unterschied ist lediglich in den Koerzionsstufen 4 und $6 \mathrm{zu}$ erkennen. Während für die reflexive Bewegungskonstruktion die viertmeisten Konstrukte auf Koerzionsstufe 4 entfallen, trifft dies bei der reflexiven Partikelverbkonstruktion auf Koerzionsstufe $6 \mathrm{zu}$. Für die fünftmeisten Konstrukte ist die Situation entsprechend umgekehrt.

Welche Schlussfolgerung lässt sich aus diesen Beobachtungen ziehen? Die Tatsache, dass sich die Konstrukte der reflexiven Partikelverbkonstruktion prozentual, wenn auch nur mit einer leichten Tendenz, auf höhere Koerzionsstufen verteilen, spricht dafür, ihr Koerzionspotenzial höher als dasjenige der reflexiven Bewegungskonstruktion einzustufen. Dies ist das für die konstruktikographische Beschreibung der beiden Konstruktionen einschlägige Ergebnis dieser Generalisierung. Dieser Befund kann zudem mit dem Vergleich der Produktivität beider Konstruktionen in Beziehung gesetzt werden, nicht zuletzt um einen möglichen Zusammenhang zwischen Koerzionspotenzial und Produktivität (vgl. dazu Lauwers \& Willems 2011: 1230; Suttle \& Goldberg 2011: 1238) sichtbar zu machen. Bevor ich in Abschnitt 7.5 darauf zurückkomme, soll noch das Koerzionspotenzial der reflexiven Weg-Konstruktion in den Blick genommen werden.

\subsubsection{Koerzionspotenzial der reflexiven Weg-Konstruktion}

Das Koerzionspotenzial der reflexiven Weg-Konstruktion ist das von allen drei Konstruktionen am einfachsten zu ermittelnde. Dies liegt vorrangig an der geringen Produktivität dieser Konstruktion (vgl. Unterabschnitt 7.5.2). Von der reflexiven Bewegungskonstruktion unterscheidet sich die reflexive Weg-Konstruktion allerdings (neben dem zusätzlichen KorE, das für die Messung des Koerzionspotenzials irrelevant ist) darin, dass ihr KE 〈WEG〉 als Nicht-Kern-KE zu klassifizieren ist (vgl. Unterabschnitt 3.3.2). In dieser Hinsicht ist sie aber mit der reflexiven Partikelverbkonstruktion zu vergleichen, sodass das Koerzionspotenzial der reflexiven Weg-Konstruktion analog zu demjenigen der reflexiven Partikelverbkonstruktion ermittelt werden. Ausschlaggebend ist also auch hier die semantische Motivierung des KE 〈WEG sowie des KEE. Die sieben Koerzionsstufen, die ich in Unterabschnitt 7.4.3 in Abbildung 7.2 dargestellt habe, lassen sich direkt auf die reflexive Weg-Konstruktion anwenden. Tabelle 7.17 zeigt die Verteilung der Konstrukte der reflexiven Weg-Konstruktion auf diese sieben Koerzionsstufen.

Von den insgesamt 27 Konstrukten der reflexiven Weg-Konstruktion ist nur ein einziges mit einem unrelatierten lexikalischen Frame belegt. Dieses bereits 
Tab. 7.17: Koerzionspotenzial der reflexiven Weg-Konstruktion

\begin{tabular}{lrr}
\hline Koerzionsstufe & Konstrukte & Anteil \\
\hline 1 & 26 & $96,30 \%$ \\
2 & 0 & \\
3 & 0 & \\
4 & 0 & \\
5 & 1 & $3,70 \%$ \\
6 & 0 & \\
7 & 0 & \\
\hline Gesamt & 27 & $100,00 \%$ \\
\hline
\end{tabular}

mehrfach zitierte Konstrukt, das ich hier in (56) wiederhole, ist Koerzionsstufe 5 zuzuordnen, da das KEE einfach durch den Konstruktions-Frame motiviert wird, während das KtE des KE 〈WEG〉 doppelt motiviert wird.

(56) Mein Schälmesser mit der dünnen Klinge [cutting säbelt] [sich ${ }^{\text {THEME}] ~ e i n e n ~}$ Weg [Iтем durch die buschigen Petersilienköpfe ${ }^{\text {PATH }}$, während ich überlege, ob es tatsächlich Köpfe oder doch Blätter oder gar Büschel heißt. (Riedel, Susanne: Eine Frau aus Amerika, Berlin: Berlin Verlag 2003, S. 106)

Die in Tabelle 7.17 dargestellten Ergebnisse sind vor dem Hintergrund der Daten für die reflexive Bewegungskonstruktion (Tabelle 7.4.2 in Unterabschnitt 7.4.2) und der reflexiven Partikelverbkonstruktion (Tabelle 7.17 in Unterabschnitt 7.4.3) unschwer dahingehend zu interpretieren, dass die reflexive Weg-Konstruktion das geringste Koerzionspotenzial der drei Konstruktionen besitzt. Im Vergleich ist demnach das Koerzionspotenzial der reflexiven Partikelverbkonstruktion am höchsten, dasjenige der reflexiven Bewegungskonstruktion geringfügig niedriger, während dasjenige der reflexiven Weg-Konstruktion wiederum mit deutlichem Abstand am niedrigsten ist. Auch dieser Befund ist mit den Ergebnissen über die Produktivität der drei Konstruktionen zu vergleichen.

\subsection{Produktivität als Teil eines Konstruktionseintrag}

Nach dem Koerzionspotenzial ist die Produktivität der zweite semantische Parameter von Konstruktionen, der, gemäß der Aufstellung in Unterabschnitt 7.1.3, als eigener Datenpunkt in einen Konstruktionseintrag eingehen muss. Dazu muss, ähnlich wie beim Koerzionspotenzial, die Produktivität der zu beschreibenden Konstruktionen zunächst ermittelt werden. Eine Methode dafür möchte ich in 
diesem Abschnitt vorstellen. Die Grundlagen dafür habe ich bereits in Abschnitt 5.6 mit der konstruktionssemantischen Operationalisierung des Produktivitätsbegriffs von Barðdal (2008) gelegt. In diesem Abschnitt soll es deshalb um die Anwendung der dort diskutierten Prinzipien gehen.

In Unterabschnitt 7.5.1 stelle ich das Verfahren zur Messung der Produktivität vor, indem ich noch einmal die beiden Arten lexikalischer Frames, relatierte und unrelatierte (Unterabschnitt 5.4.2) in den Blick nehme und dahingehend rekapituliere, dass ihre jeweiligen Type-Frequenzen als Maßstab für die semantische Kohärenz der Konstrukte einer Konstruktion einerseits (Unterabschnitt 5.6.1) und die Erweiterbarkeit dieser Konstruktion andererseits (Unterabschnitt 5.6.2) dienen können, wie es im Produktivitätsbegriff von Barðdal (2008) angelegt ist. In Unterabschnitt 7.5.2 schließlich stelle ich die Ergebnisse in Form eines Vergleichs der drei untersuchten Konstruktionen vor, um die Höhe ihrer jeweiligen Produktivität empirisch zu begründen.

\subsubsection{Messung der Produktivität}

Wie in Abschnitt 5.6 dargestellt, beruht die Produktivität einer Konstruktion unter Rückgriff auf den Produktivitätsbegriff von Barðdal (2008) auf zwei wesentlichen Faktoren: der semantischen Kohärenz lexikalischer Frames sowie der ,Erweiterbarkeit' der Konstruktion um neue Konstrukte. Dazu habe ich festgehalten, dass die Menge semantisch kohärenter lexikalischer Frames mit derjenigen der relatierten Frames zu identifizieren ist (vgl. Unterabschnitt 5.6.1) sowie dass all diejenigen Konstrukte zur Erweiterbarkeit einer Konstruktion zählen, in deren KonstruktFrames unrelatierte lexikalische Frames eingehen (vgl. Unterabschnitt 5.6.2).

Für die Messung der Produktivität muss nun also auf diese zwei Arten lexikalischer Frames, die ich in Unterabschnitt 5.4.2 unterschieden habe, zurückgegriffen werden: relatierte und unrelatierte. Zur Wiederholung: Relatierte lexikalische Frames sind all diejenigen, die entweder (i) direkt dem Konstruktions-Frame entsprechen oder (ii) zum Konstruktions-Frame in einer (positiven oder negativen) FrameNähe stehen. Eine Erweiterung der Konstruktion um einen ,neuen` lexikalischen Frame liegt dann vor, wenn der lexikalische Frame nicht zum Spektrum dieser relatierten lexikalischen Frames gehört. Kurzum: ,Neue‘ lexikalische Frames sind alle unrelatierten lexikalischen Frames, die für eine Konstruktion belegt sind. Bezogen auf die Konstrukte einer Konstruktion lassen sich also anhand dieser zwei Arten von lexikalischen Frames zwei Arten von Konstrukten differenzieren, auf die ich bereits in Unterabschnitt 5.4.2 hingewiesen habe:

a) Konstrukte mit relatierten lexikalischen Frames, in denen der lexikalische Frame dem Konstruktions-Frame entspricht oder in Frame-Nähe zu diesem steht; 
b) Konstrukte mit unrelatierten lexikalischen Frames, in denen der lexikalische Frame nicht in Frame-Nähe zum Konstruktions-Frame steht, aber gemeinsam mit diesem den Konstrukt-Frame konstituiert.

Die Summe dieser Belege bildet, wie bereits mehrfach erwähnt, gleichzeitig alle relevanten Konstrukte der Konstruktion ab. Belege, die zwar strukturell den Konstrukten einer Konstruktion wie der reflexiven Bewegungskonstruktion entsprechen, deren lexikalischer Frame aber keinem dieser beiden Kriterien entspricht, sind nicht als Konstrukte der betreffenden Konstruktion einzustufen (vgl. aber Unterabschnitt 6.4.3 für eine Ausnahme bei der reflexiven Partikelverbkonstruktion). Es handelt sich hier um einen Fall von konstruktioneller Ambiguität: unterschiedlicher semantischer Eigenschaften bei gleicher Form (vgl. Goldberg 2002: 335). ${ }^{38}$ Am Beispiel der reflexiven Bewegungskonstruktion können damit Belege wie die in (57)-(59) für die Messung der Produktivität der Konstruktion ausgeschlossen und zugleich als Falschpositive disqualifiziert werden, da sie keine Konstrukte der Konstruktion zeigen. ${ }^{39}$

(57) Martina strich sich durch das Haar, wie früher, aber mit einer älteren Hand. (Kuckart, Judith: Lenas Liebe, Köln: DuMont Literatur und Kunst Verlag 2002, S. 217)

(58) Diese Furcht verwandelt sich mehr und mehr in ein Vorurteil, um nicht zu sagen, in eine Ausrede. (Die Zeit, 05.01.2000, Nr. 2)

(59) Der Trend zur Spezialisierung ergibt sich vor allem aus dem zunehmend härteren Wettbewerb. (Die Zeit, 30.03.2000, Nr. 14)

Die Frage nach dem Vorliegen eines unrelatierten lexikalischen Frames und damit der Einstufung einer Instanz als Konstrukt einer gegebenen Konstruktion lässt sich über die Betrachtung der Strukturparallelen zwischen den (potenziellen) KtE und den FE des lexikalischen Frames und des Konstruktions-Frames beantworten, die ich in Kapitel 6 ausführlich analysiert habe. Die dort erzielten Ergebnisse sind eine wichtige Voraussetzung zur Messung der Produktivität einer Konstruktion, ebenso wie bereits für das Koerzionspotenzial. Wie aber kann diese konkret durchgeführt werden?

38 Konstruktionelle Ambiguität ist nicht zu verwechseln mit konstruktioneller Polysemie, da für Letztere gilt: „[T]he same form is paired with different but related senses.“ (Goldberg 1992: 51). Eine Verbindung zweier oder mehrerer Interpretationen liegt bei konstruktioneller Ambiguität nicht vor (vgl. Goldberg 2009: 208, Anm. 6). Auf die Relevanz von konstruktioneller Ambiguität komme ich in Unterabschnitt 8.4.2 zurück.

39 Zur Herkunft der Daten vgl. Unterabschnitt 3.4.2. 
Während sich in der Unterscheidung zwischen relatierten und unrelatierten lexikalischen Frames das Kriterium der semantischen Kohärenz widerspiegelt (dadurch, dass alle Konstrukte mit relatierten lexikalischen Frames semantisch kohärent sind und alle mit unrelatierten nicht, vgl. Unterabschnitt 5.6.1), kommt nun das von Barðdal (2008: 27) in ihrem Produktivitätsbegriff verankerte Kriterium der Type-Frequenz hinzu. Zur Messung der Produktivität gilt es somit, für beide Mengen von Frames, relatierte und unrelatierte, deren jeweilige Type-Frequenz zu ermitteln. Als Type-Frequenz ist hier schlicht die Anzahl unterschiedlicher lexikalischer Frames zu verstehen, die für die beiden Mengen belegt sind. Wie ich bereits in Unterabschnitt 5.6.2 argumentiert habe, kommt dabei der Type-Frequenz unrelatierter lexikalischer Frames eine besondere Rolle zu, weil sie diejenigen Konstrukte repräsentiert, die Ausdruck der Erweiterbarkeit der Konstruktion sind. Zur Messung der Produktivität einer Konstruktion sind somit diejenigen Konstrukte, in denen Koerzionseffekte stattfinden, besonders interessant, da sie, wie soeben für die Messung des Koerzionspotenzials gezeigt (Unterabschnitt 7.4.1) mit derjenigen Anzahl der Konstrukte, in deren Konstrukt-Frames unrelatierte lexikalische Frames eingehen, zu identifizieren sind. Der Schluss, dass gerade sie und weniger die Konstrukte mit relatierten lexikalischen Frames ein Indikator für die Produktivität der Konstruktion sind, liegt damit nahe. Um das Verhältnis zwischen Konstrukten mit relatierten und Konstrukten mit unrelatierten lexikalischen Frames zu bestimmen und somit die Produktivität der Konstruktion im Hinblick auf die Konstitution ,neuer‘ Konstrukte zu messen, müssen beide Mengen von Konstrukten in ihrer Type-Frequenz verglichen werden. ${ }^{40}$ Zugespitzt könnte man den Zusammenhang so formulieren: Je höher die Type-Frequenz der unrelatierten lexikalischen Frames im Vergleich zu derjenigen der relatierten lexikalischen Frames, desto höher die Produktivität der Konstruktion. Im folgenden Unterabschnitt 7.5.2 zeige ich anhand eines Vergleichs der Produktivität der drei untersuchten Konstruktionen, wie diese Zusammenhänge operationalisiert werden können.

\subsubsection{Vergleich der untersuchten Konstruktionen}

Grundlage des Vergleichs der drei untersuchten Konstruktionen hinsichtlich ihrer Produktivität sind die für sie jeweils belegten lexikalischen Frames, die danach geordnet werden können, ob sie relatiert oder unrelatiert sind. Für jede Konstruk-

40 Die Frage, wie hoch die Type-Frequenz einer Konstruktion allgemein sein muss, um von einer gewissen Produktivität zu sprechen, ist eine offene Frage, auf die bereits Croft (2007: 504) hinweist. Klar ist aber in jedem Fall, dass nur ein Vergleich unterschiedlicher Konstruktionen Basis für eine solche Einschätzung sein kann. 
tion lässt sich nun die Anzahl der Types, also der unterschiedlichen lexikalischen Frames, ermitteln, die für diese Arten von lexikalischen Frames belegt sind.

Tabelle 7.18 zeigt die Gegenüberstellung von relatierten und unrelatierten lexikalischen Frames für die reflexive Bewegungskonstruktion. Von allen möglichen relatierten lexikalischen Frames (vgl. die Tabellen 5.12 bis 5.17 in Unterabschnitt 5.4.3), sind 16 belegt. Somit liegt die Anzahl der Types relatierter lexikalischer Frames bei ebendiesem Wert. Demgegenüber sind 34 unterschiedliche unrelatierte lexikalische Frames belegt, die den Type-Wert dieser Art lexikalischer Frames bilden. Von allen für die reflexive Bewegungskonstruktion belegten Konstrukten beträgt der Anteil der ,neuen“ Konstrukte somit 68,00 \% und liegt damit auf Basis der Type-Frequenzen deutlich über dem Anteil der relatierten lexikalischen Frames, der 32,00 \% beträgt. Die Produktivität der reflexiven Bewegungskonstruktion kann damit als hoch eingestuft werden, insbesondere wenn man sie mit den anderen beiden untersuchten Konstruktionen vergleicht. ${ }^{41}$

Tab. 7.18: Produktivität der reflexiven Bewegungskonstruktion

\begin{tabular}{lrr}
\hline Lexikalische Frames & Types & Anteil \\
\hline Relatiert & 16 & $32,00 \%$ \\
Unrelatiert & 34 & $68,00 \%$ \\
\hline Gesamt & 50 & $100,00 \%$ \\
\hline
\end{tabular}

Zwar ist die Produktivität der reflexiven Bewegungskonstruktion aufgrund dieser Befunde grundsätzlich als hoch einzustufen, noch höher aber liegt die Produktivität der reflexiven Partikelverbkonstruktion. Wie Tabelle 7.19 zeigt, liegt einerseits die Type-Frequenz der relatierten lexikalischen Frames unter derjenigen der reflexiven Bewegungskonstruktion, andererseits liegt die Type-Frequenz der unrelatierten lexikalischen Frames über derjenigen der reflexiven Bewegungskonstruktion. Dies wirkt sich auf das Verhältnis von relatierten und unrelatierten lexikalischen Frames aus: So stehen 10 (21,28 \%) relatierte lexikalische Frames $37(78,72 \%)$ unrelatierten lexikalischen Frames gegenüber. Die gesamte TypeFrequenz (47 Frames) ist dabei interessanterweise fast mit derjenigen der reflexiven Bewegungskonstruktion (50 Frames) identisch. Da das Verhältnis zwischen relatierten und unrelatierten lexikalischen Frames bei der reflexiven Partikel-

41 Kunze (1997: 135) spricht der reflexiven Bewegungskonstruktion „höchstens einen mittleren Grad an Produktivität“ zu. Wie er zu dieser Annahme kommt und vor welchem Vergleichshintergrund dies geschieht, erläutert er allerdings nicht. 
Tab. 7.19: Produktivität der reflexiven Partikelverbkonstruktion

\begin{tabular}{lrr}
\hline Lexikalische Frames & Types & Anteil \\
\hline Relatiert & 10 & $21,28 \%$ \\
Unrelatiert & 37 & $78,72 \%$ \\
\hline Gesamt & 47 & $100,00 \%$ \\
\hline
\end{tabular}

verbkonstruktion (1:3,7) aber deutlich höher zugunsten Letzterer ausfällt als bei der reflexiven Bewegungskonstruktion (1:2,13), ist die Produktivität der reflexiven Partikelverbkonstruktion als höher einzustufen. Liegt wie bei der reflexiven Bewegungskonstruktion und der reflexiven Partikelverbkonstruktion eine nahe$\mathrm{zu}$ identische Gesamt-Type-Frequenz relatierter und unrelatierter lexikalischer Frames vor, entscheidet also das Verhältnis zwischen diesen beiden Mengen lexikalischer Frames. Liegt es für eine Konstruktion, wie hier für die reflexive Partikelverbkonstruktion, höher zugunsten unrelatierter lexikalischer Frames, ist diese Konstruktion als produktiver einzustufen.

Die Produktivität der reflexiven Weg-Konstruktion, deren quantitative Grundlage in Tabelle 7.20 zusammengefasst ist, stellt sich schließlich als niedrigste unter allen drei untersuchten Konstruktionen dar. Dies lässt sich bereits an der absoluten Verteilung der lexikalischen Frames ablesen, von denen insgesamt nur zwei Types belegt sind. Neben dem einzigen relatierten lexikalischen Frame Motion, der zugleich den Konstruktions-Frame darstellt und den die LE bahnen evoziert (zur Begründung Unterabschnitt 3.3.2), ist lediglich ein unrelatierter Frame belegt, den die LE säbeln (etwa: cut.v) evoziert, nämlich Cutting. Die vermutete geringe Produktivität der reflexiven Weg-Konstruktion im Vergleich zur reflexiven Bewegungskonstruktion lässt sich also bestätigen. Trotz dieser sehr geringen Produktivität ist für die reflexive Weg-Konstruktion somit eine über die LE bahnen und den relatierten lexikalischen Frame Motion noch hinausgehende Produktivität zu beobachten, was die Annahme von Mortelmans \& Smirnova (2020: 60), ,the construction may be considered a fixed idiomatic expression with a high degree of entrenchment and lexicalization“, fragwürdig erscheinen lässt.

Tab. 7.20: Produktivität der reflexiven Weg-Konstruktion

\begin{tabular}{lrr}
\hline Lexikalische Frames & Types & Anteil \\
\hline Relatiert & 1 & $50,00 \%$ \\
Unrelatiert & 1 & $50,00 \%$ \\
\hline Gesamt & 2 & $100,00 \%$ \\
\hline
\end{tabular}


Ein elaboriertes Bild der Produktivität der drei Konstruktionen lässt sich freilich erst dann ermitteln, wenn man Daten für weitere Argumentstruktur-Konstruktionen erhebt und diese entsprechend vergleicht. Das hier vorgeschlagene Verfahren soll eine Möglichkeit bieten, zukünftige Studien zu ermöglichen, ist aber selbstverständlich zunächst auf ähnlich strukturierte Konstruktionen wie die drei hier untersuchten beschränkt. Die ermittelten Daten können jedoch bereits jetzt für eine konstruktikographische Beschreibung verwendet werden und Eingang in Konstruktionseinträge finden. Es ist hierbei nötig, in jedem Konstruktionseintrag einen Vergleich $\mathrm{zu}$ den beiden verwandten Konstruktionen herzustellen, um die Produktivität einer einzelnen Konstruktion vor dem Hintergrund dieses Vergleichs einordnen zu können.

Abschließend sei darauf hingewiesen, dass die Reihenfolge der drei Konstruktionen hinsichtlich ihrer Produktivität exakt ihrer Reihenfolge hinsichtlich des Koerzionspotenzials entspricht, wie sie in den Unterabschnitten 7.4.2 bis 7.4.4 dokumentiert ist. Ob es sich hierbei um einen Zufall handelt, der speziell für die drei untersuchten Konstruktionen beobachtet werden kann, oder ob dies Anlass zur Annahme einer systematischen Korrelation zwischen diesen beiden semantischen Parametern liefert, kann nur durch größere Datenmengen und letztlich weitere Studien zu anderen Konstruktionen beantwortet werden. ${ }^{42}$

\subsection{Emergente Struktur als Teil eines Konstruktionseintrags}

Der letzte semantische Parameter, der als eigener Datenpunkt in einen Konstruktionseintrag eingehen muss, ist die emergente Struktur. Für die drei untersuchten Konstruktionen betrifft sie den semantischen Aspekt der ,Schwierigkeit', der als Standardwert in einer Konstruktbedeutung enthalten sein kann. In Unterabschnitt 5.7.2 habe ich drei Varianten vorgestellt, die die Evokation dieses Standardwerts durch sprachlich overte Mittel bewirken können. Hinsichtlich der konstruktikographischen Generalisierung dieses semantischen Parameters ist nun zu klären, wie eine geeignete Auswertung der Konstrukte einer betreffenden Konstruktion auf diese drei Varianten hin aussehen und wie sie in einen Konstruktionseintrag eingehen kann. Daneben steht, wie für die beiden anderen semantischen Parameter, die einen eigenen Datenpunkt in einem Konstruktionseintrag bekommen

42 Vgl. aber Lauwers \& Willems (2011: 1230) sowie Suttle \& Goldberg (2011: 1238) für den bisweilen angenommenen Zusammenhang zwischen Produktivität und Koerzion, der bis zu einer Ununterscheidbarkeit beider Phänomene reicht. 
(Abschnitte 7.4 und 7.5), noch der Vergleich der drei untersuchten Konstruktionen aus.

In Unterabschnitt 7.6.1 möchte ich zunächst auf die Frage nach der Messung der emergenten Struktur ausgehend von den drei bereits vorgestellten Varianten ihrer Evokation eingehen. Die Auswertung erfolgt hier weitgehend analog zu derjenigen der konstruktionellen Polysemie (Unterabschnitt 7.2.2) sowie der Messung des Koerzionspotenzials (Unterabschnitt 7.4.1). In Unterabschnitt 7.6.2 soll schließlich der Vergleich der drei untersuchten Konstruktionen auf Basis dieser Auswertung vorgenommen werden.

\subsubsection{Messung der emergenten Struktur}

Ein Vorschlag, die emergente Struktur konstruktionssemantisch zu erfassen, ist, dies habe ich in Unterabschnitt 5.7.1 dargestellt, sie als Standardwert, der in der Konstruktbedeutung eines Konstrukts enthalten ist, zu verstehen. Dieser Standardwert kann als Aspekt der ,Schwierigkeit‘ paraphrasiert werden, mit der sich das Referenzobjekt, auf das das KtE des KE BEWEGENDES referiert, (ggf. metaphorisch) ,bewegt‘ (vgl. dazu Unterabschnitte 3.1.4 und 5.7.2). Zur Messung, wie die Evokation einer solchen emergenten Struktur über die Konstrukte einer Konstruktion verteilt ist, muss also erfasst werden, auf welche Arten ein solcher Standardwert in einer Konstruktbedeutung zustande kommen und ggf. sprachlich overt ausgedrückt werden kann.

Als Annäherung an mögliche Prinzipien, nach denen eine emergente Struktur in einem Konstrukt einer der drei untersuchten Konstruktionen evoziert werden kann, habe ich in Unterabschnitt 5.7.2 am Beispiel der reflexiven Bewegungskonstruktion ${ }^{43}$ drei Varianten diskutiert, die an dieser Stelle kurz wiederholt seien.

a) Der Standardwert ,Schwierigkeit‘ ist bereits in der lexikalischen Bedeutung der LE, mit der das KE EREIGNIS instanziiert wird, angelegt.

b) Der Standardwert ,Schwierigkeit“ entsteht durch einen kollokationalen Zusammenhang zwischen der LE als KtE des KE EREIGNIS und dem KtE des KE WEG bzw. 〈WEG〉.

c) Der Standardwert ,Schwierigkeit‘ wird overt durch die Instanziierung eines (i.d.R. Nicht-Kern-)FE wie z.B. MANNER des lexikalischen Frames oder des Konstruktions-Frames realisiert.

43 Inwieweit die drei bereits in Unterabschnitt 5.7.2 diskutierten Varianten auch für die anderen beiden Konstruktionen, insbesondere die reflexive Partikelverbkonstruktion, angenommen werden können, werde ich im folgenden Unterabschnitt 7.6.2 im Rahmen des Vergleichs der drei Konstruktionen untersuchen. 
Ich verweise im Folgenden auf diese drei Varianten mit den Siglen $a$ bis $c$.

Wie bereits in Unterabschnitt 5.7.2 erwähnt, hat schon Oya (1999: 364) für die reflexive Bewegungskonstruktion darauf hingewiesen, dass nicht jedes Konstrukt der Konstruktion eine emergente Struktur aufweisen muss. Neben den Konstrukten, deren Konstruktbedeutungen einen entsprechenden Standardwert aufweisen, müssen also noch diejenigen Konstrukte betrachtet werden, in denen dies nicht der Fall ist. Diese Konstrukte lassen sich wiederum in zwei Kategorien einteilen:

1. Konstrukte, in deren Konstruktbedeutungen das ,Gegenteil“ des Aspekts einer ,Schwierigkeit‘ enthalten ist, die gewissermaßen also eine ,Einfachheit‘ der ,Bewegung' kodieren.

2. Konstrukte, die gegenüber der emergenten Struktur neutral sind, also deren Konstruktbedeutungen weder den Aspekt der ,Schwierigkeit' der ,Bewegung“ enthalten, noch dessen ,Gegenteil‘.

Sieht man sich nun die Konstrukte der drei untersuchten Konstruktionen an, so lässt sich Punkt 1 als Negation des Ergebnisses der drei Varianten a bis c erfassen. Dabei lässt sich für jede Variante beobachten, dass sie nicht nur in der Lage ist, den Standardwert einer ,Schwierigkeit‘, sondern auch denjenigen einer ,Einfachheit' der ,Bewegung، auszudrücken. Für die Messung des semantischen Parameters der emergenten Struktur muss zu jeder Variante also noch deren Negation hinzukommen, sodass nicht mehr drei, sondern bis hierher nunmehr sechs Varianten erfasst werden müssen. Ich verwende im Folgenden für diese drei negativen Varianten von a bis c die Siglen $\neg a$ bis $\neg c$.

Für alle Konstrukte, die nun nicht unter diese sechs Varianten fallen, muss angenommen werden, dass sie gemäß Punkt 2 über keine emergente Struktur in ihren Konstruktbedeutungen verfügen. Sie werden somit in einer siebten Variante erfasst, die ich im Folgenden schlicht als neutral bezeichne. Die Belege (60)-(62) sind Beispiele für diese Variante.

(60) Die Grenze [Cause_motion schob] sich einfach quer durch Blinjski Kut. (Die Zeit, 27.04.2000, Nr. 18)

(61) Katja [placing legte] sich in dessen Lichtkegel und nahm einige der Zeitungsberichte zum Prozeß vor, die Klein immer von Sarrazin bekam und die sich neben dem Bett stapelten. (Hettche, Thomas: Der Fall Arbogast, Köln: DuMont Buchverlag, 2001, S. 335)

(62) Die Schaffnerin [Body_movement beugt] sich zu den Reisenden hinab, bietet jederlei Dienst und Hilfe an, und man sieht, dass sie die Leute, denen sie dienen will, nicht wahrnimmt. (Die Zeit, 13.01.2000, Nr. 3) 
Mit der Erfassung dieser sieben Varianten der möglichen Evokation der emergenten Struktur, ihres ,Gegenteils‘ oder der diesbezüglichen Neutralität eines Konstrukts kann die Verteilung der Konstrukte einer Konstruktion auf diese sieben Varianten quantitativ untersucht werden. Dies ist die Ausgangslage für einen Vergleich der drei untersuchten Konstruktionen.

\subsubsection{Vergleich der untersuchten Konstruktionen}

Die soeben in Unterabschnitt 7.6.1 vorgestellten sieben Varianten der Evokation einer emergenten Struktur in einem Konstrukt lassen sich hinsichtlich ihrer Verteilung über die Konstrukte der drei untersuchten Konstruktionen analysieren. Dabei ist für jede Konstruktion zunächst die Anzahl der Konstrukte, die jeder einzelnen der sieben Varianten zukommt, zu erfassen. Alle Konstrukte einer Konstruktion lassen sich dadurch auf diese sieben Varianten verteilen.

Für die reflexive Bewegungskonstruktion ergibt sich das in Tabelle 7.21 dargestellte Bild.

Tab. 7.21: Emergente Struktur in den Konstrukten der reflexiven Bewegungskonstruktion

\begin{tabular}{lrr}
\hline Variante & Konstrukte & Anteil \\
\hline a & 184 & $18,20 \%$ \\
$\neg$ a & 3 & $0,30 \%$ \\
b & 60 & $5,93 \%$ \\
$\neg b$ & 3 & $0,30 \%$ \\
$C$ & 45 & $4,45 \%$ \\
$\neg C$ & 21 & $2,08 \%$ \\
neutral & 695 & $68,84 \%$ \\
\hline Gesamt & 1.011 & $100,00 \%$ \\
\hline
\end{tabular}

Für die Auswertung der reflexiven Partikelverbkonstruktion können die sieben vorrangig am Beispiel der reflexiven Bewegungskonstruktion entwickelten Varianten mit einer Einschränkung ebenso beobachtet werden. Diese ergibt sich daraus, dass die Varianten $\mathrm{b}$ und $\neg \mathrm{b}$ aufgrund des Status des KE 〈WEG $\rangle$ als Nicht-KernKE nur für Konstrukte erfasst werden können, in denen dieses KE instanziiert ist. Konstrukte, in denen das KE 〈WEG〉 nicht instanziiert ist, können also nur den Varianten $\neg$ a oder $\neg$ b sowie der neutralen Variante zugeordnet werden. Das entsprechende Ergebnis ist in Tabelle 7.22 zu sehen. 
Tab. 7.22: Emergente Struktur in den Konstrukten der reflexiven Partikelverbkonstruktion

\begin{tabular}{lrr}
\hline Variante & Konstrukte & Anteil \\
\hline a & 166 & $19,53 \%$ \\
$\neg$ a & 4 & $0,47 \%$ \\
b & 17 & $2,00 \%$ \\
$\neg b$ & 1 & $0,12 \%$ \\
$c$ & 11 & $1,29 \%$ \\
$\neg$ c & 1 & $0,12 \%$ \\
neutral & 650 & $76,47 \%$ \\
\hline Gesamt & 850 & $100,00 \%$ \\
\hline
\end{tabular}

Eine ähnliche Situation wie für die reflexive Partikelverbkonstruktion ergibt sich für die reflexive Weg-Konstruktion, da auch ihr KE 〈WEG〉 als Nicht-Kern-KE einzustufen ist. Für sie lassen sich die sieben Varianten der Evokation einer emergenten Struktur aufgrund des Status des KE 〈WEG〉 als Nicht-Kern-KE somit analog zur reflexiven Partikelverbkonstruktion untersuchen. Die Verteilung der nur wenigen Konstrukte der reflexiven Weg-Konstruktion auf die sieben Varianten ist in Tabelle 7.23 dargestellt.

Tab. 7.23: Emergente Struktur in den Konstrukten der reflexiven Weg-Konstruktion

\begin{tabular}{lrr}
\hline Variante & Konstrukte & Anteil \\
\hline $\mathrm{a}$ & 0 & \\
$\neg \mathrm{a}$ & 0 & \\
$\mathrm{~b}$ & 4 & $14,81 \%$ \\
$\neg \mathrm{b}$ & 0 & \\
$\mathrm{c}$ & 5 & $18,52 \%$ \\
$\neg \mathrm{C}$ & 2 & $7,41 \%$ \\
neutral & 16 & $59,26 \%$ \\
\hline Gesamt & 27 & $100,00 \%$ \\
\hline
\end{tabular}

Um die Verteilungen der drei Konstruktionen miteinander zu vergleichen, bietet es sich an, Parallelen und Diskrepanzen hinsichtlich der einzelnen Varianten über die drei Konstruktionen hinweg zu untersuchten. Sieht man sich die Daten der drei Konstruktionen vergleichend an, ist unmittelbar festzustellen, dass die Mehrheit der Konstrukte jeder Konstruktion über keine emergente Struktur in den Konstruktbedeutungen verfügt, also der neutralen Variante zugerechnet werden muss. Dies ist vor dem Hintergrund der Diskussion in Unterabschnitt 5.7.2 erwart- 
bar, da bisherige Forschungen zur way-Konstruktion und der reflexiven Bewegungskonstruktion (jedoch ohne vergleichbare empirische Evidenz) festgestellt haben, dass nicht jedes Konstrukt den semantischen Aspekt der ,Schwierigkeit" enthalten muss (vgl. Oya 1999: 364). Diese Beobachtung bestätigt sich nun nicht nur, sie kann sogar dahingehend konkretisiert werden, dass dies tatsächlich die Mehrheit der Konstrukte der drei untersuchten Konstruktionen betrifft: 68,84 \% für die reflexive Bewegungskonstruktion, $76,47 \%$ für die reflexive Partikelverbkonstruktion und 59,26 \% für die reflexive Weg-Konstruktion.

Interessanter sind nun freilich die Mengen der Konstrukte, in deren Konstruktbedeutungen eine emergente Struktur oder deren ,Gegenteil' evoziert wird, die also den Varianten a, b und c sowie $\neg \mathrm{a}, \neg \mathrm{b}$ und $\neg \mathrm{c}$ zukommen. Im direkten Vergleich zunächst der reflexiven Bewegungskonstruktion und der reflexiven Partikelverbkonstruktion ist zu sehen, dass die Verteilung der Konstrukte der jeweiligen Konstruktionen größtenteils ähnlich verläuft. Betrachtet man zunächst die Varianten $\mathrm{a}$, b und c, diejenigen Konstrukte also, in denen eine emergente Struktur evoziert wird, fällt auf, dass bei beiden Konstruktionen Variante a prozentual die größte Gruppe der Konstrukte mit evozierter emergenter Struktur darstellt. An zweiter Stelle steht bei beiden Konstruktionen Variante b, an dritter Stelle Variante c. Die emergente Struktur wird in Konstrukten dieser beiden Konstruktionen vorrangig also über die lexikalische Bedeutung der LE, die den lexikalischen Frame evoziert, kodiert.

Etwas differenzierter gestaltet sich die Situation bei den Konstrukten der Varianten $\neg \mathrm{a}, \neg \mathrm{b}$ und $\neg \mathrm{c}$. Während sich beide Konstruktionen prozentual bei Variante $\neg$ a und $\neg \mathrm{b}$ ähneln, fällt besonders auf, dass die reflexive Partikelverbkonstruktion über wesentlich weniger Konstrukte der Variante $\neg \mathrm{c}$ verfügt, während für die reflexive Bewegungskonstruktion ein erkennbar größerer Anteil für diese Variante belegt ist. Dies deutet darauf hin, dass die Evokation der emergenten Struktur für die reflexive Bewegungskonstruktion differenzierter verläuft als für die reflexive Partikelverbkonstruktion. Der Status des KE 〈WEG〉 der reflexiven Partikelverbkonstruktion als Nicht-Kern-KE hat damit scheinbar kaum einen Einfluss auf die Evokation der emergenten Struktur nach Variante b, die ja auf dem kollokationalen Zusammenhang dieses KE mit dem KE EREIGNIs beruht. Interessant ist, dass diese eingeschränkte Möglichkeit der Evokation der emergenten Struktur in Konstrukten der reflexiven Partikelverbkonstruktion scheinbar nicht über Variante c, also die Instanziierung eines (i.d.R. Nicht-Kern-)FE wie MANNER, kompensiert wird.

Die größere Differenziertheit der reflexiven Bewegungskonstruktion gilt interessanterweise sowohl für Konstrukte, die eine emergente Struktur evozieren als auch für solche, die den Varianten $\neg \mathrm{a}$, $\neg \mathrm{b}$ und $\neg \mathrm{c}$ angehören, also das ,Gegenteil' einer ,Schwierigkeit' kodieren. Für die konstruktikographische Verarbeitung dieses Parameters kann festgehalten werden, dass die reflexive Bewegungskonstruktion 
der reflexiven Partikelverbkonstruktion in dieser Hinsicht überlegen ist, zumal insgesamt ein größerer Anteil ihrer Konstrukte diesen drei Varianten zuzuordnen ist als bei der reflexiven Partikelverbkonstruktion.

Die wenigen Konstrukte der reflexiven Weg-Konstruktion können die gemeinsamen Tendenzen von reflexiver Bewegungskonstruktion und reflexiver Partikelverbkonstruktion bestätigen. Auch hier ist zu sehen, dass die Mehrheit der Konstrukte gegenüber der emergenten Struktur neutral ist. Ein Unterschied zu den beiden anderen Konstruktionen liegt darin, dass für die reflexive Weg-Konstruktion keine Konstrukte der Variante a belegt sind, dies liegt offenkundig an ihrem sehr niedrigen Koerzionspotenzial (vgl. Unterabschnitt 7.4.4). Die Varianten b und c hingegen sind jeweils mit derselben Anzahl an Konstrukten belegt, eine Verteilung, die derjenigen der anderen beiden Konstruktionen zumindest grob ähnelt. Wenig überraschend ist, dass die Verteilung der emergenten Struktur auf die Konstrukte der reflexiven Weg-Konstruktion im Vergleich aller drei Konstruktionen am wenigsten differenziert ist.

Mit diesem Vergleich der drei untersuchten Konstruktionen lässt sich also wie bereits für das Koerzionspotenzial und die Produktivität eine Reihenfolge hinsichtlich der Verteilung der emergenten Struktur aufstellen. Die reflexive Bewegungskonstruktion kann dabei aus den soeben dargelegten Gründen als am differenziertesten gelten. An zweiter Stelle folgt die reflexive Partikelverbkonstruktion. Diese Reihenfolge stellt sich somit umgekehrt zum jeweiligen Koerzionspotenzial und der Produktivität der beiden Konstruktionen dar. Die reflexive WegKonstruktion liegt, wie bereits hinsichtlich des Koerzionspotenzials und der Produktivität, auf dem dritten Platz hinter den beiden anderen Konstruktionen. 